\title{
Diastereo- and Enantioselective Construction of Hexahydrocoumarin Scaffold via An Organocatalytic Asymmetric [3+3] Cyclization
}

\author{
Xue Yang, Yu-Chen Zhang, Qiu-Ning Zhu, Man-Su Tu* and Feng Shi* \\ School of Chemistry \& Chemical Engineering, and Jiangsu Key Laboratory of Green Synthetic \\ Chemistry for Functional Materials, Jiangsu Normal University, Xuzhou, 221116, China \\ E-mail: mstu2016@126.com; fshi@jsnu.edu.cn
}

\section{Contents:}

1. Screening of catalysts and optimization of reaction conditions (S2-S4)

2. ${ }^{1} \mathrm{H}$ NMR spectra of the control experiments (S5-S6)

3. ${ }^{1} \mathrm{H}$ and ${ }^{13} \mathrm{C}$ NMR spectra of products 3 and 5 (S7-S30)

4. HPLC Traces of products 3 and 5 (S31-S67)

5. X-ray single crystal data for 3ka (S68-S69) 


\section{Screening of catalysts and optimization of reaction conditions}

Table 1. Screening of catalysts and optimization of reaction conditions ${ }^{\mathrm{a}}$

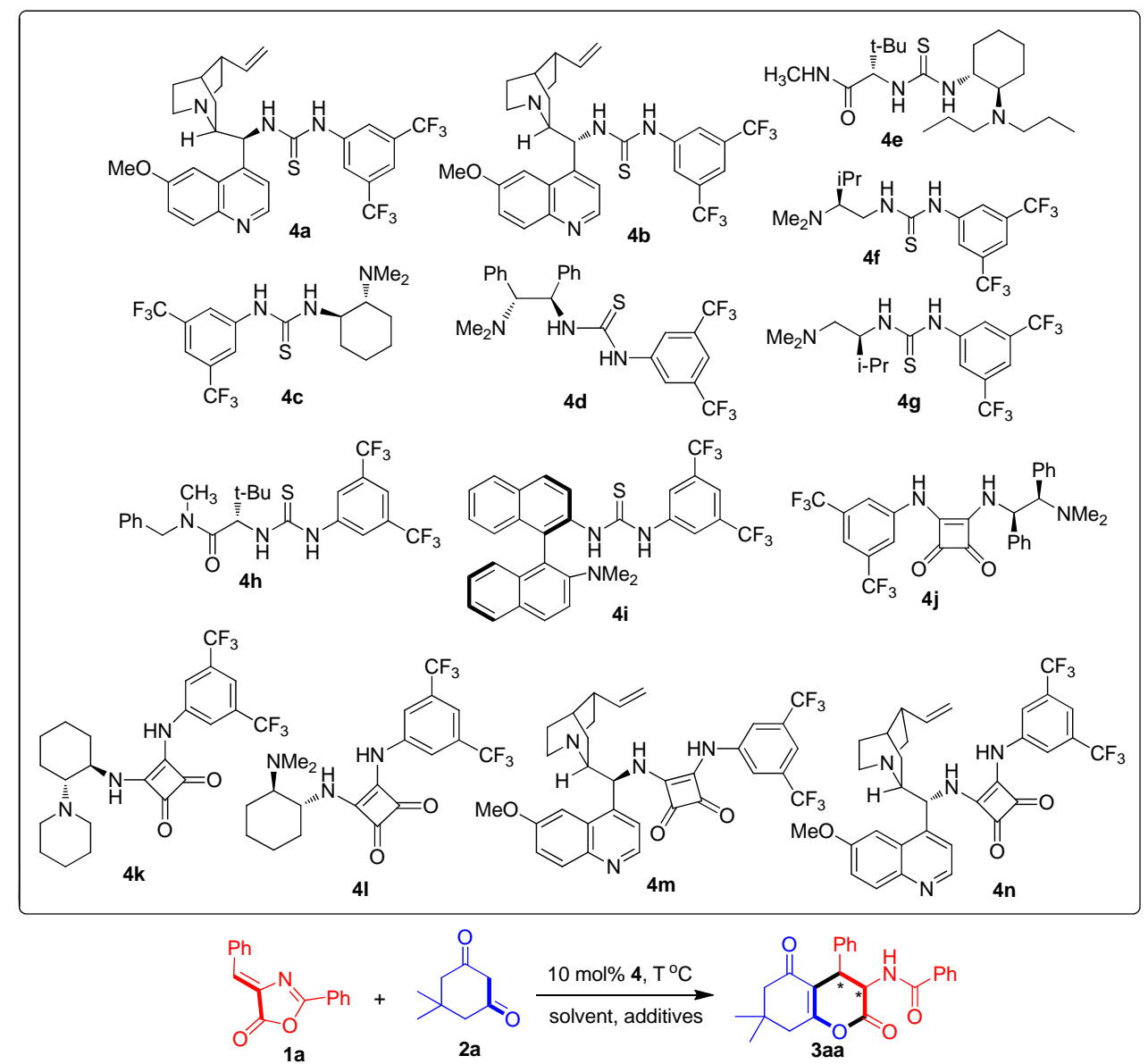

\begin{tabular}{|c|c|c|c|c|c|c|c|c|}
\hline entry & Cat. & solvent & $\mathrm{T}\left({ }^{\circ} \mathrm{C}\right)$ & 1a:2a & additives & yield (\%) & $\mathrm{dr}^{\mathrm{c}}$ & $e^{d}$ \\
\hline 1 & $4 a$ & THF & 50 & 1:1 & $3 \AA \mathrm{MS}$ & 20 & $>95: 5$ & $48: 52$ \\
\hline 2 & $4 b$ & THF & 50 & $1: 1$ & $3 \AA$ MS & 27 & $>95: 5$ & $61: 39$ \\
\hline 3 & $4 c$ & THF & 50 & 1:1 & $3 \AA$ MS & 40 & $>95: 5$ & $68: 32$ \\
\hline 4 & 4d & THF & 50 & 1:1 & $3 \AA \mathrm{MS}$ & 8 & $>95: 5$ & $57: 43$ \\
\hline 5 & $4 e$ & THF & 50 & 1:1 & $3 \AA$ MS & 8 & $>95: 5$ & $58: 42$ \\
\hline 6 & $4 f$ & THF & 50 & 1:1 & $3 \AA$ MS & 13 & $>95: 5$ & $54: 46$ \\
\hline 7 & $4 g$ & THF & 50 & 1:1 & $3 \AA$ MS & 37 & $>95: 5$ & $66: 34$ \\
\hline 8 & $4 h$ & THF & 50 & $1: 1$ & $3 \AA$ MS & trace & $>95: 5$ & - \\
\hline 9 & $4 i$ & THF & 50 & 1:1 & $3 \AA \mathrm{MS}$ & trace & $>95: 5$ & - \\
\hline 10 & $4 j$ & THF & 50 & 1:1 & $3 \AA \mathrm{MS}$ & 12 & $>95: 5$ & $65: 35$ \\
\hline 11 & $4 k$ & THF & 50 & $1: 1$ & $3 \AA$ MS & 12 & $>95: 5$ & $58: 42$ \\
\hline 12 & 41 & THF & 50 & 1:1 & $3 \AA \mathrm{MS}$ & 26 & $>95: 5$ & $55: 45$ \\
\hline 13 & $4 m$ & THF & 50 & 1:1 & $3 \AA \mathrm{MS}$ & 24 & $>95: 5$ & $55: 45$ \\
\hline 14 & $4 n$ & THF & 50 & 1:1 & $3 \AA$ MS & 8 & $>95: 5$ & $60: 40$ \\
\hline 15 & $4 c$ & $\mathrm{CH}_{2} \mathrm{Cl}_{2}$ & 50 & $1: 1$ & $3 \AA$ MS & 80 & $>95: 5$ & $64: 36$ \\
\hline 16 & $4 c$ & AcOEt & 50 & $1: 1$ & $3 \AA$ MS & 77 & $>95: 5$ & $53: 47$ \\
\hline 17 & $4 c$ & toluene & 50 & $1: 1$ & $3 \AA$ MS & 69 & $>95: 5$ & $69: 31$ \\
\hline 18 & $4 c$ & acetone & 50 & 1:1 & $3 \AA \mathrm{MS}$ & 73 & $>95: 5$ & $62: 38$ \\
\hline
\end{tabular}




\begin{tabular}{ccccccccc}
\hline 19 & $\mathbf{4 c}$ & MeCN & 50 & $1: 1$ & $3 \AA$ MS & 55 & $>95: 5$ & $60: 40$ \\
20 & $\mathbf{4 c}$ & toluene & 30 & $1: 1$ & $3 \AA$ MS & 46 & $>95: 5$ & $83: 17$ \\
21 & $\mathbf{4 g}$ & toluene & 30 & $1: 1$ & $3 \AA$ MS & 34 & $>95: 5$ & $89: 11$ \\
22 & $\mathbf{4 g}$ & toluene & 30 & $2: 1$ & $3 \AA$ MS & 53 & $>95: 5$ & $94: 6$ \\
23 & $\mathbf{4 g}$ & toluene & 30 & $3: 1$ & $3 \AA$ MS & 57 & $>95: 5$ & $92: 8$ \\
24 & $\mathbf{4 g}$ & toluene & 30 & $4: 1$ & $3 \AA$ MS & 56 & $>95: 5$ & $93: 7$ \\
25 & $\mathbf{4 g}$ & toluene & 30 & $6: 1$ & $3 \AA$ MS & 61 & $>95: 5$ & $92: 8$ \\
26 & $\mathbf{4 g}$ & toluene & 30 & $1: 2$ & $3 \AA$ MS & 35 & $>95: 5$ & $88: 12$ \\
27 & $\mathbf{4 g}$ & $p$-xylene & 30 & $2: 1$ & $3 \AA$ MS & 59 & $>95: 5$ & $95: 5$ \\
28 & $\mathbf{4 g}$ & $o$-xylene & 30 & $2: 1$ & $3 \AA$ MS & 55 & $>95: 5$ & $94: 6$ \\
29 & $\mathbf{4 g}$ & $m$-xylene & 30 & $2: 1$ & $3 \AA \mathrm{MS}$ & 59 & $>95: 5$ & $96: 4$ \\
30 & $\mathbf{4 g}$ & PhCl & 30 & $2: 1$ & $3 \AA \mathrm{MS}$ & 72 & $>95: 5$ & $83: 17$ \\
31 & $\mathbf{4 g}$ & PhF & 30 & $2: 1$ & $3 \AA \mathrm{MS}$ & 61 & $>95: 5$ & $90: 10$ \\
32 & $\mathbf{4 g}$ & $m$-xylene & 30 & $2: 1$ & $4 \AA \mathrm{MS}$ & 50 & $>95: 5$ & $95: 5$ \\
33 & $\mathbf{4 g}$ & $m$-xylene & 30 & $2: 1$ & $5 \AA \mathrm{MS}$ & 66 & $>95: 5$ & $96: 4$ \\
34 & $\mathbf{4 g}$ & $m$-xylene & 30 & $2: 1$ & - & 13 & $>95: 5$ & $88: 12$ \\
35 & $\mathbf{4 g}$ & $m$-xylene & 10 & $2: 1$ & $5 \AA \mathrm{MS}$ & 11 & $>95: 5$ & $97: 3$ \\
36 & $\mathbf{4 g}$ & $m$-xylene & 0 & $2: 1$ & $5 \AA \mathrm{MS}$ & 8 & $>95: 5$ & $97: 3$ \\
$37^{\text {e }}$ & $\mathbf{4 g}$ & $m$-xylene & 30 & $2: 1$ & $5 \AA \mathrm{MS}$ & 67 & $>95: 5$ & $96: 4$ \\
$38^{\mathrm{e}, \mathrm{f}}$ & $\mathbf{4 g}$ & $m$-xylene & 30 & $2: 1$ & $5 \AA \mathrm{MS}$ & 77 & $>95: 5$ & $96: 4$ \\
\hline
\end{tabular}

${ }^{a}$ Unless otherwise indicated, the reaction was carried out at the $0.1 \mathrm{mmol}$ scale in the presence of $10 \mathrm{~mol} \% 4$ in a solvent (1 mL) with additives (100 mg) for $48 \mathrm{~h} .{ }^{\mathrm{b}}$ Isolated yield. ${ }^{\mathrm{c}} \mathrm{The} d r$ value was determined by ${ }^{1} \mathrm{H}$ NMR and HPLC. ${ }^{\mathrm{d}}$ The $e r$ value was determined by HPLC. ${ }^{\mathrm{e}}$ The reaction was $72 \mathrm{~h} .{ }^{\mathrm{f}} \mathrm{In}$ the presence of $25 \mathrm{~mol} \% \mathbf{4 g}$.

Table 2. Using 1,3-cyclopentanedione $\mathbf{2} \mathbf{c}$ as a substrate in the [3+3] cyclization ${ }^{\mathrm{a}}$

\begin{tabular}{|c|c|c|c|c|}
\hline entry & Cat. & solvent & $\mathrm{T}\left({ }^{\circ} \mathrm{C}\right)$ & yield (\%) \\
\hline 1 & $4 g$ & $m$-xylene & 30 & 0 \\
\hline 2 & $4 g$ & m-xylene & 100 & 0 \\
\hline 3 & DMAP & 1,2-dichloroethane & 80 & 0 \\
\hline 4 & DMAP & THF & 80 & 0 \\
\hline 5 & DMAP & AcOEt & 80 & 0 \\
\hline 6 & DMAP & acetone & 80 & 0 \\
\hline 7 & DMAP & $\mathrm{MeCN}$ & 80 & 0 \\
\hline 8 & DMAP & DMF & 120 & 0 \\
\hline
\end{tabular}

${ }^{\mathrm{a}}$ Unless indicated otherwise, the reaction was carried out at the $0.1 \mathrm{mmol}$ scale in the presence of $40 \mathrm{~mol} \%$ Cat. In solvent $(1 \mathrm{~mL})$ with $5 \AA$ MS (100 mg) as additives for 72 h, and the molar ratio of 1a:2c was 2:1. DMAP = 4-dimethylaminopyridine. 
Table 3. Using acetylacetone $\mathbf{2 e}$ as a substrate in the [3+3] cyclization ${ }^{\mathrm{a}}$

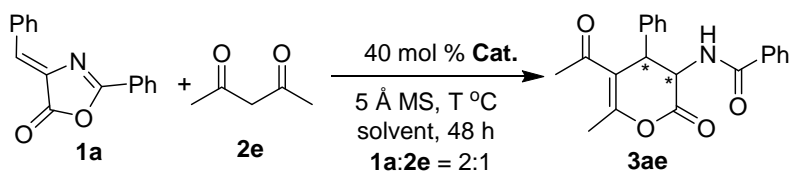

\begin{tabular}{ccccccc}
\hline entry & Cat. & solvent & $\mathrm{T}\left({ }^{\mathrm{o}} \mathrm{C}\right)$ & yield $(\%)^{\mathrm{b}}$ & $\mathrm{dr}^{\mathrm{c}}$ & $\mathrm{er}^{\mathrm{d}}$ \\
\hline 1 & $\mathbf{4 g}$ & $m$-xylene & 30 & 0 & - & - \\
2 & DMAP & 1,2-dichloroethane & 80 & 0 & - & - \\
3 & DMAP & THF & 80 & 0 & - & - \\
4 & DMAP & AcOEt & 80 & 50 & $>95: 5$ & $50: 50$ \\
5 & DMAP & acetone & 80 & 0 & - & - \\
6 & DMAP & MeCN & 80 & 0 & - & - \\
7 & DMAP & m-xylene & 80 & 0 & - & - \\
8 & DMAP & DMF & 100 & 0 & - & - \\
9 & $\mathbf{4 g}$ & AcOEt & 70 & 0 & - & - \\
10 & $\mathbf{4 g}$ & AcOEt & 70 & 0 & - & - \\
11 & $\mathbf{4 c}$ & AcOEt & 70 & 0 & - & - \\
12 & $\mathbf{4 f}$ & AcOEt & 70 & 0 & - & - \\
13 & $\mathbf{4 i}$ & AcOEt & 70 & 0 & - & - \\
14 & $\mathbf{4 j}$ & AcOEt & 70 & 0 & - & - \\
15 & $\mathbf{4 k}$ & AcOEt & 70 & 0 & - & - \\
16 & $\mathbf{4 l}$ & AcOEt & 70 & 0 & - & - \\
17 & $\mathbf{4 m}$ & AcOEt & 70 & 0 & - & - \\
18 & $\mathbf{4 g}$ & m-xylene & 100 & 0 & - & - \\
19 & $\mathbf{4 g}$ & $1,2-$ dichloroethane & 70 & 0 & - & - \\
20 & $\mathbf{4 g}$ & THF & 70 & 32 & $>95: 5$ & $51: 49$ \\
21 & $\mathbf{4 g}$ & acetone & 70 & 0 & - & - \\
22 & $\mathbf{4 g}$ & MeCN & 70 & 0 & - & - \\
\hline
\end{tabular}

${ }^{\mathrm{a}}$ Unless indicated otherwise, the reaction was carried out at the $0.1 \mathrm{mmol}$ scale in the presence of $40 \mathrm{~mol} \%$ Cat. in solvent (1 mL) with $5 \AA$ MS (100 mg) as additives for 48 h, and the molar ratio of 1a:2e was 2:1. ${ }^{\mathrm{b}}$ Isolated yields. ${ }^{\mathrm{c}}$ The $d r$ value was determined by ${ }^{1} \mathrm{H}$ NMR. ${ }^{\mathrm{d}}$ The $e r$ value was determined by HPLC. ${ }^{\mathrm{e}}$ In the presence of 100 mol\% $4 g$. 


\section{2. ${ }^{1} \mathrm{H}$ NMR spectra of the control experiments}

The ${ }^{1} \mathrm{H}$ NMR study on the interaction between chiral catalyst $\mathbf{4} \mathbf{g}$ and substrate $\mathbf{2 a}$ (in DMSO- $d_{6}$ ): Green color: substrate 2a

Blue color: chiral catalyst $\mathbf{4 g}$

Red color: the mixture of chiral catalyst $\mathbf{4 g}$ and substrate $\mathbf{2 a}$
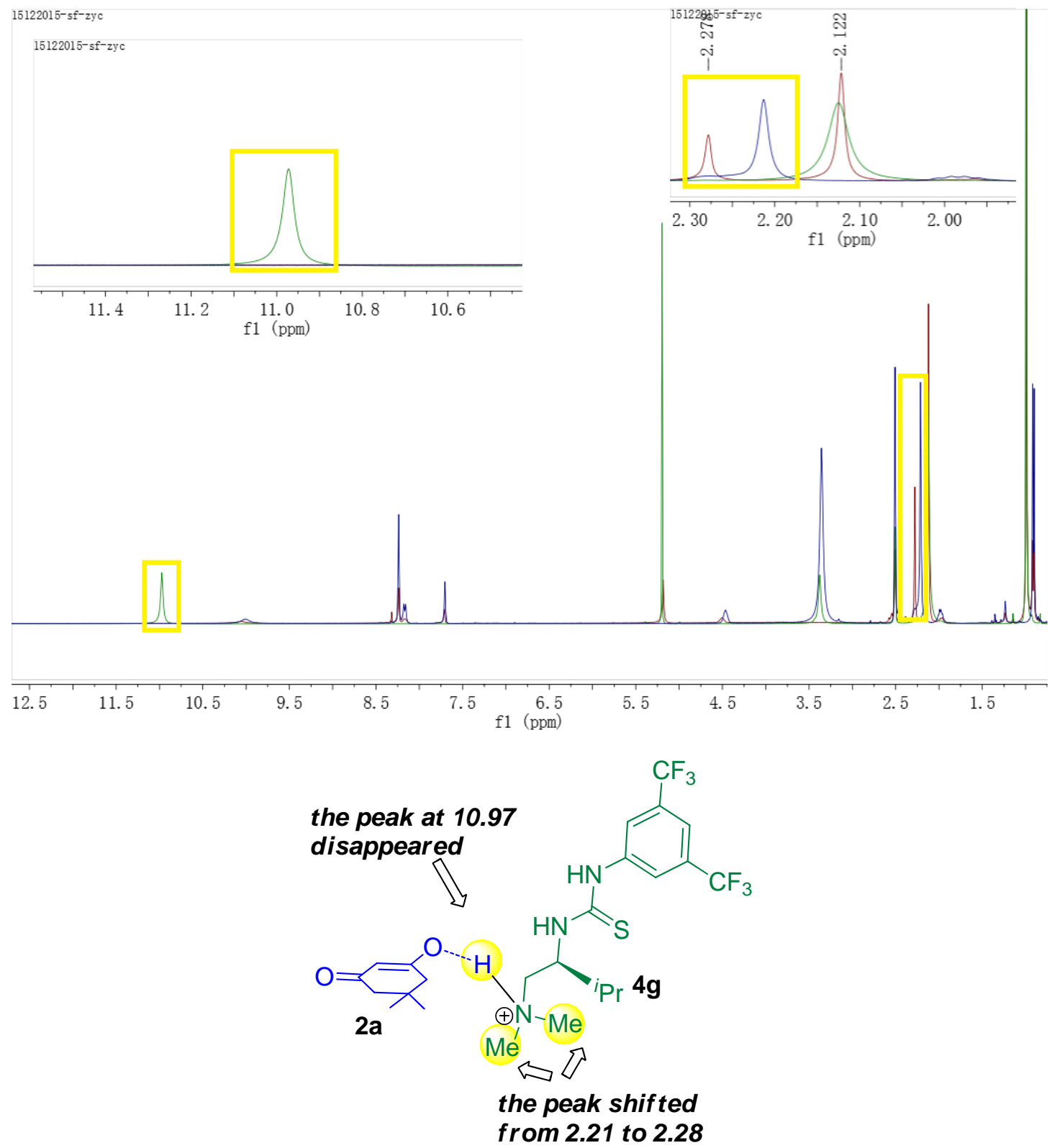
The ${ }^{1} \mathrm{H}$ NMR study on the interaction between chiral catalyst $\mathbf{4 g}$ and substrate $\mathbf{1 a}$ (in $\mathrm{CDCl}_{3}$ ): Green color: substrate 1a

Blue color: chiral catalyst $\mathbf{4 g}$

Red color: the mixture of chiral catalyst $\mathbf{4 g}$ and substrate $\mathbf{1 a}$
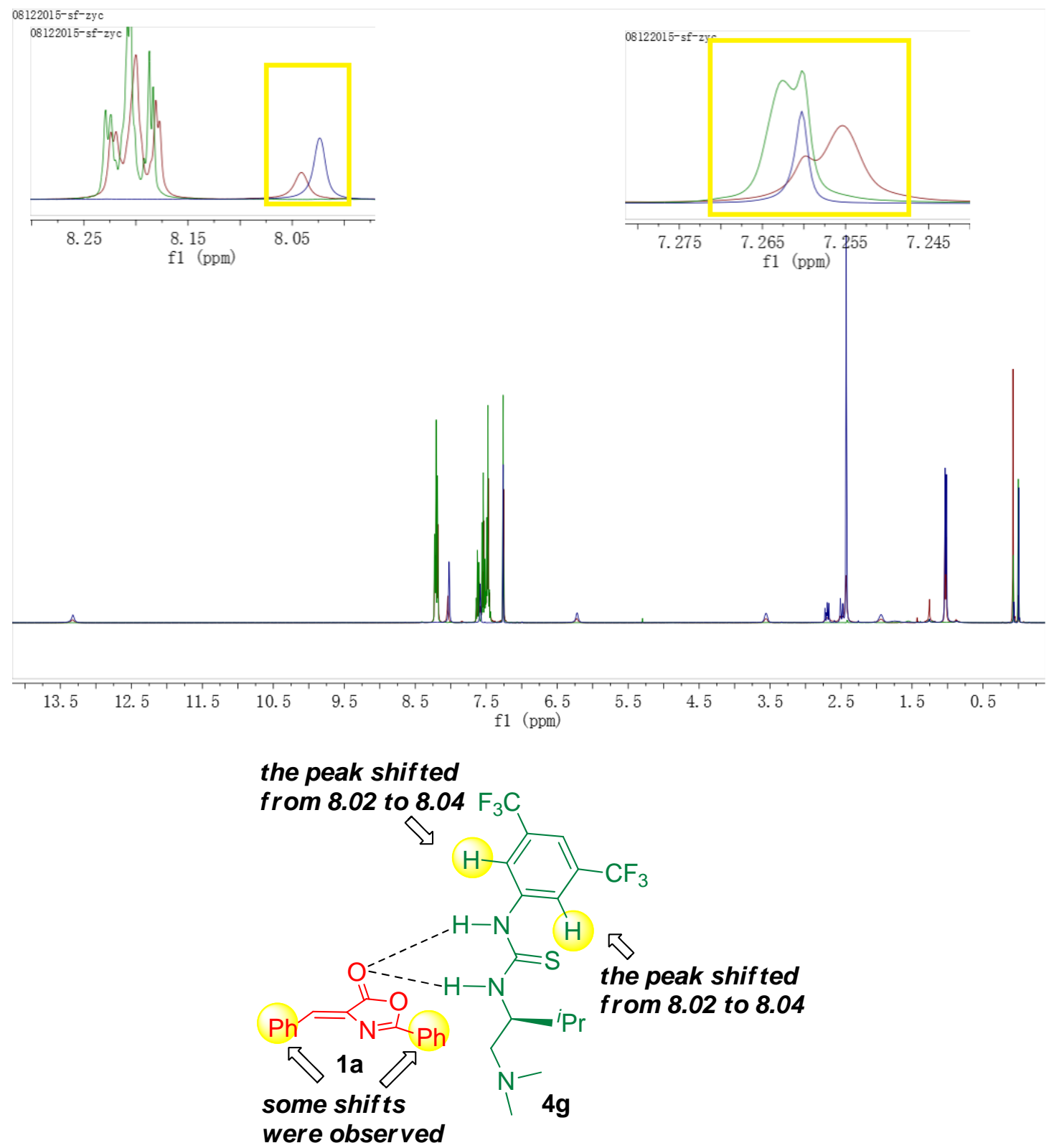


\section{3. ${ }^{1} \mathrm{H}$ and ${ }^{13} \mathrm{C}$ NMR spectra of products 3 and 5}

\section{3aa}
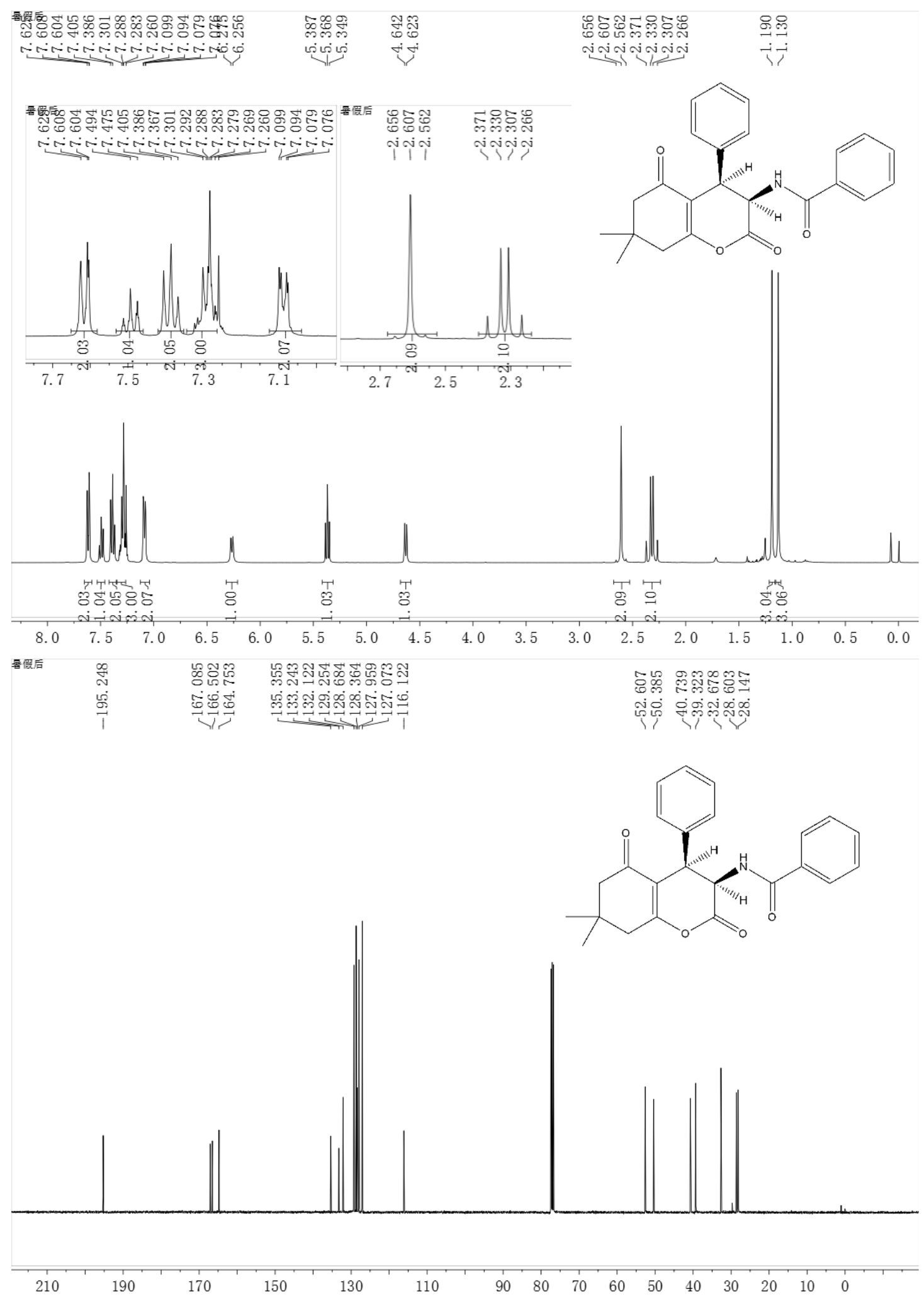


\section{3ba}

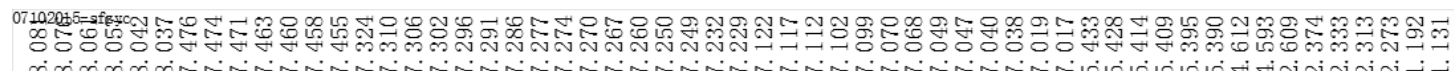

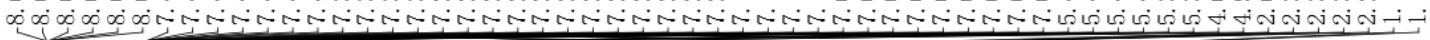
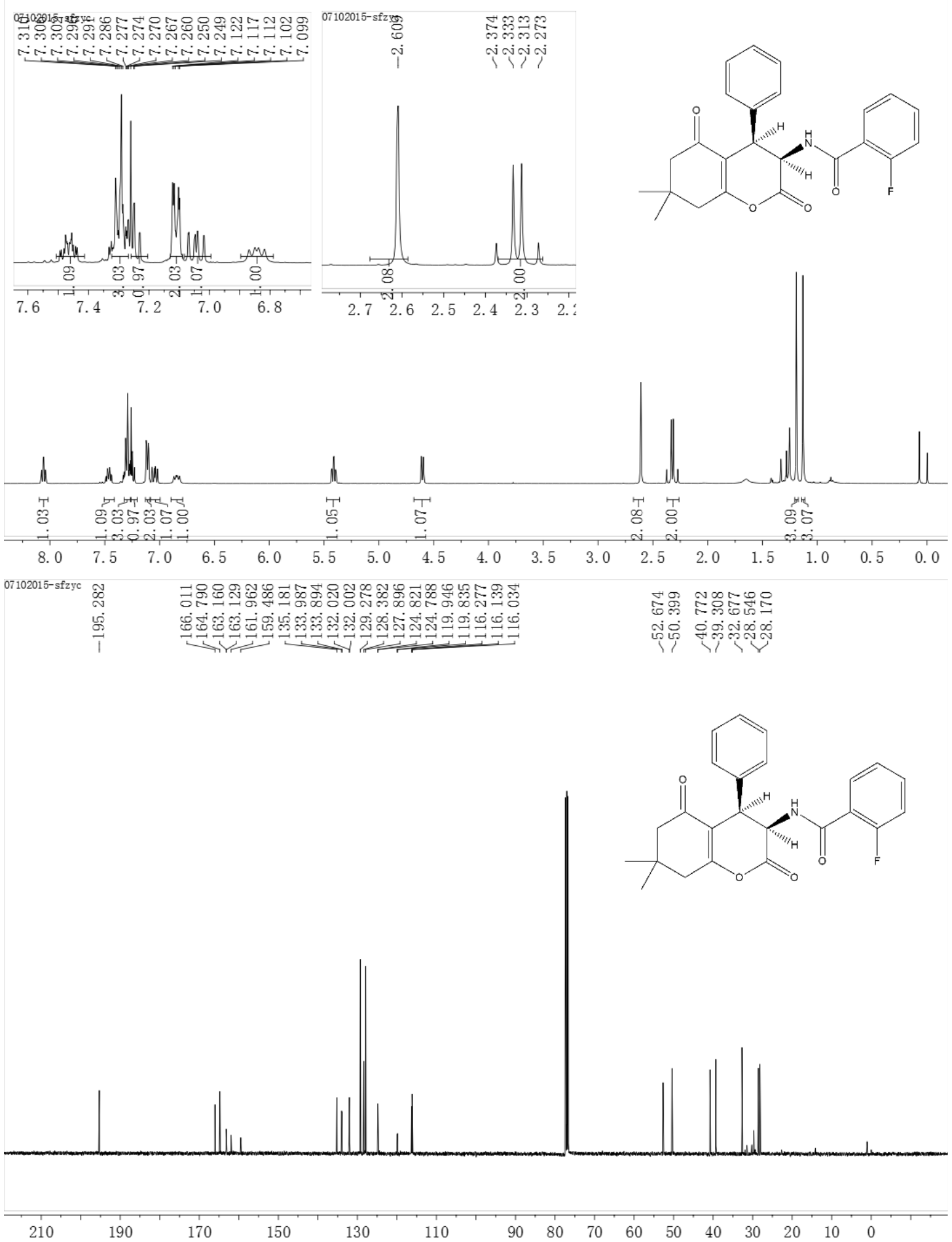


\section{3ca}
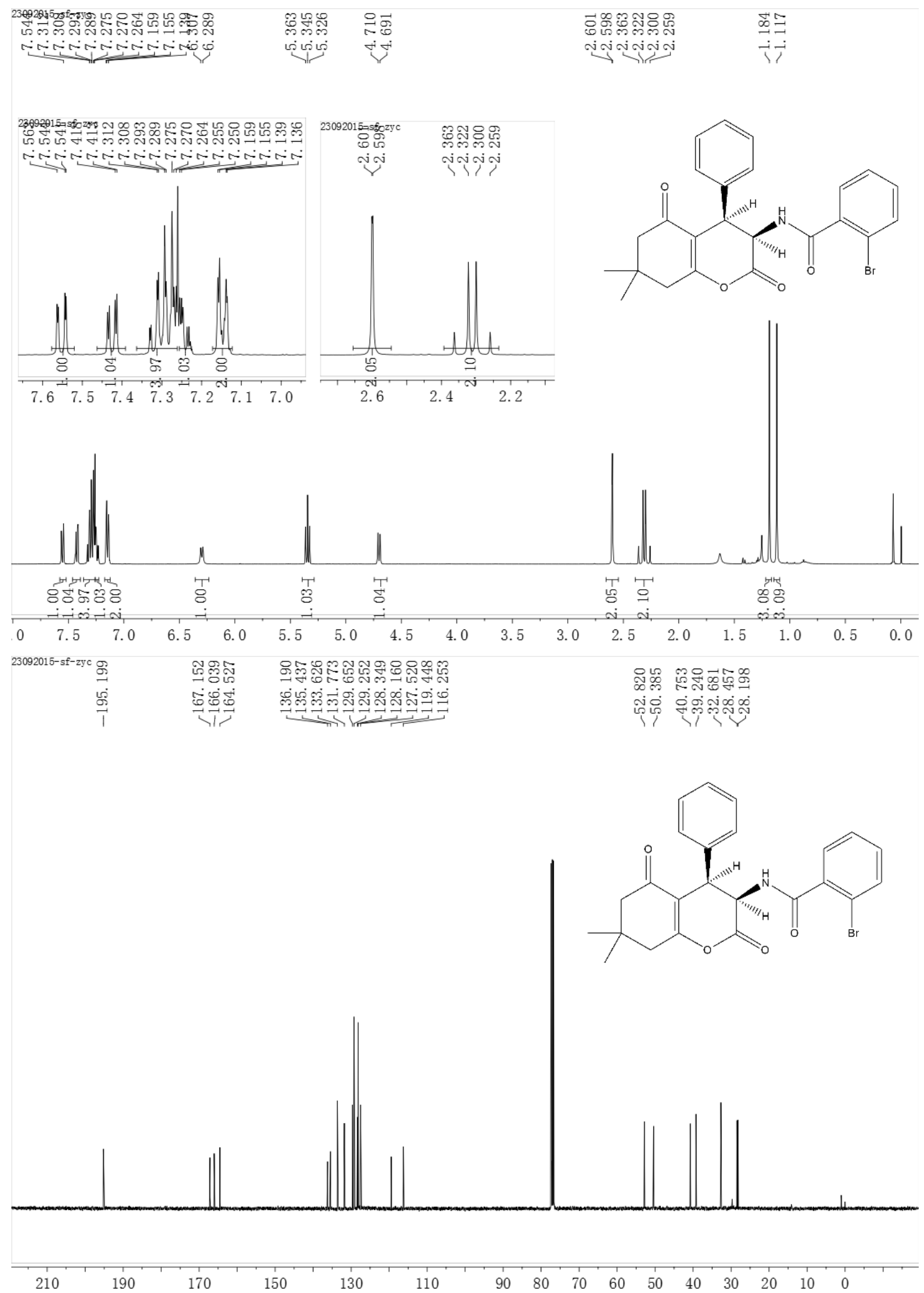
3da

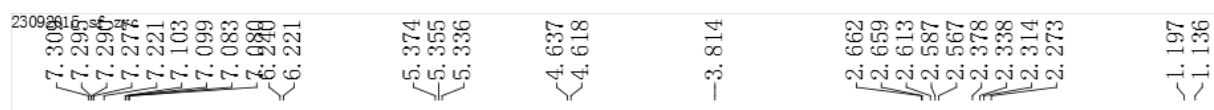

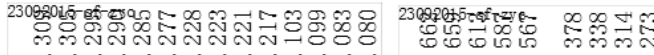

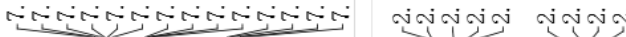
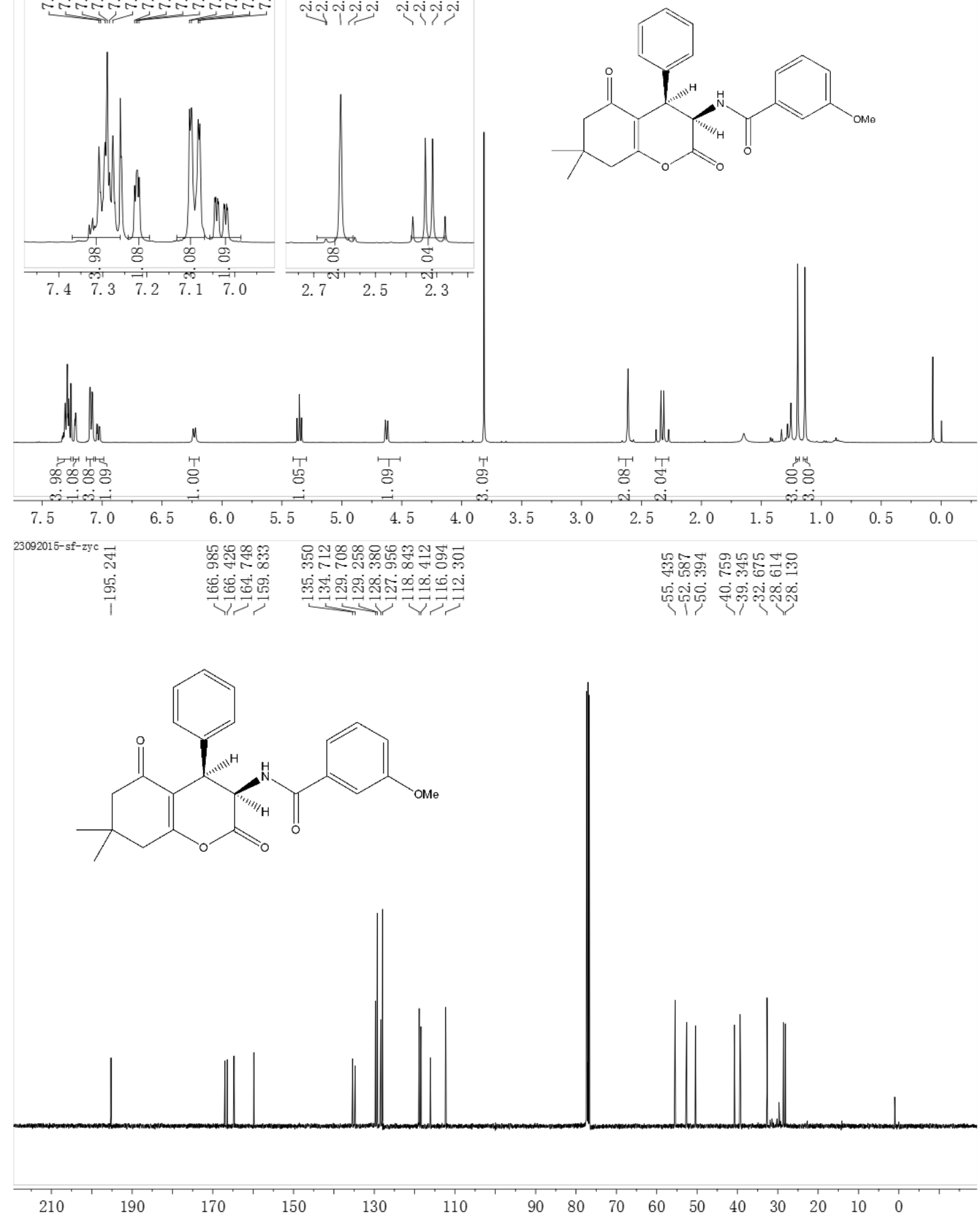


\section{3ea}
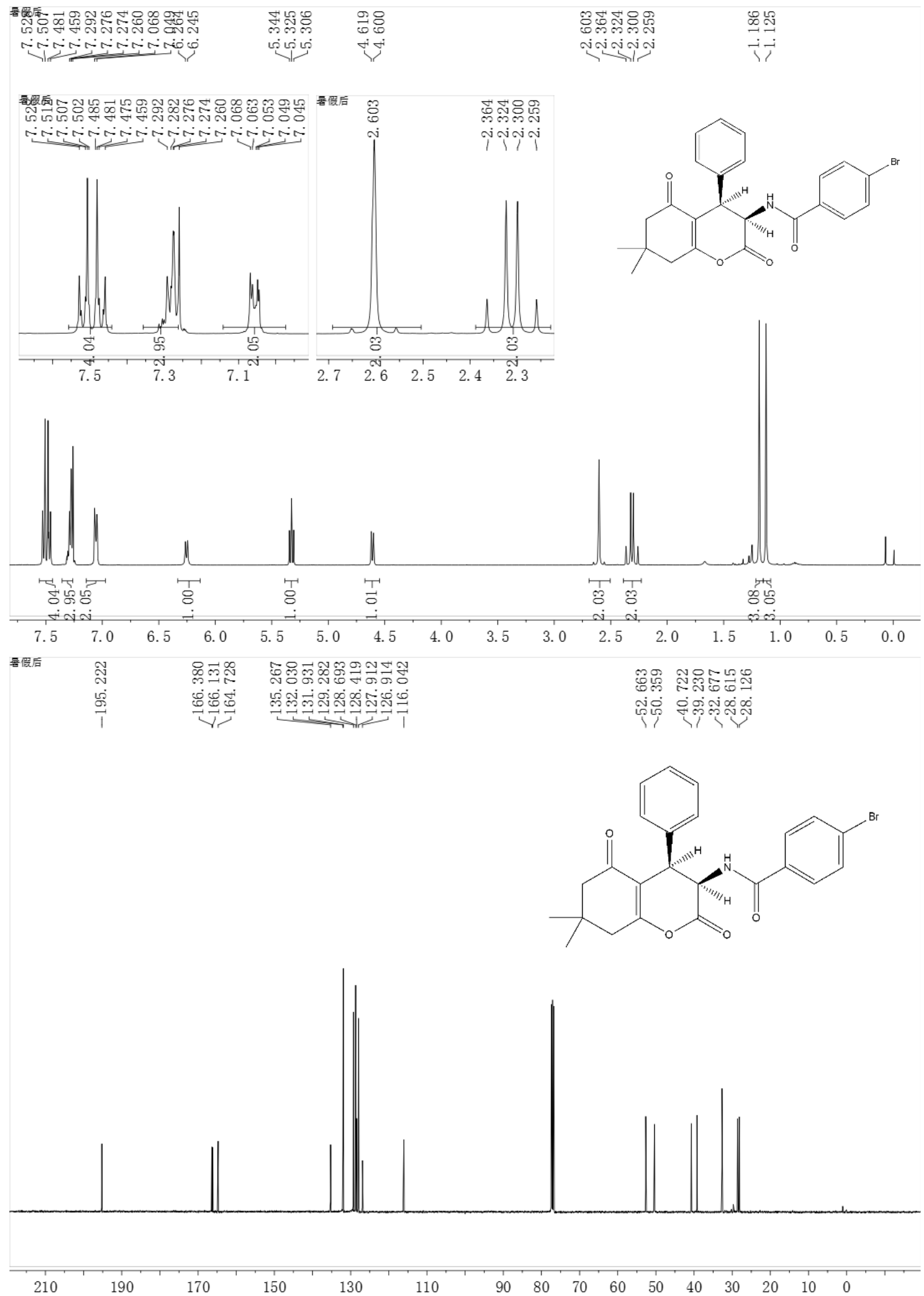


\section{3fa}
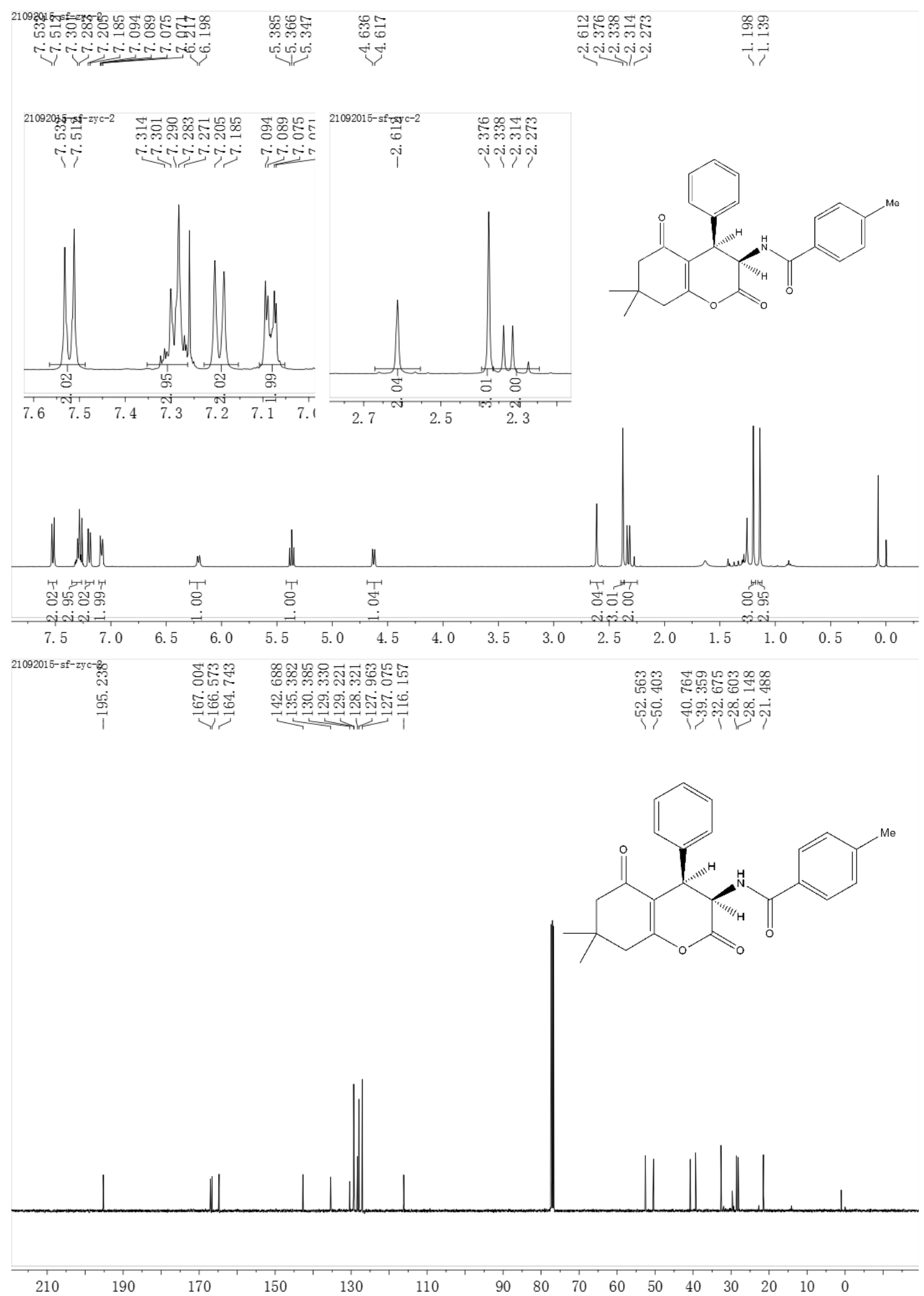


\section{3 ga}
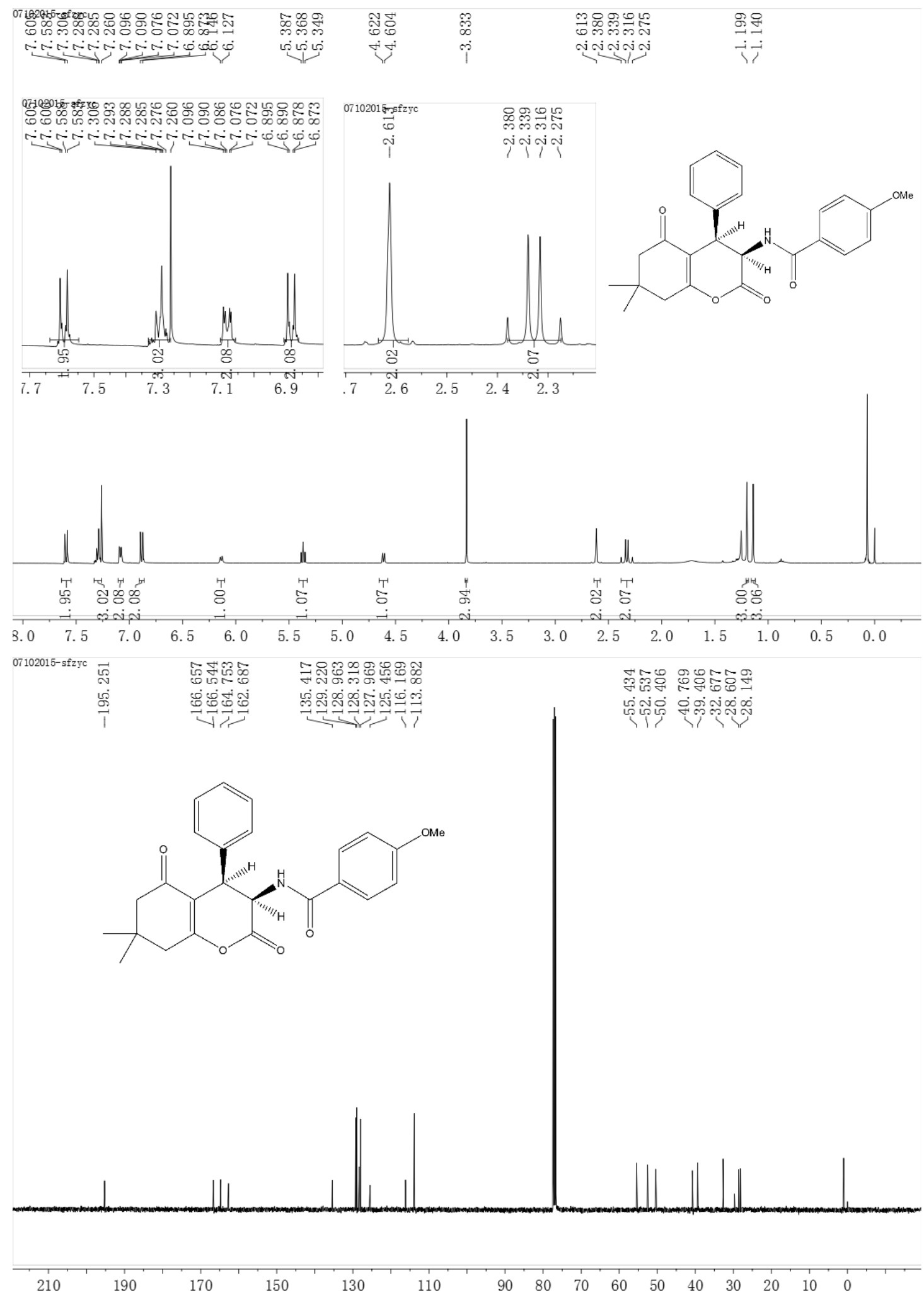


\section{3ha}

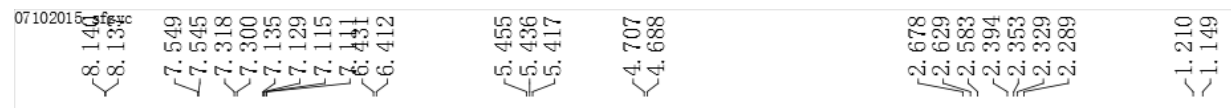
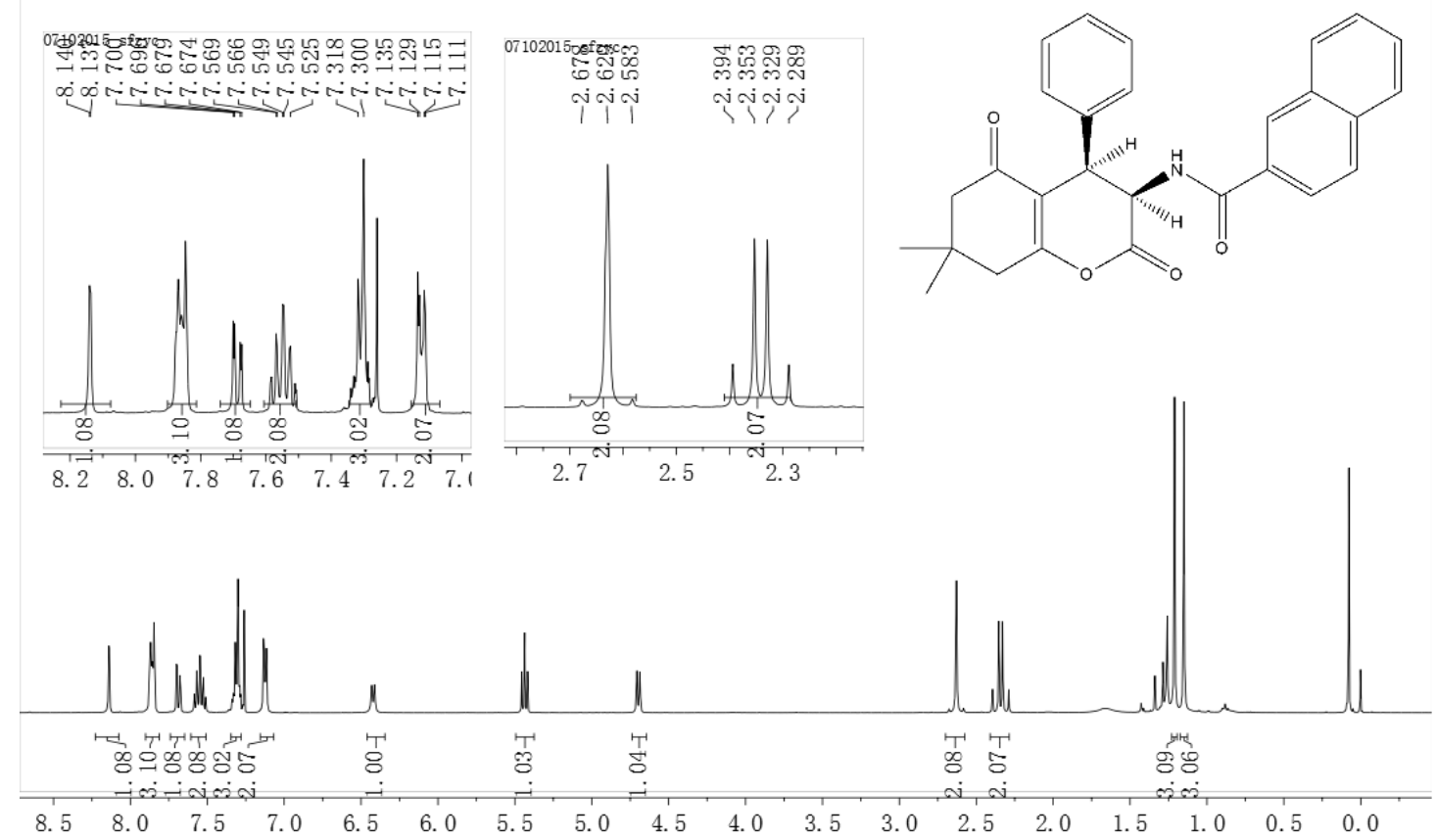

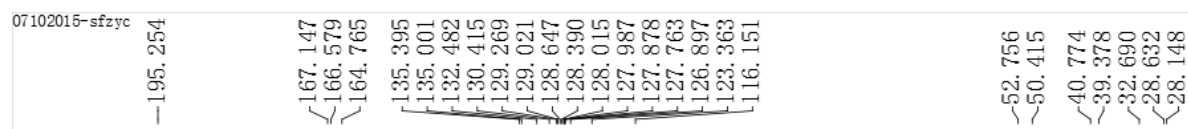<smiles>CC1(C)CC(=O)C2=C(C1)OC(=O)[C@](C)(NC(=O)c1ccc3ccccc3c1)[C@H]2c1ccccc1</smiles>

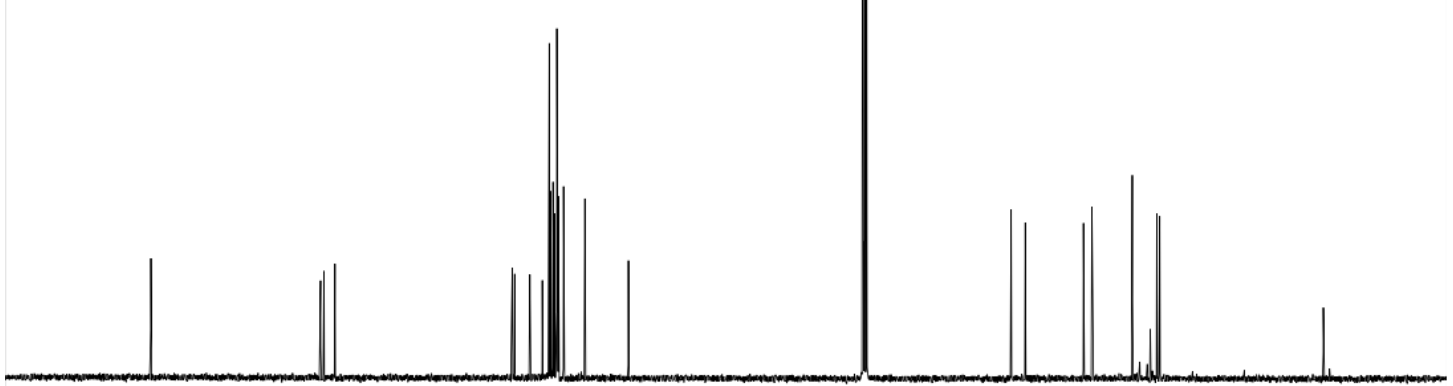

$\begin{array}{lllllllllllllllll}1 & 1 & 190 & 170 & 150 & 130 & 110 & 90 & 80 & 70 & 60 & 50 & 40 & 30 & 20 & 10 & 0\end{array}$ 
3ia

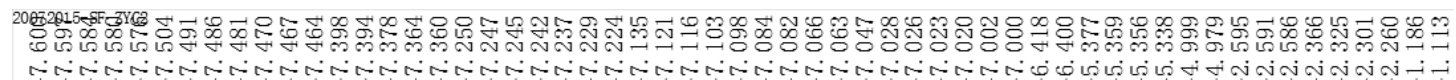

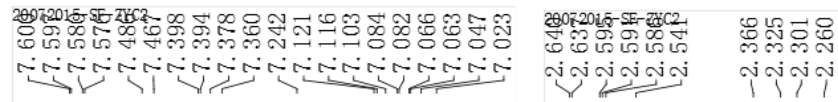
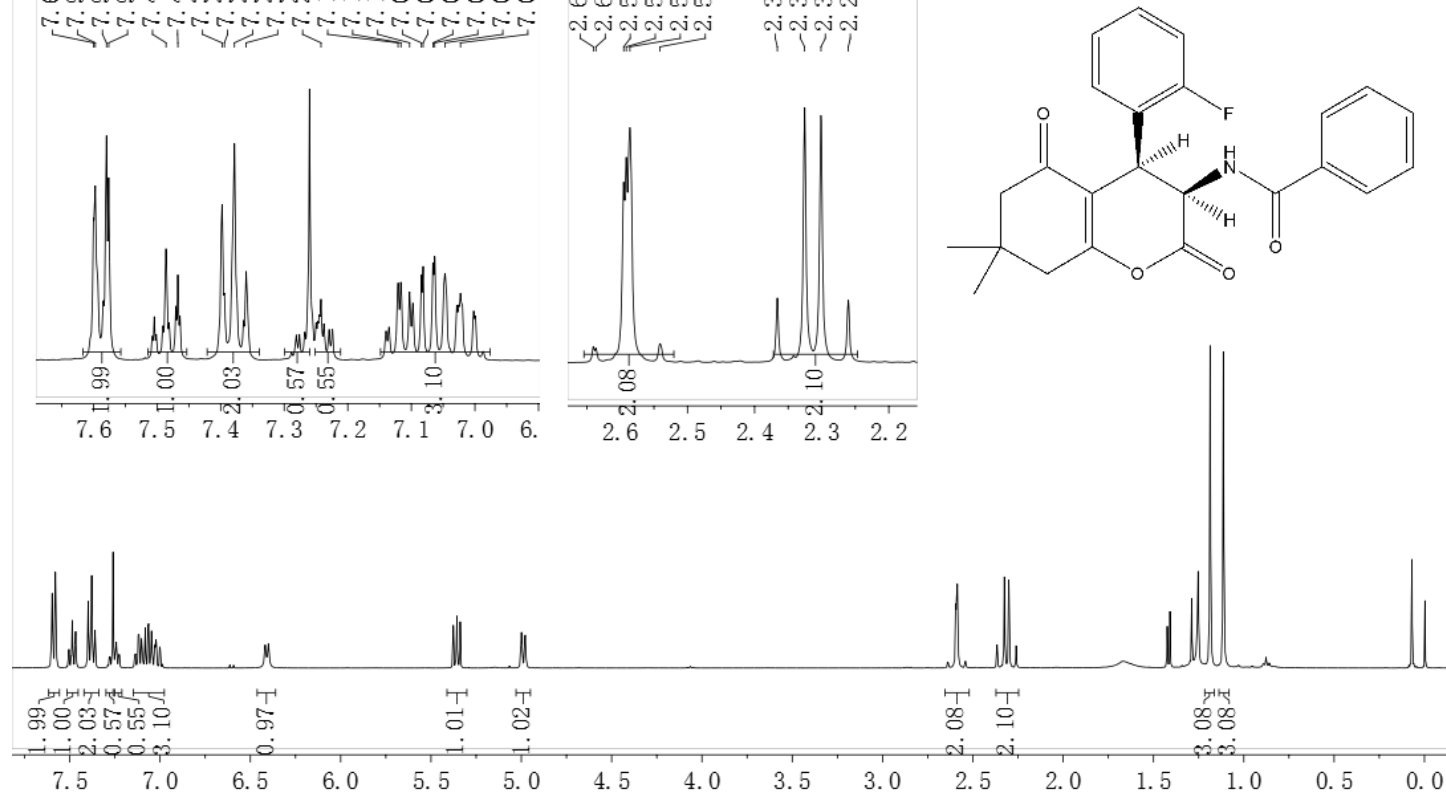

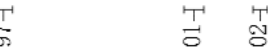

宛军

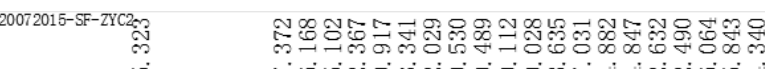

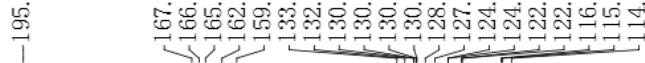<smiles>CC1(C)CC(=O)C2=C(C1)OC(=O)[C@](C)(NC(=O)c1ccccc1)[C@]2(F)c1ccccc1F</smiles>

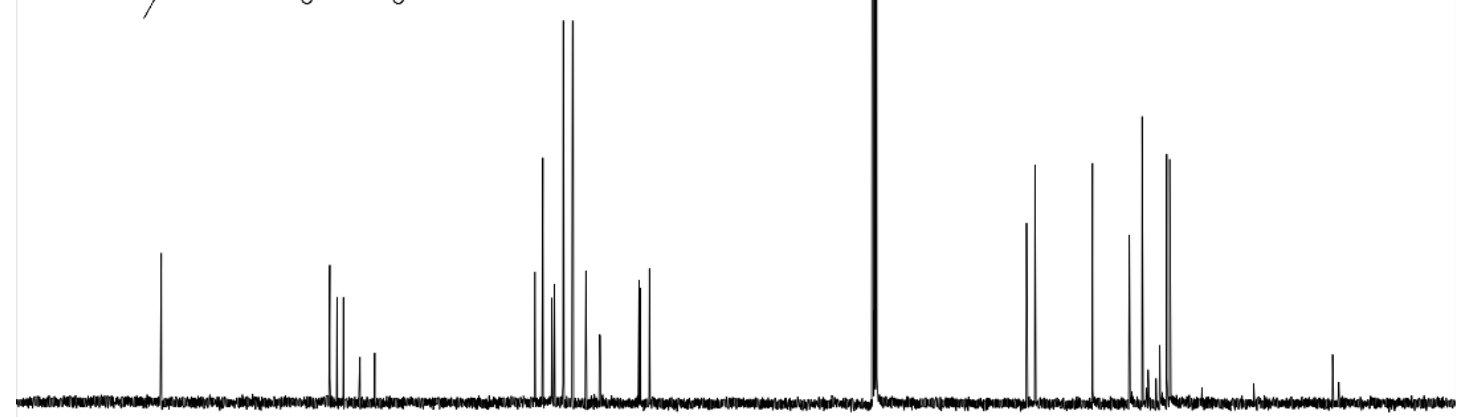

210

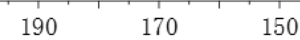

130

110

$90 \quad 80$

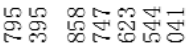

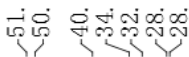

trty

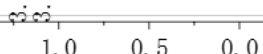




\section{3ja}

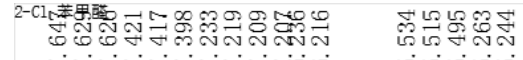

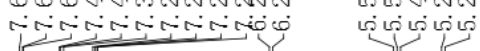

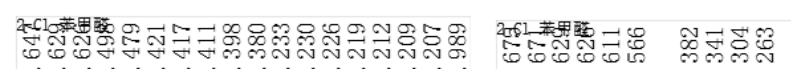

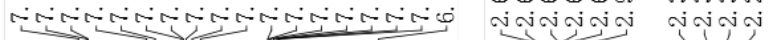
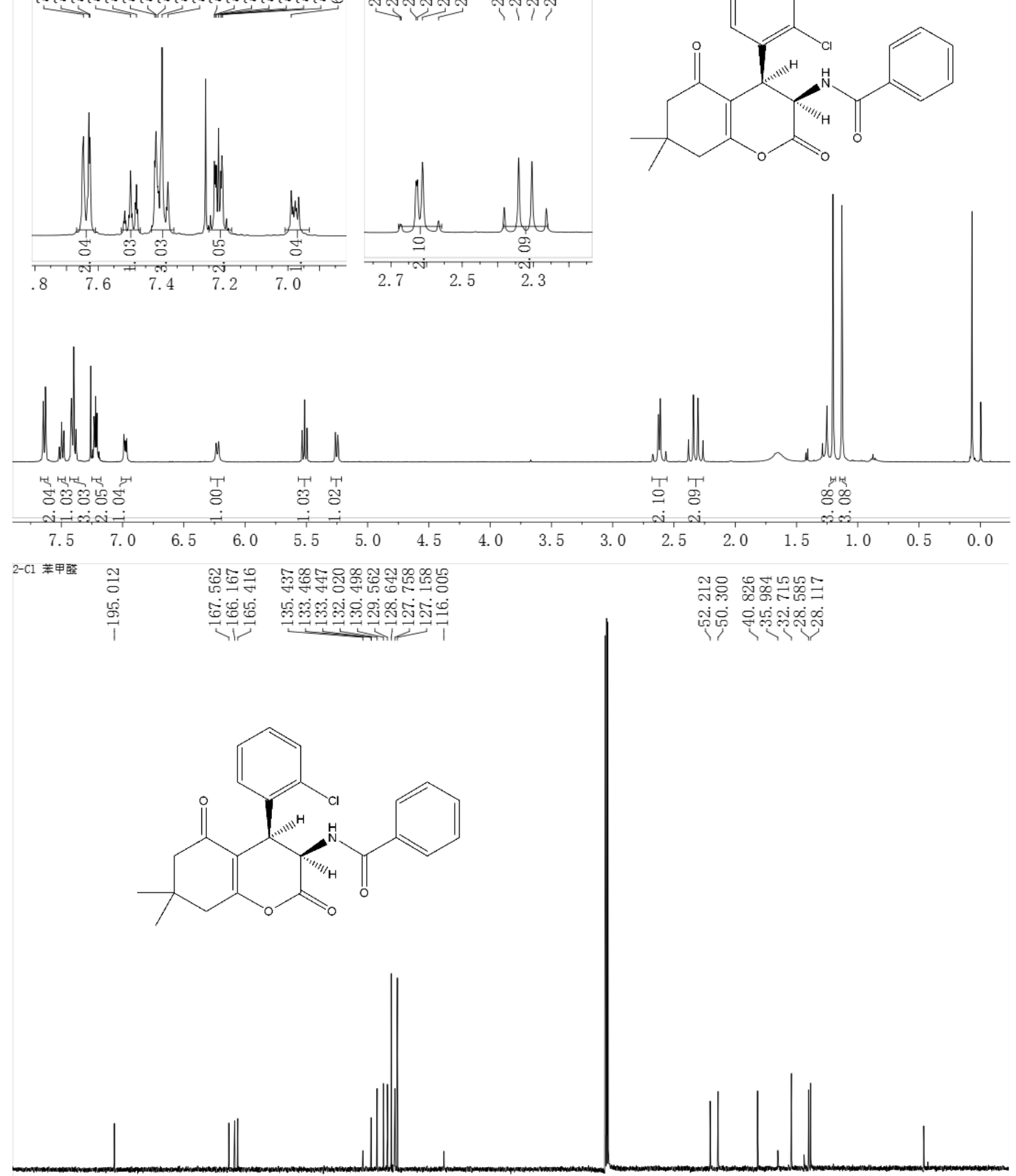

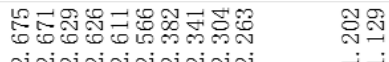

ค่งiniaiaia

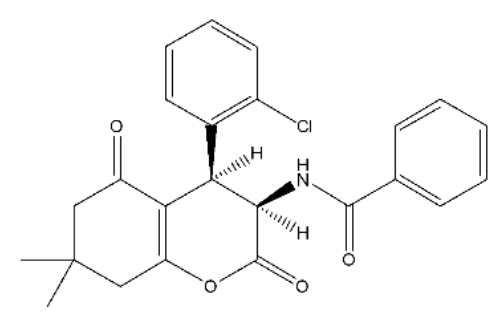

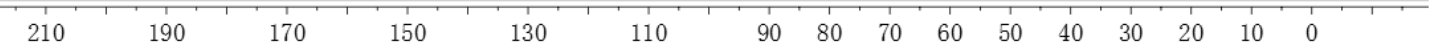


3ka

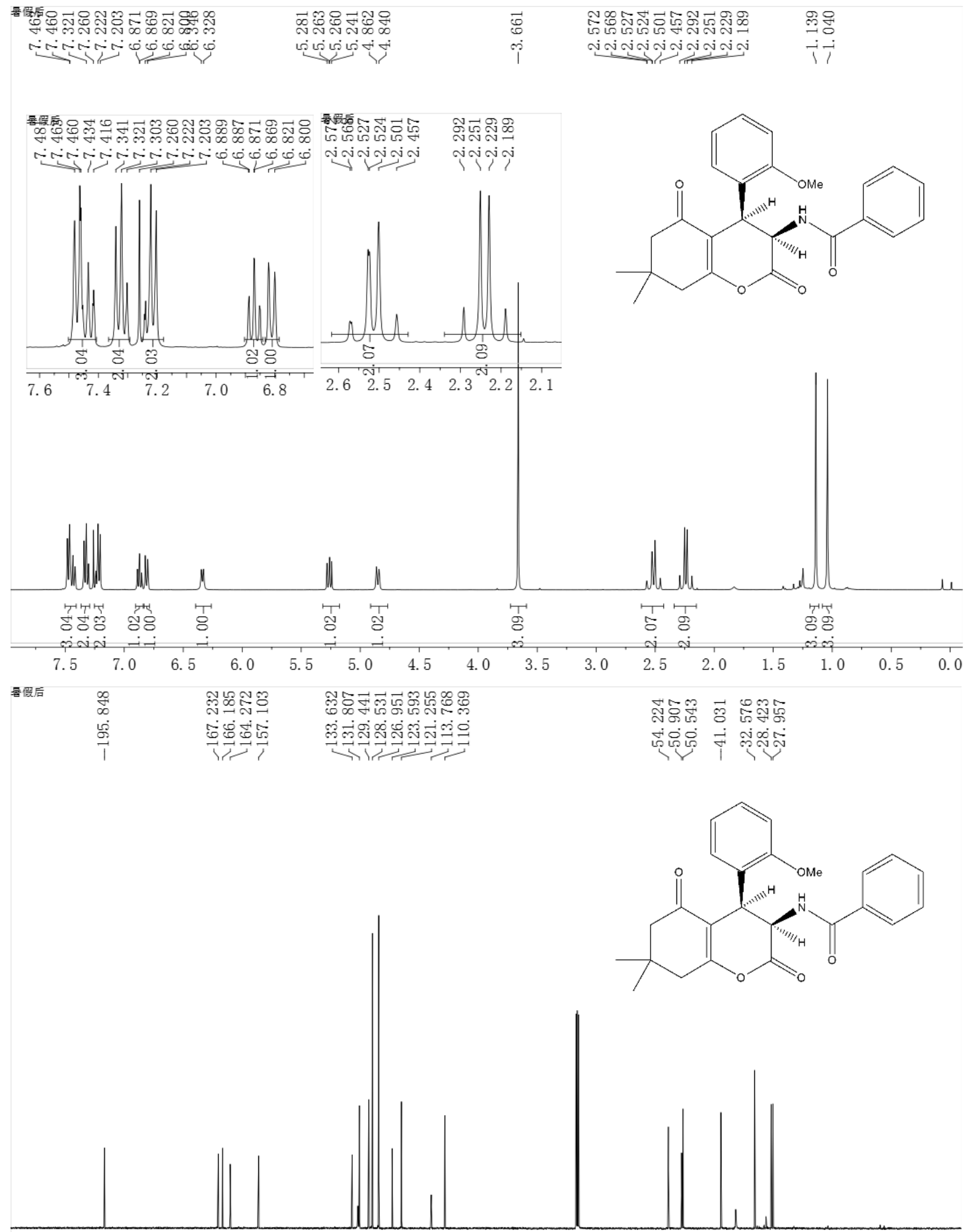

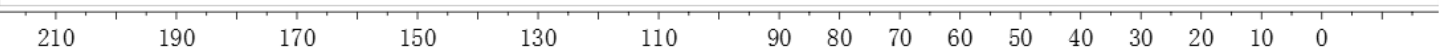


3la

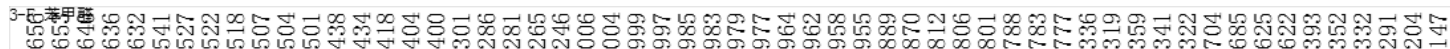

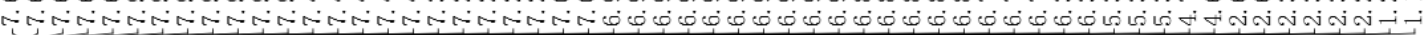
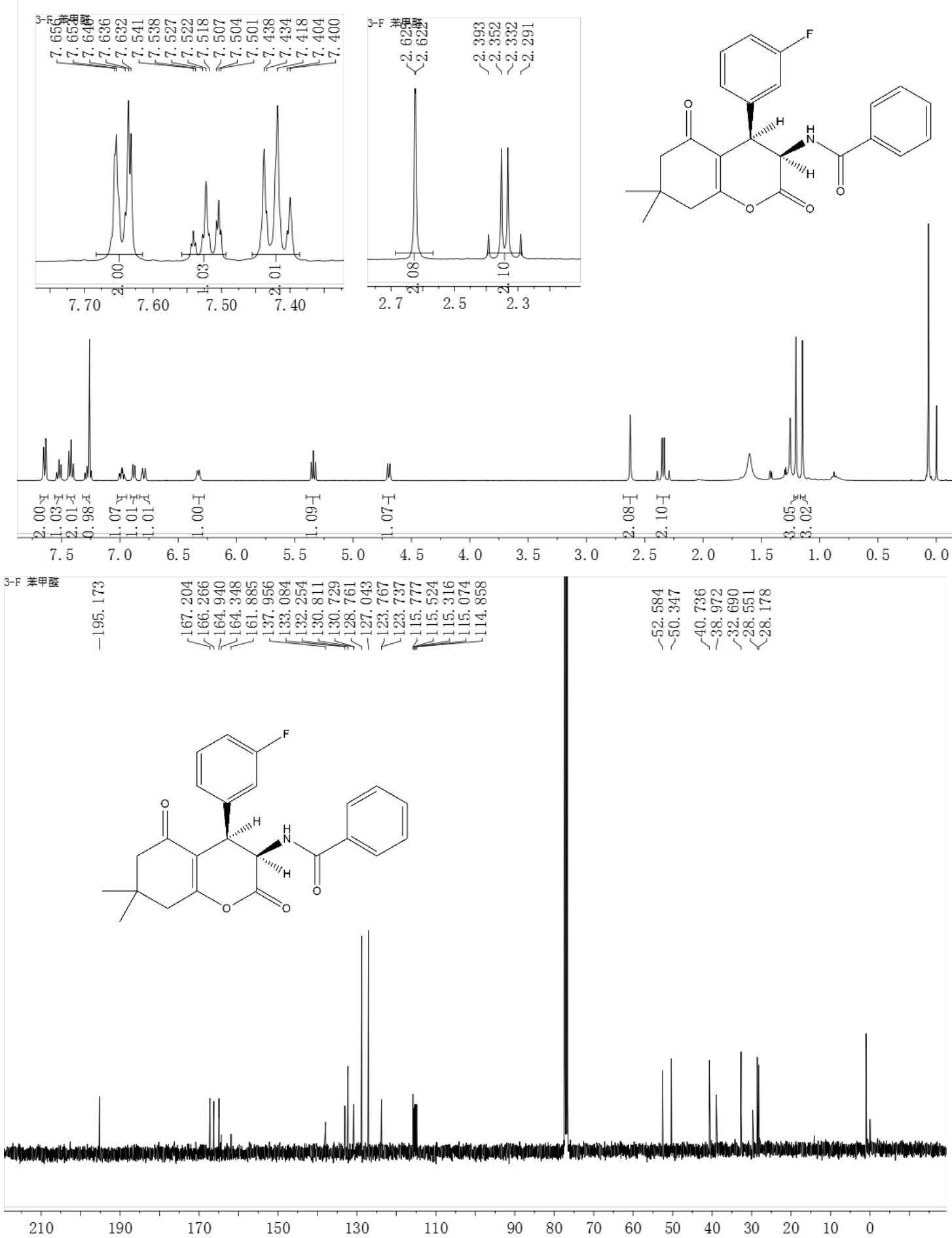

$10+190$

150

130

9080

닝

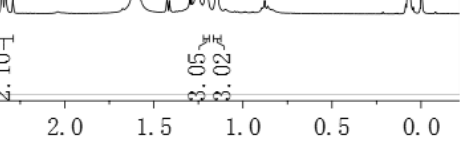

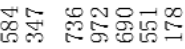

งิ 


\section{3ma}

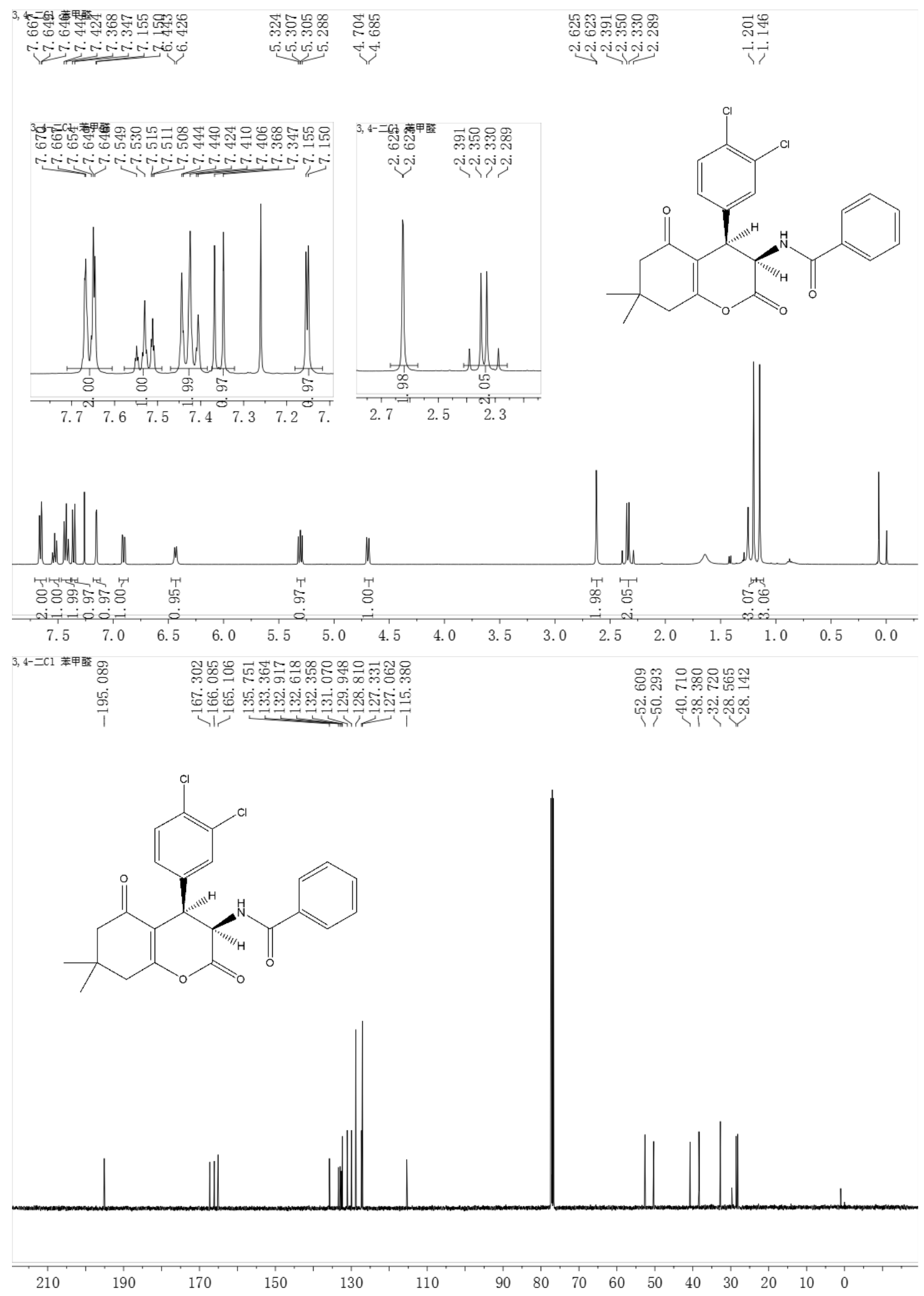




\section{3na}

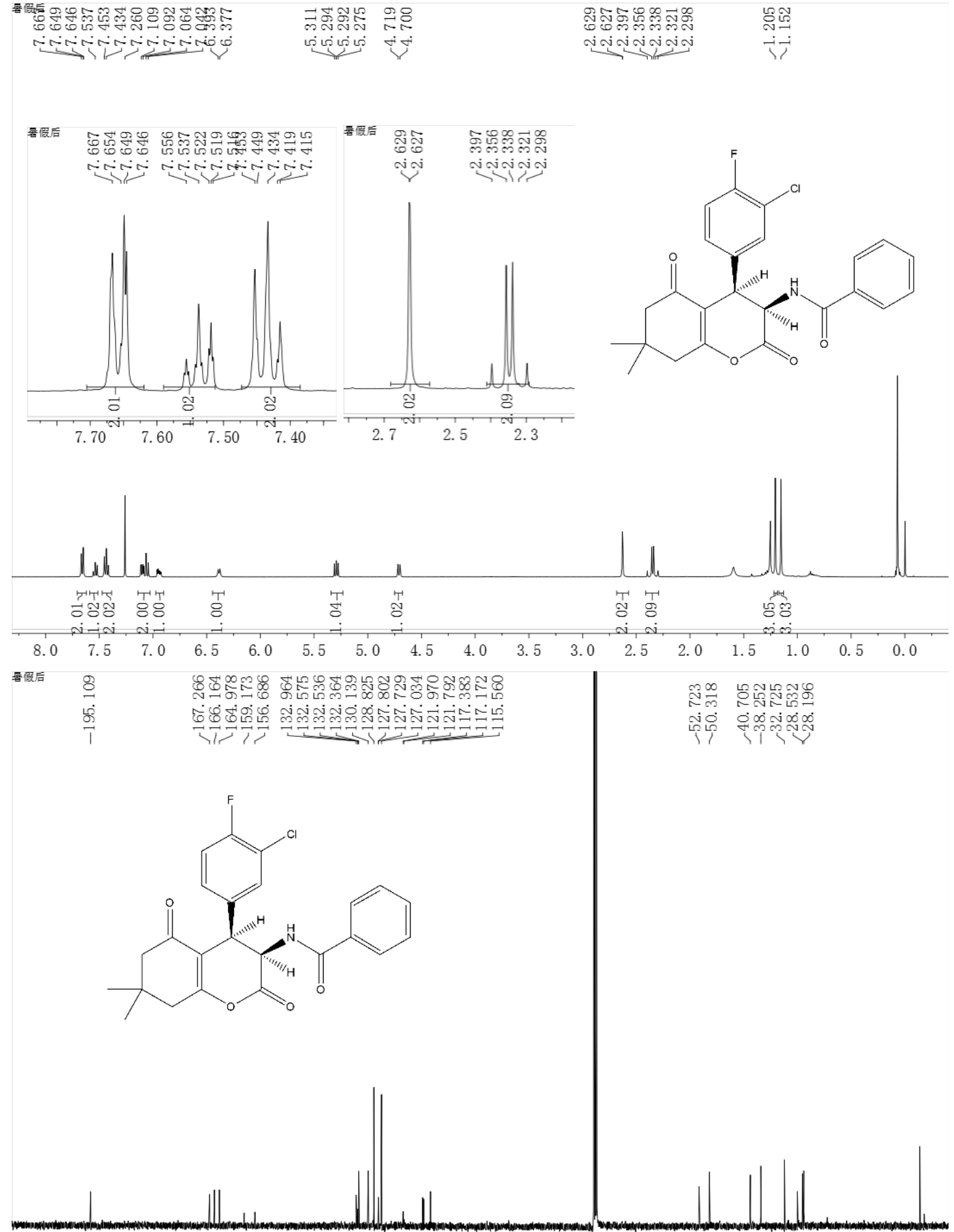

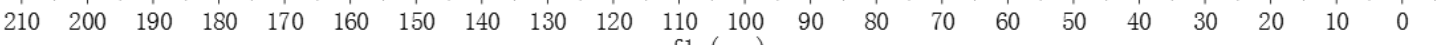




\section{3oa}

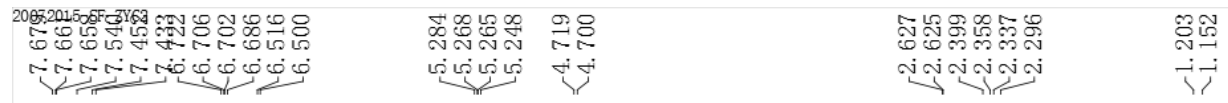
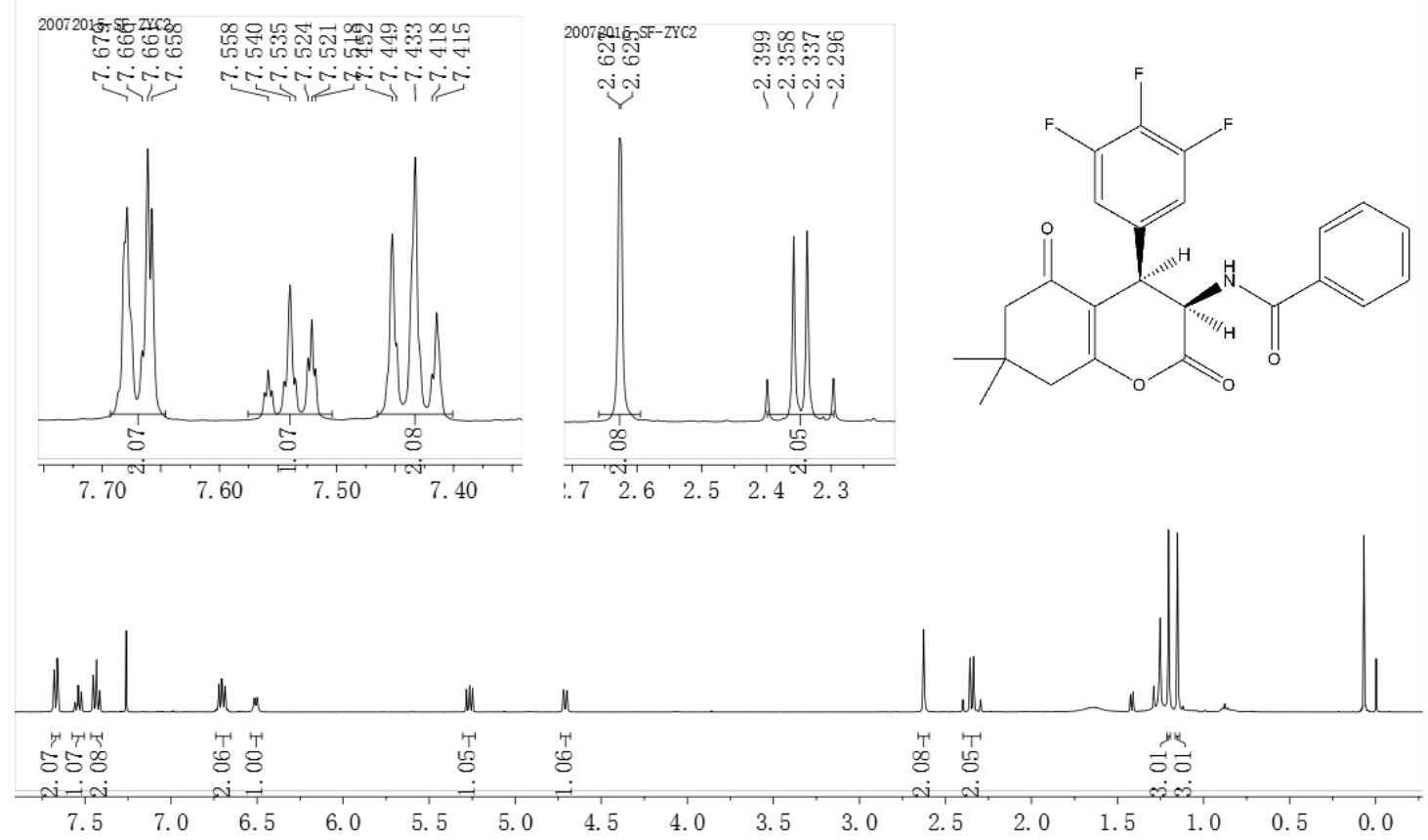

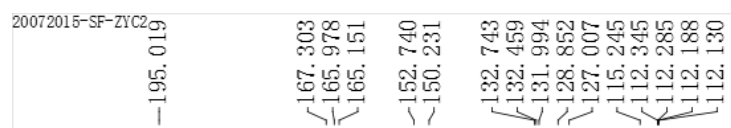<smiles>Cc1cc([C@]2(NC(=O)c3ccccc3)C(=O)OC3=C(C(=O)CC(C)(C)C3)C2(C)C)cc(F)c1F</smiles>

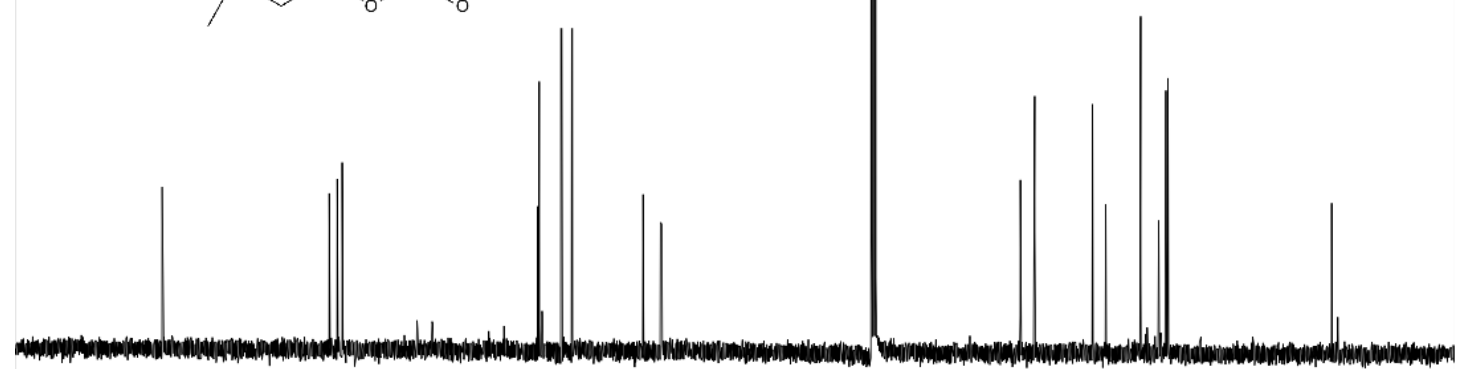

$\begin{array}{llllllllllllllll}210 & 190 & 170 & 150 & 130 & 110 & 90 & 80 & 70 & 60 & 50 & 40 & 30 & 20 & 10 & 0\end{array}$ 


\section{Зра}

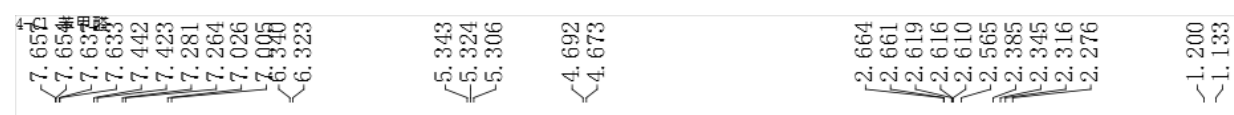

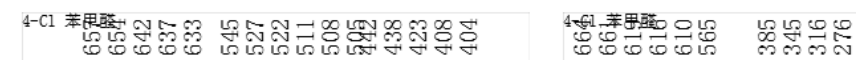

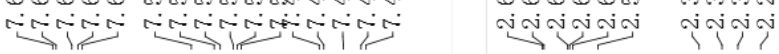
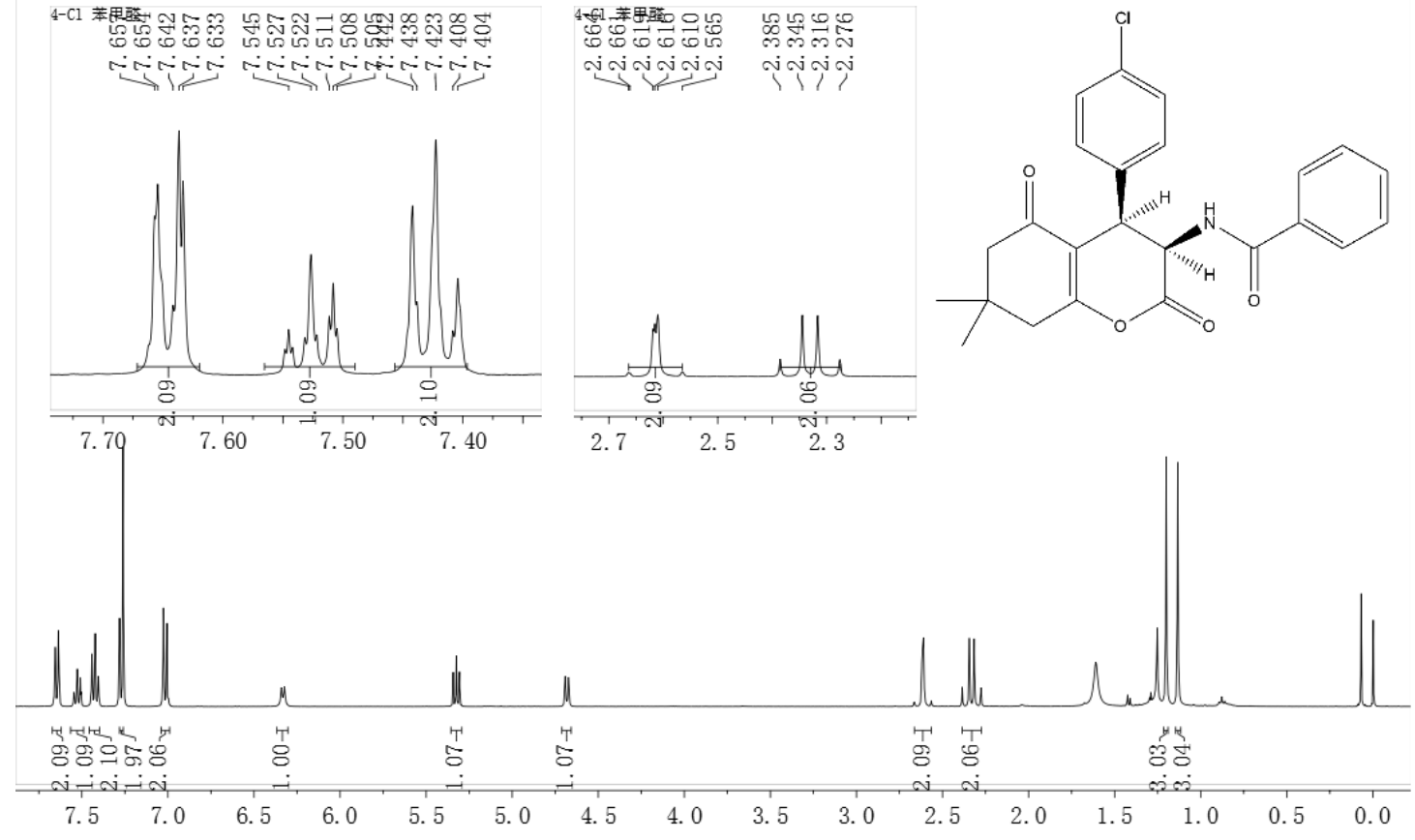

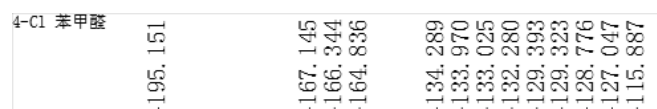

।<smiles>CC1(C)CC(=O)C2=C(C1)OC(=O)[C@H](NC(=O)c1ccccc1)[C@H]2c1ccc(Cl)cc1</smiles>
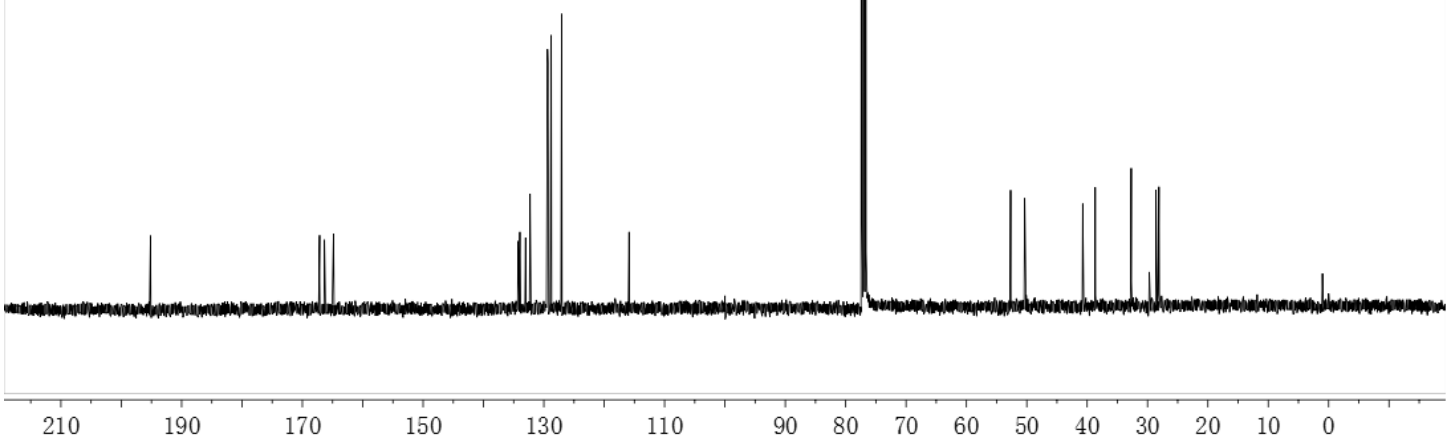


\section{3qa}
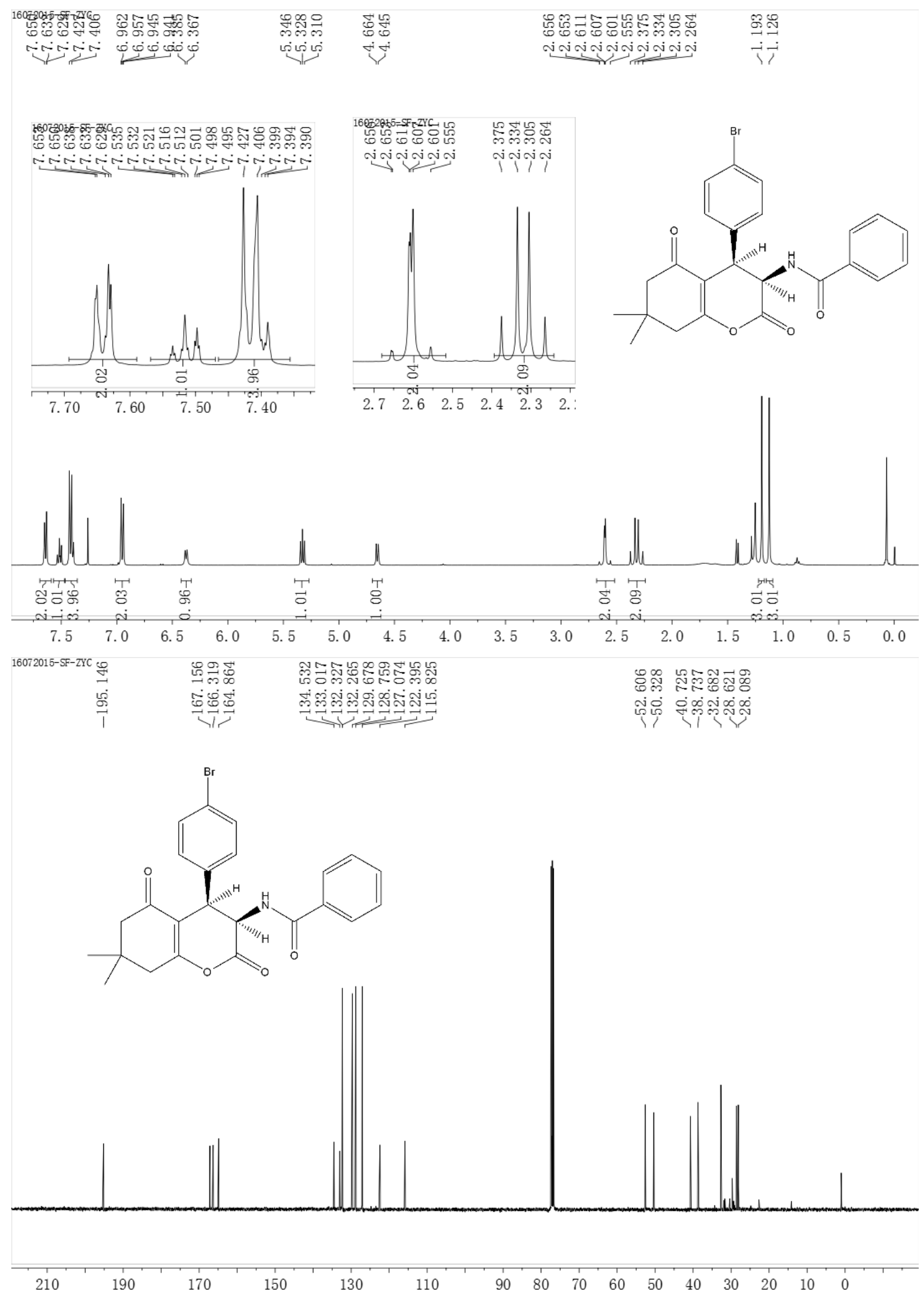


\section{3ra}
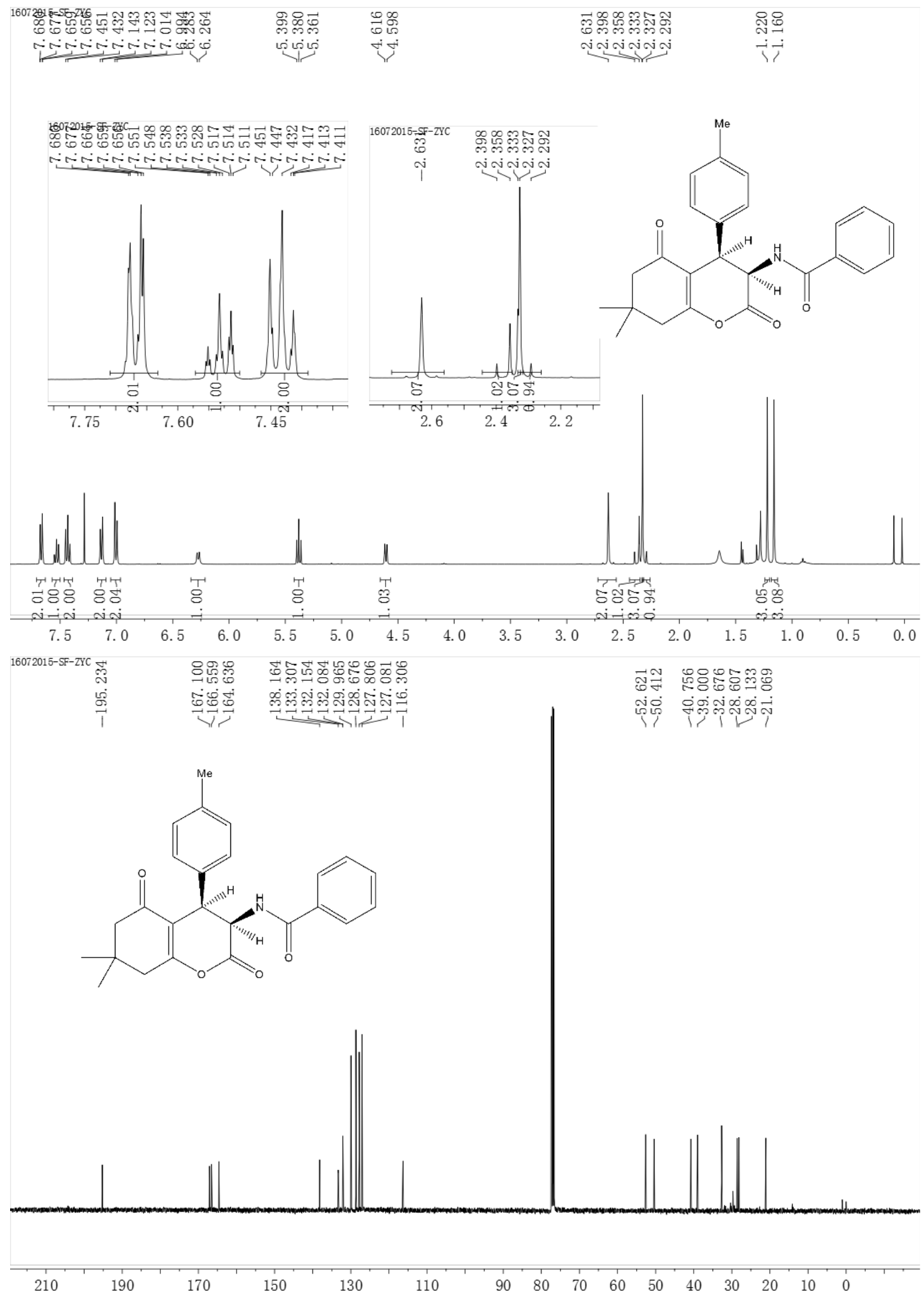


\section{3sa}

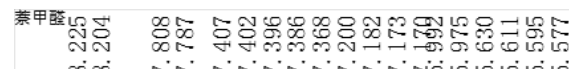

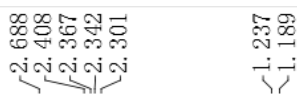

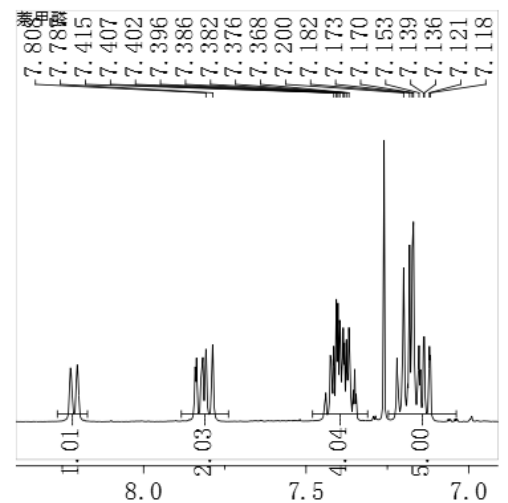

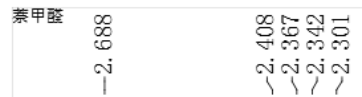
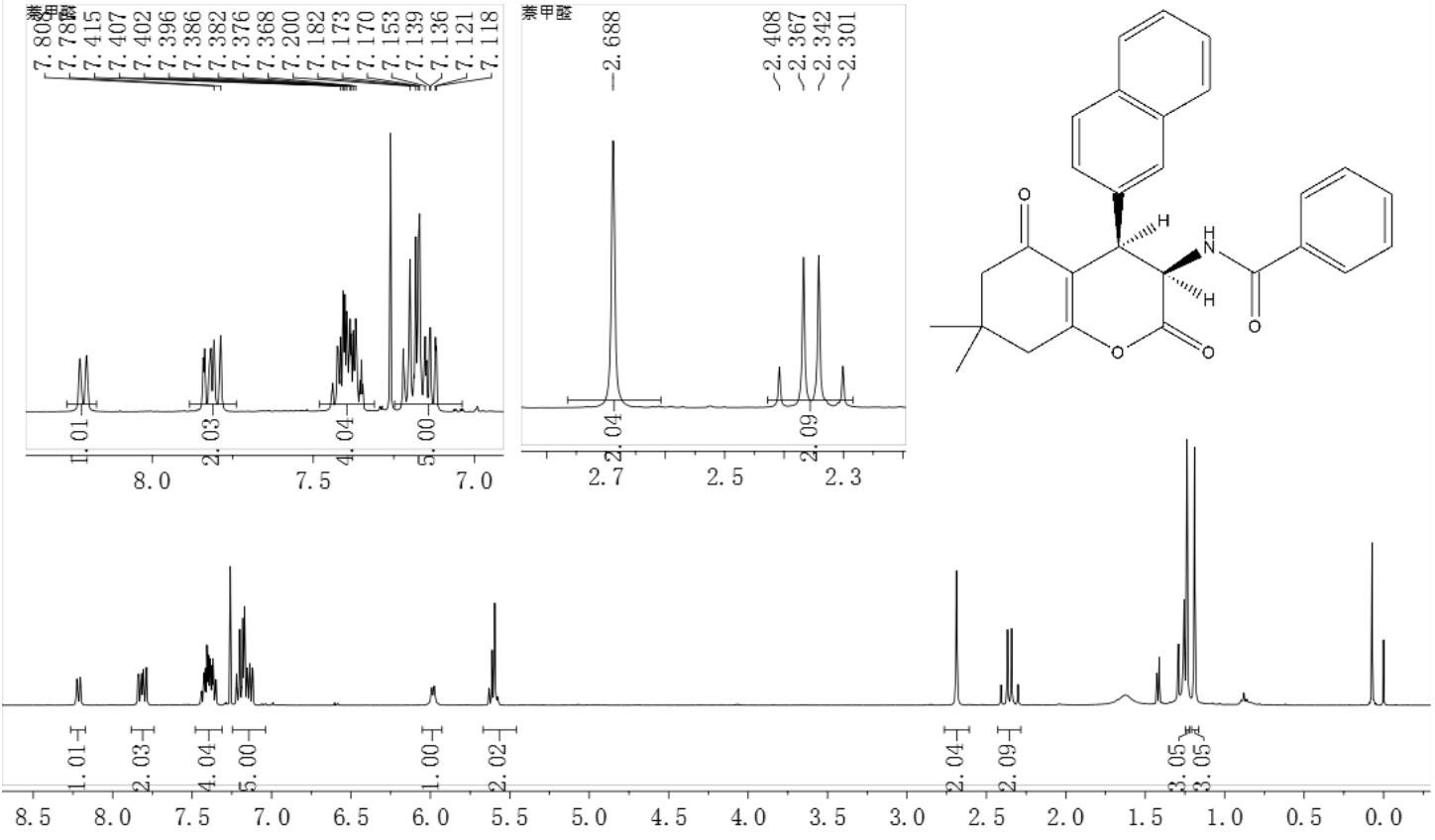

暮甲酸

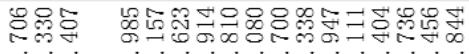

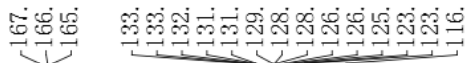

구유

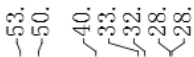<smiles>[CH][C@@]1(c2ccc3ccccc3c2)C2=C(CC(C)(C)CC2=O)OC(=O)[C@@H]1NC(=O)c1ccccc1</smiles>

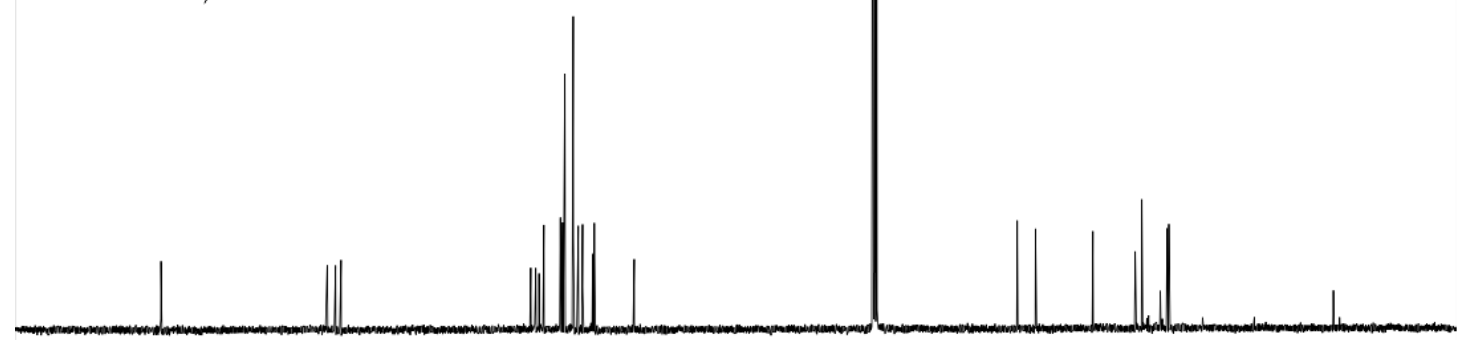

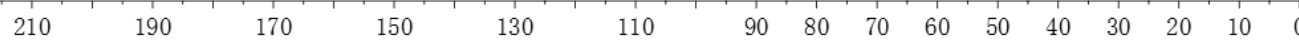


3ta

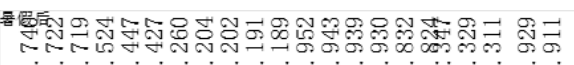

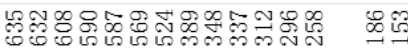

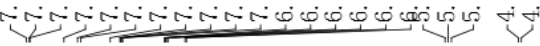

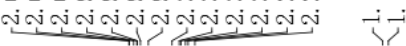

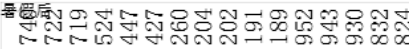

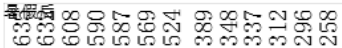

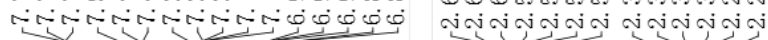
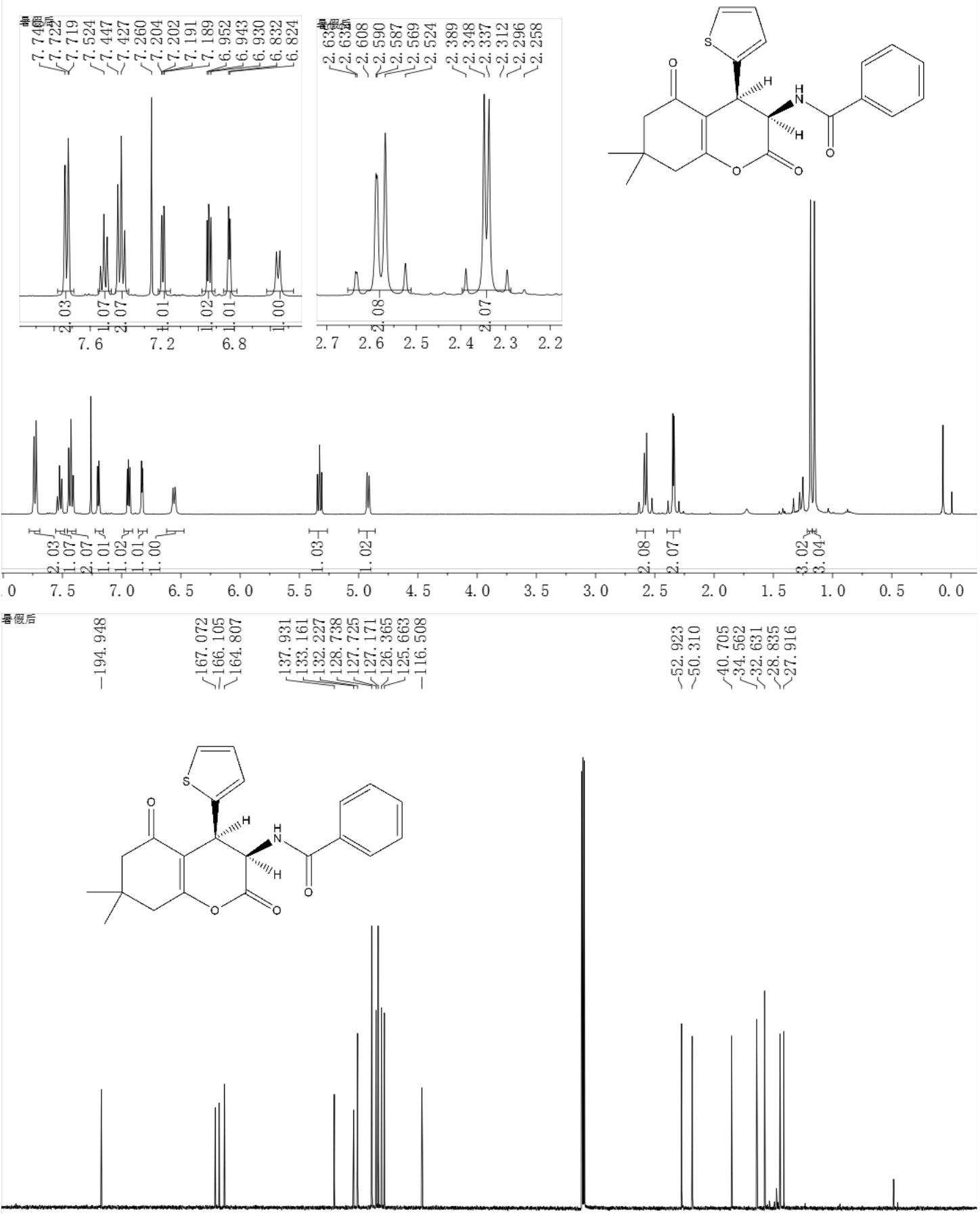

210

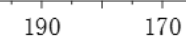

150

130

9080

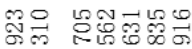

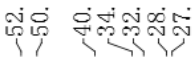

装若

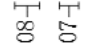

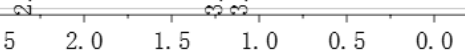


3ab

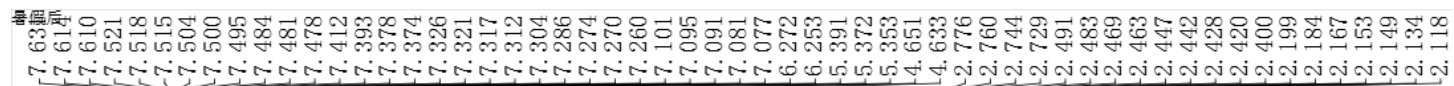

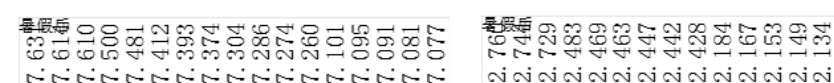

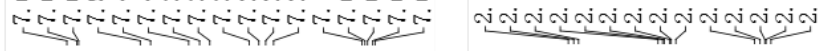
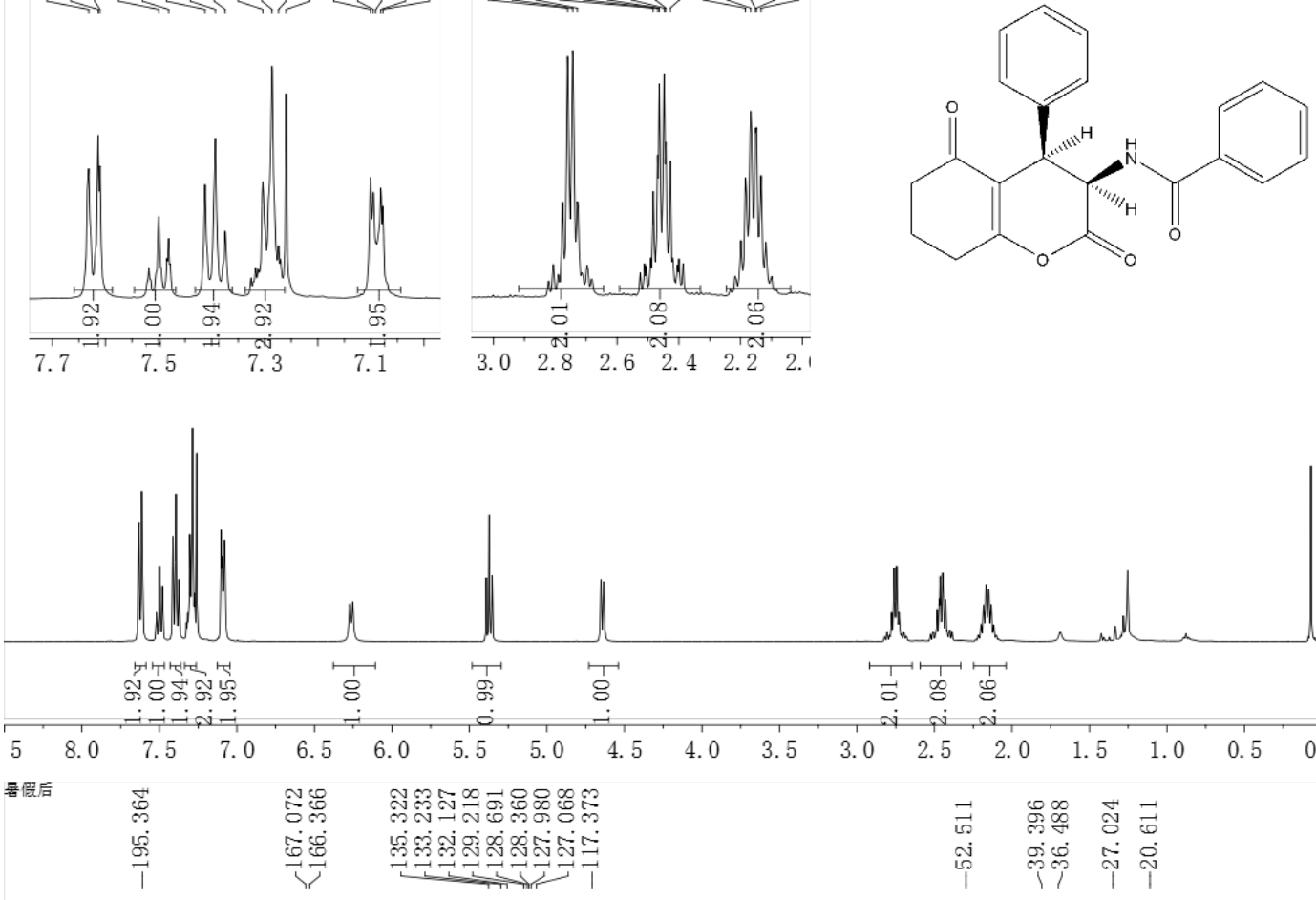<smiles>O=C1CCCC2=C1[C@H](c1ccccc1)[C@H](NC(=O)c1ccccc1)C(=O)O2</smiles>
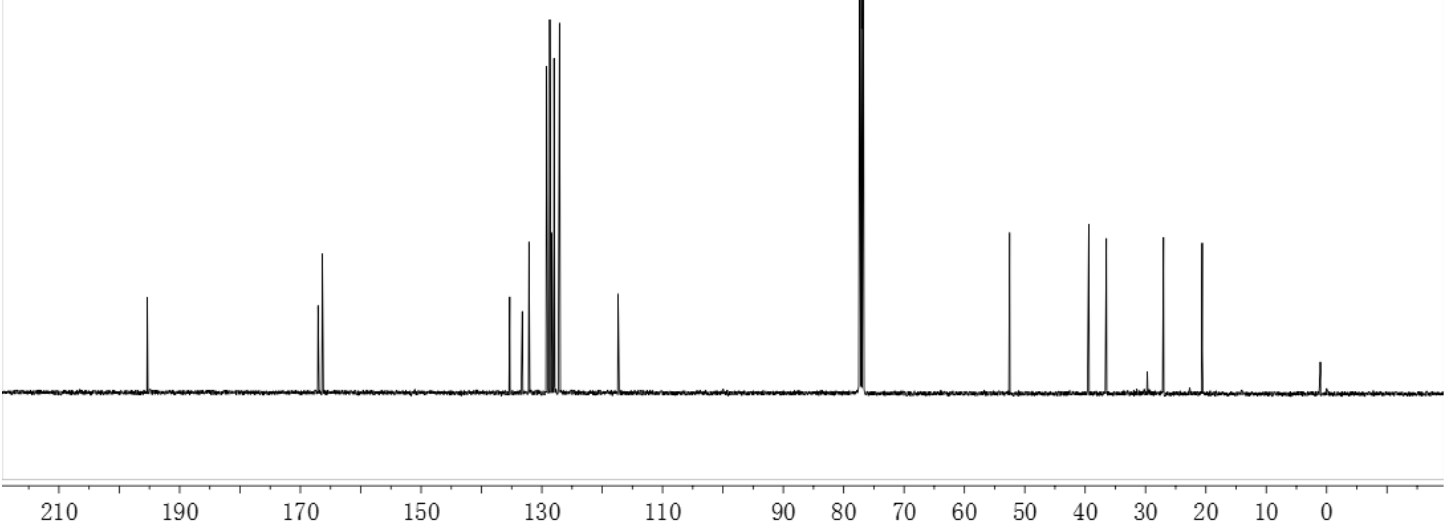


\section{3ad}

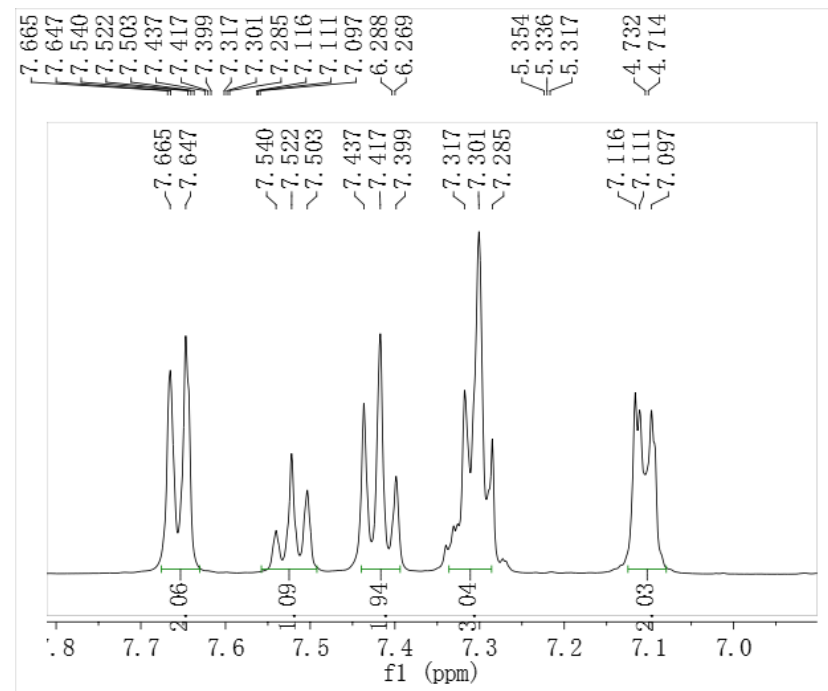

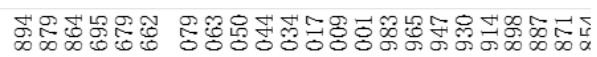

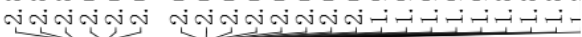
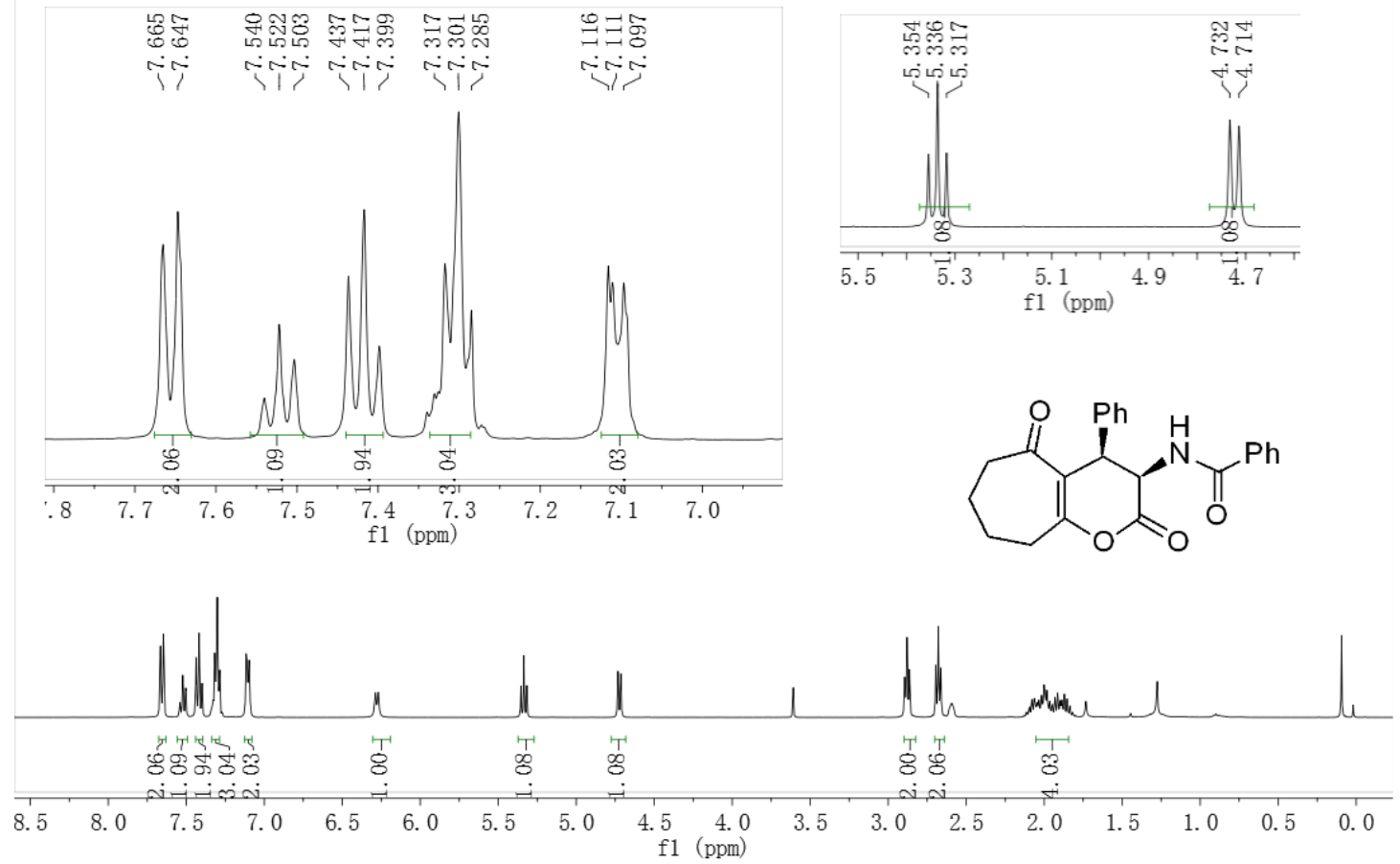

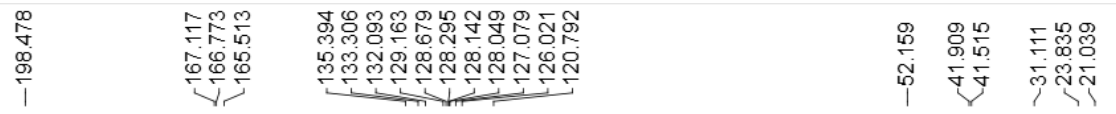<smiles>O=C1CCCCC2=C1[C@](Cc1ccccc1)(NC(=O)c1ccccc1)C(=O)O2</smiles>

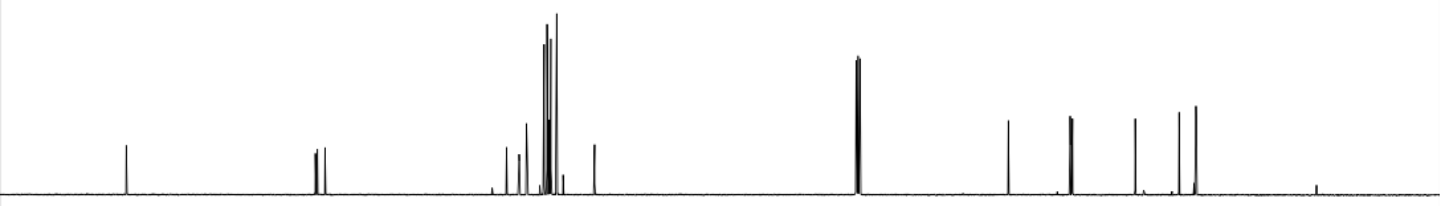

$\begin{array}{lllllllllllllllllllllll}210 & 200 & 190 & 180 & 170 & 160 & 150 & 140 & 130 & 120 & 110 & 100 & 90 & 80 & 70 & 60 & 50 & 40 & 30 & 20 & 10 & 0 & -10\end{array}$ 


\section{3ae}

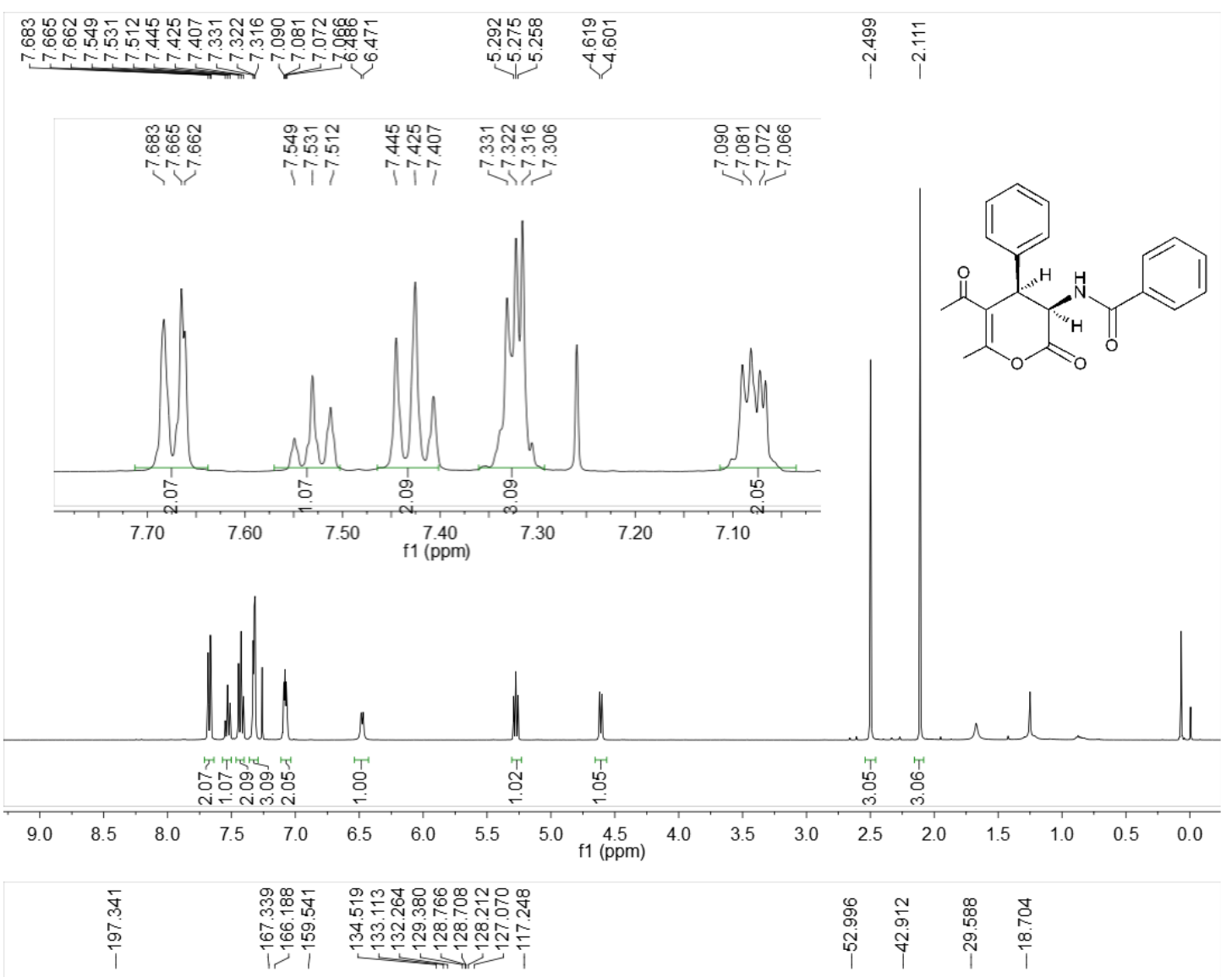<smiles>CC(=O)C1=C(C)OC(=O)[C@](NC(=O)c2ccccc2)(c2ccccc2)C1</smiles>

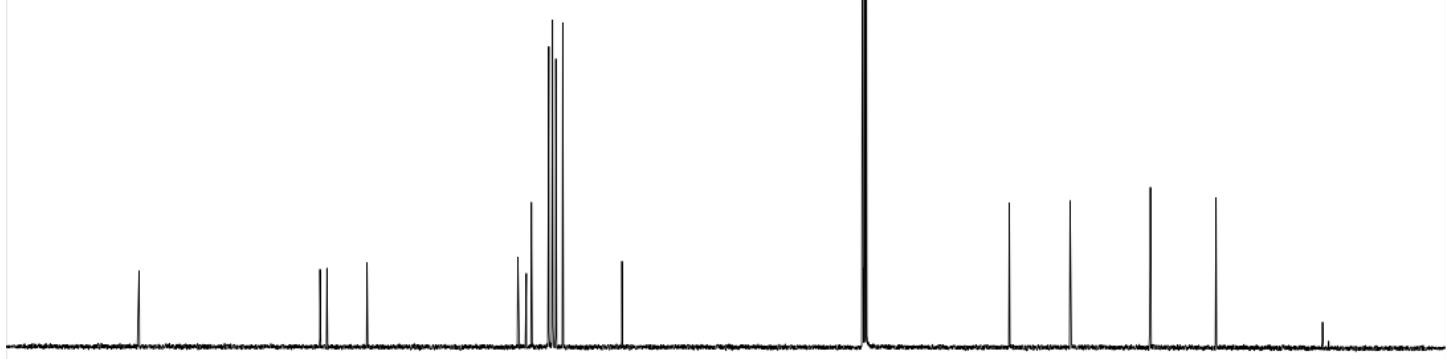

$\begin{array}{lllllllllllllllllllllll}210 & 200 & 190 & 180 & 170 & 160 & 150 & 140 & 130 & 120 & 110 & 100 & 90 & 80 & 70 & 60 & 50 & 40 & 30 & 20 & 10 & 0 & -10\end{array}$ 


\section{Compound 5}

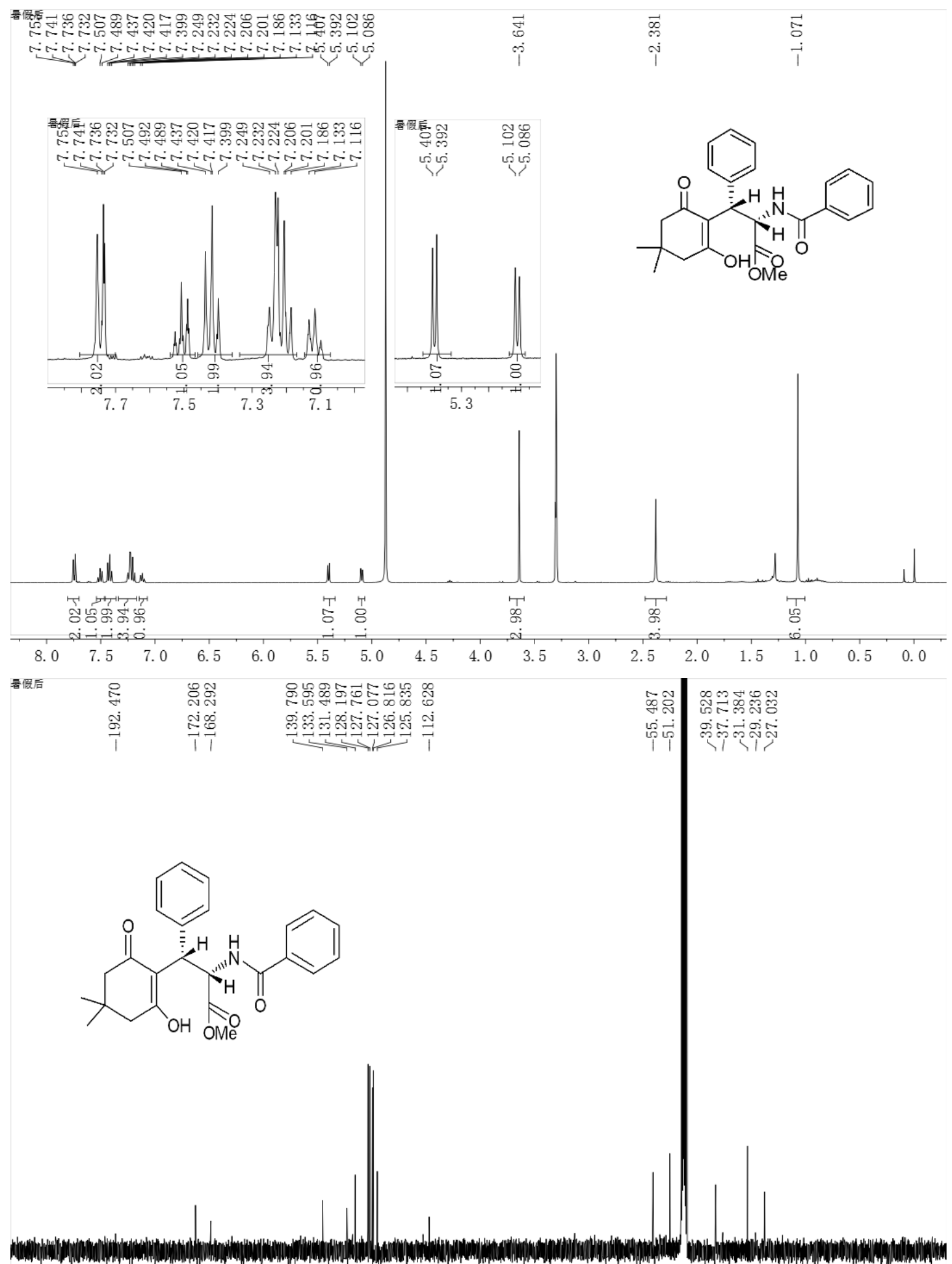

130

110

$\begin{array}{lllll}90 & 80 & 70 & 60 & 50\end{array}$

$\begin{array}{llll}30 & 20 & 10 & 0\end{array}$ 


\section{HPLC Traces of products 3 and 5}

\section{Заa}

Racemic product:

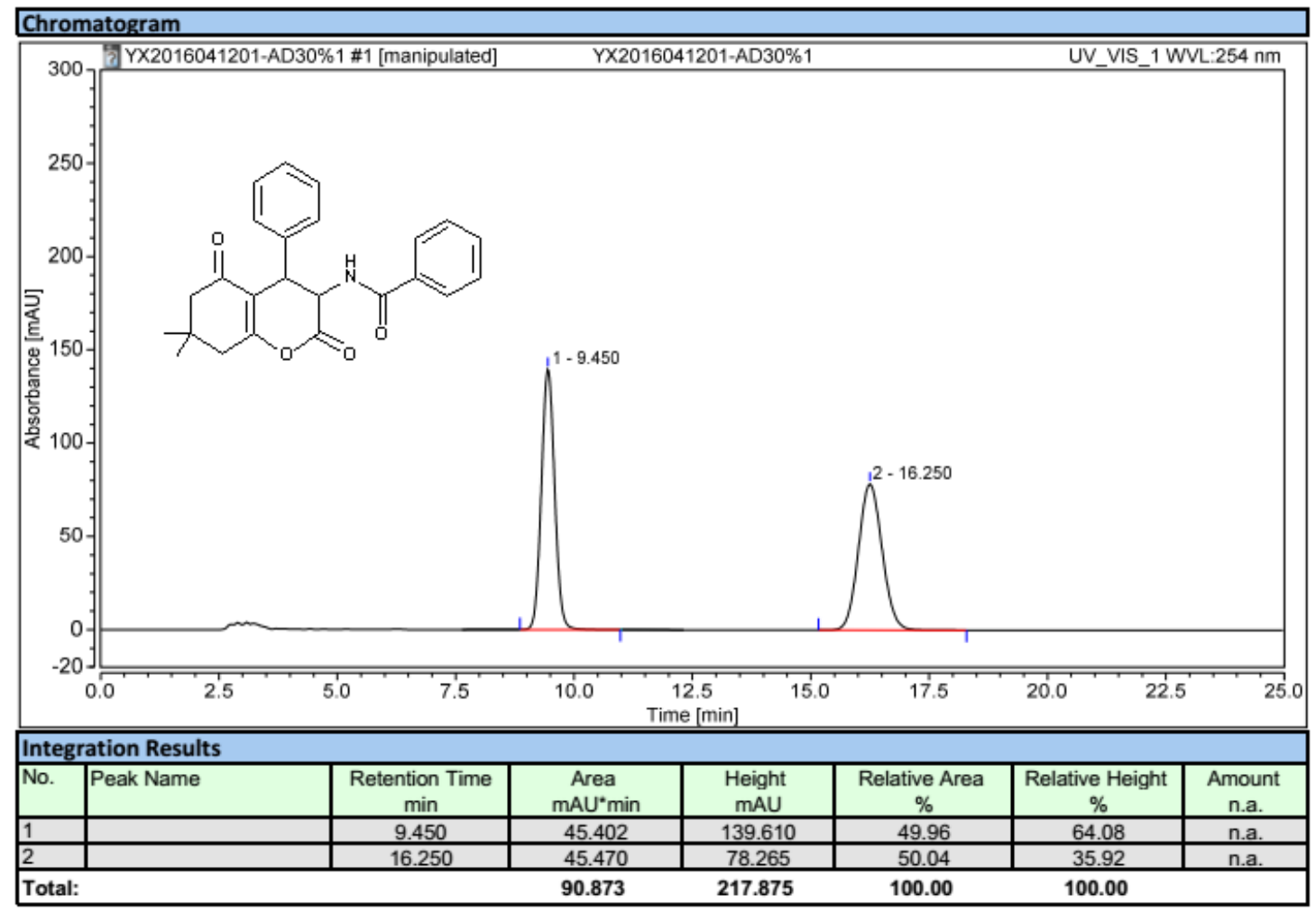

Enantioselective product:

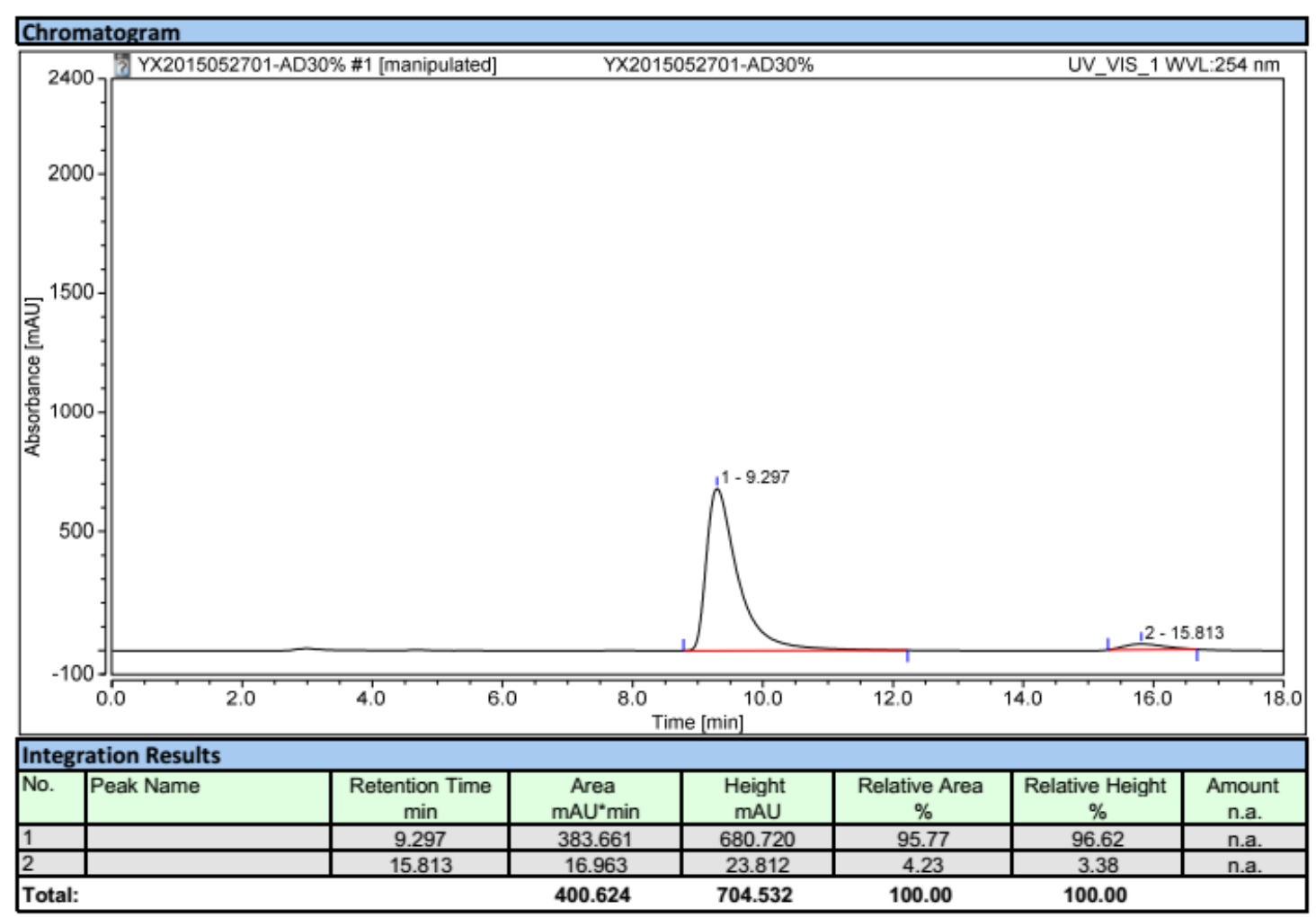


Enantioselective product after recrystallization (generated from ent-3aa):

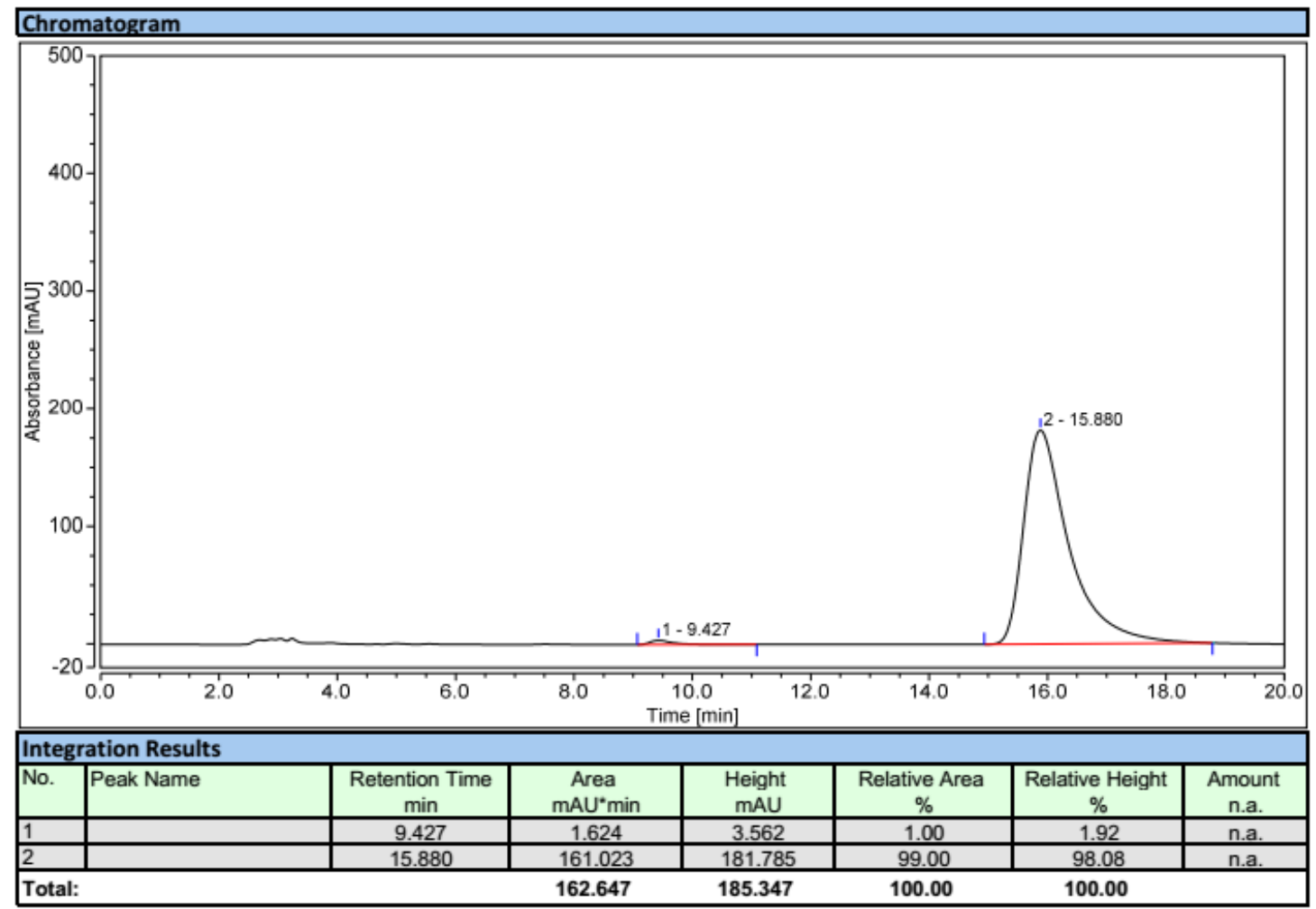




\section{3ba}

Racemic product:

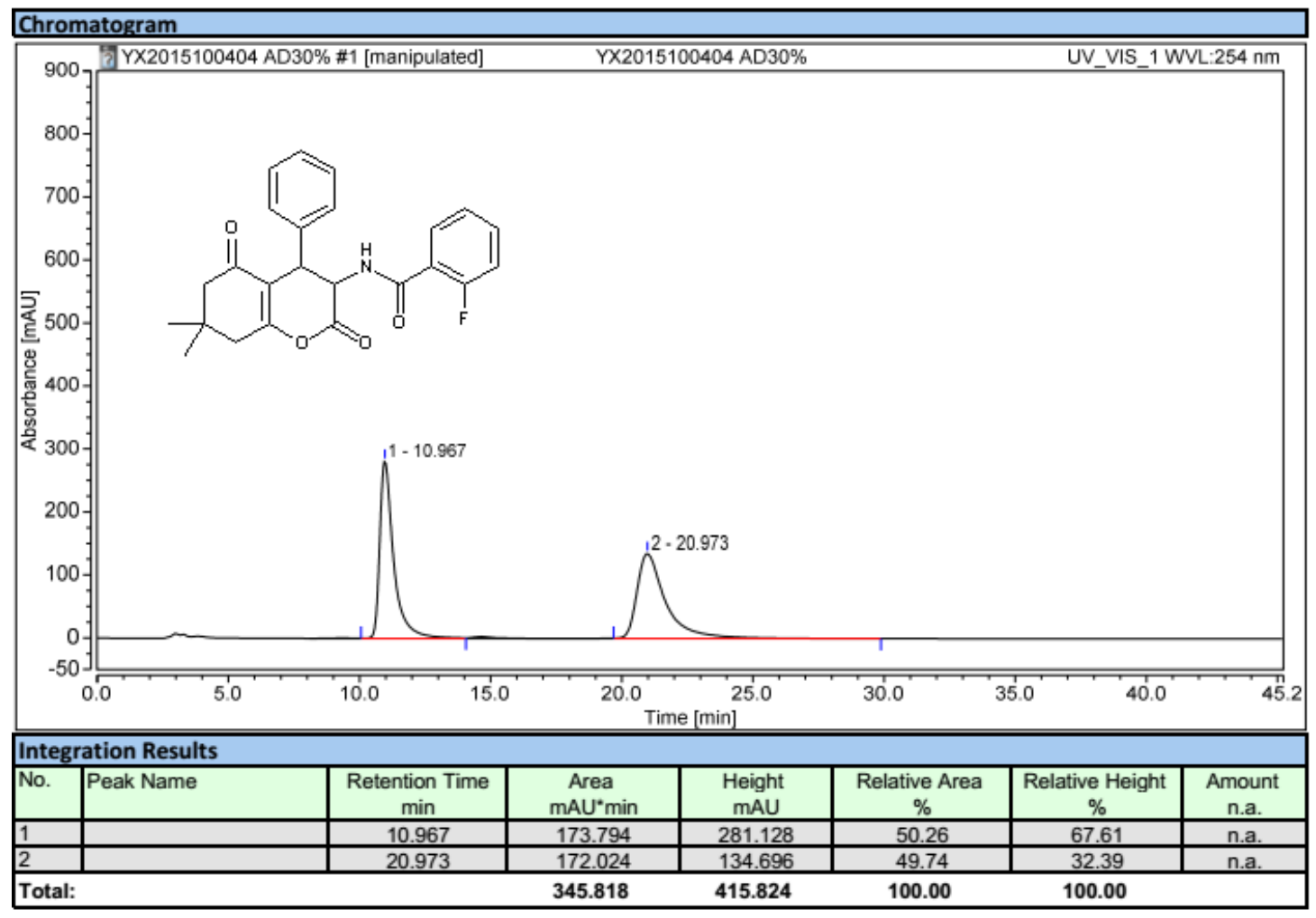

Enantioselective product:

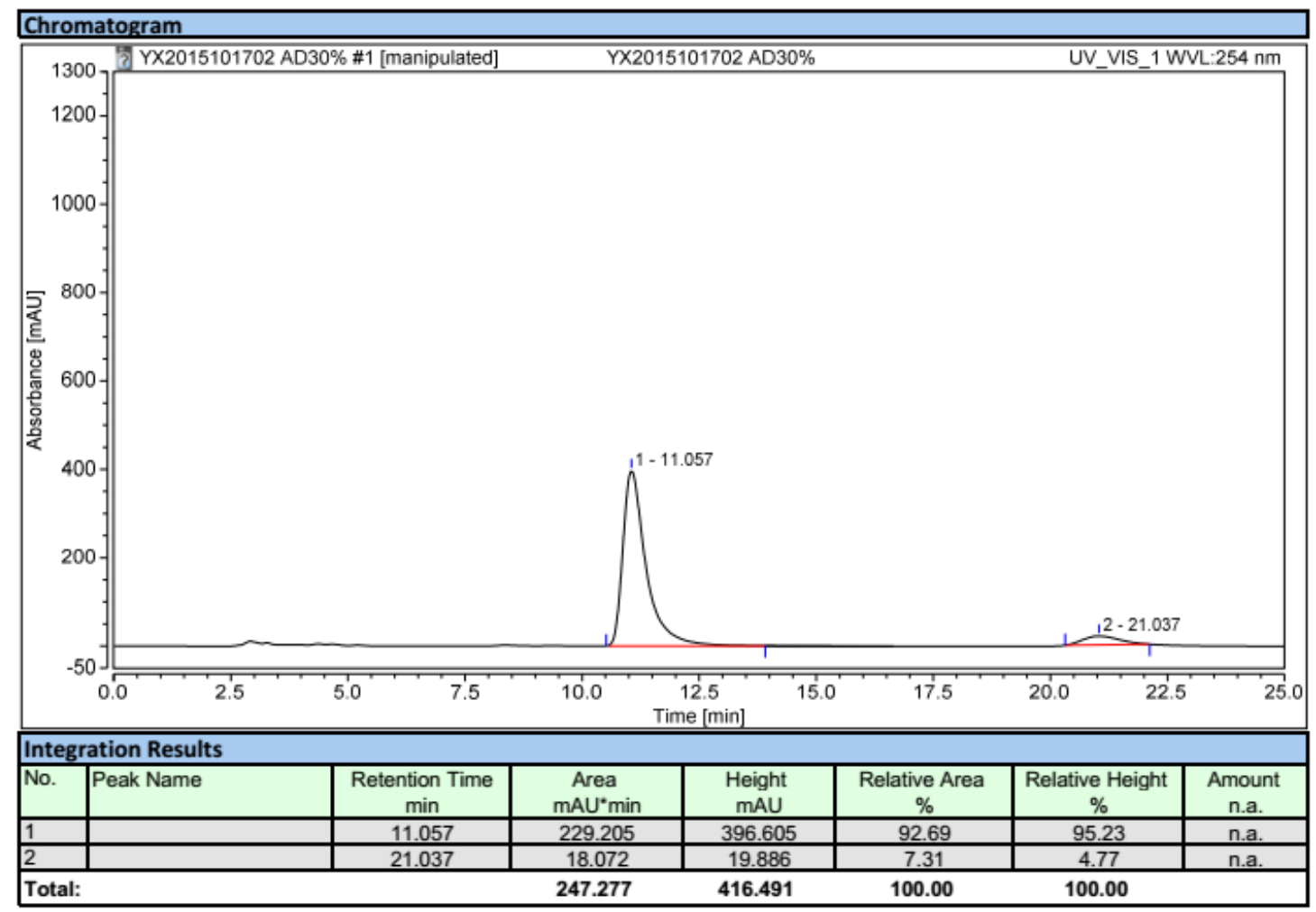




\section{3ca}

Racemic product:

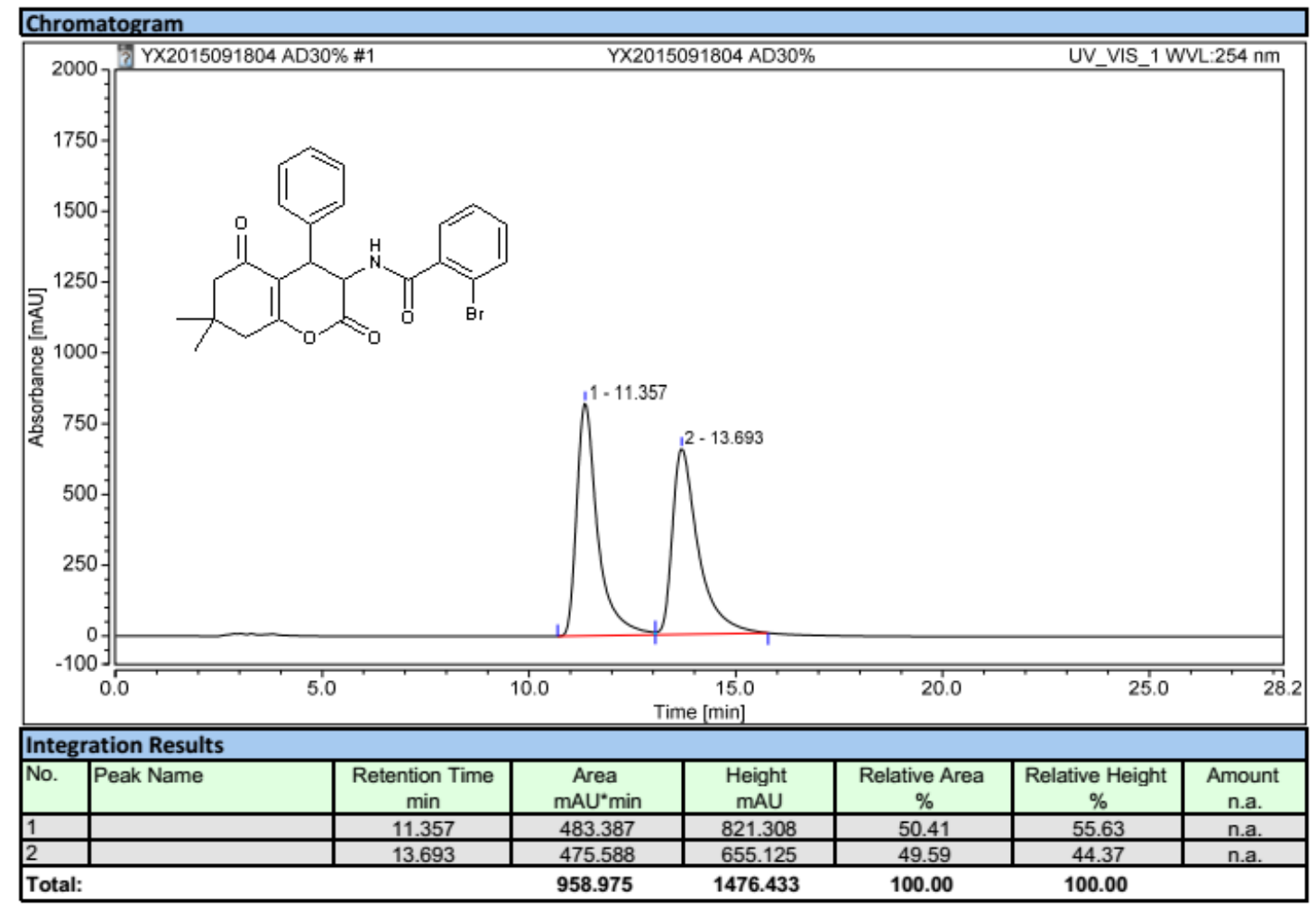

Enantioselective product:

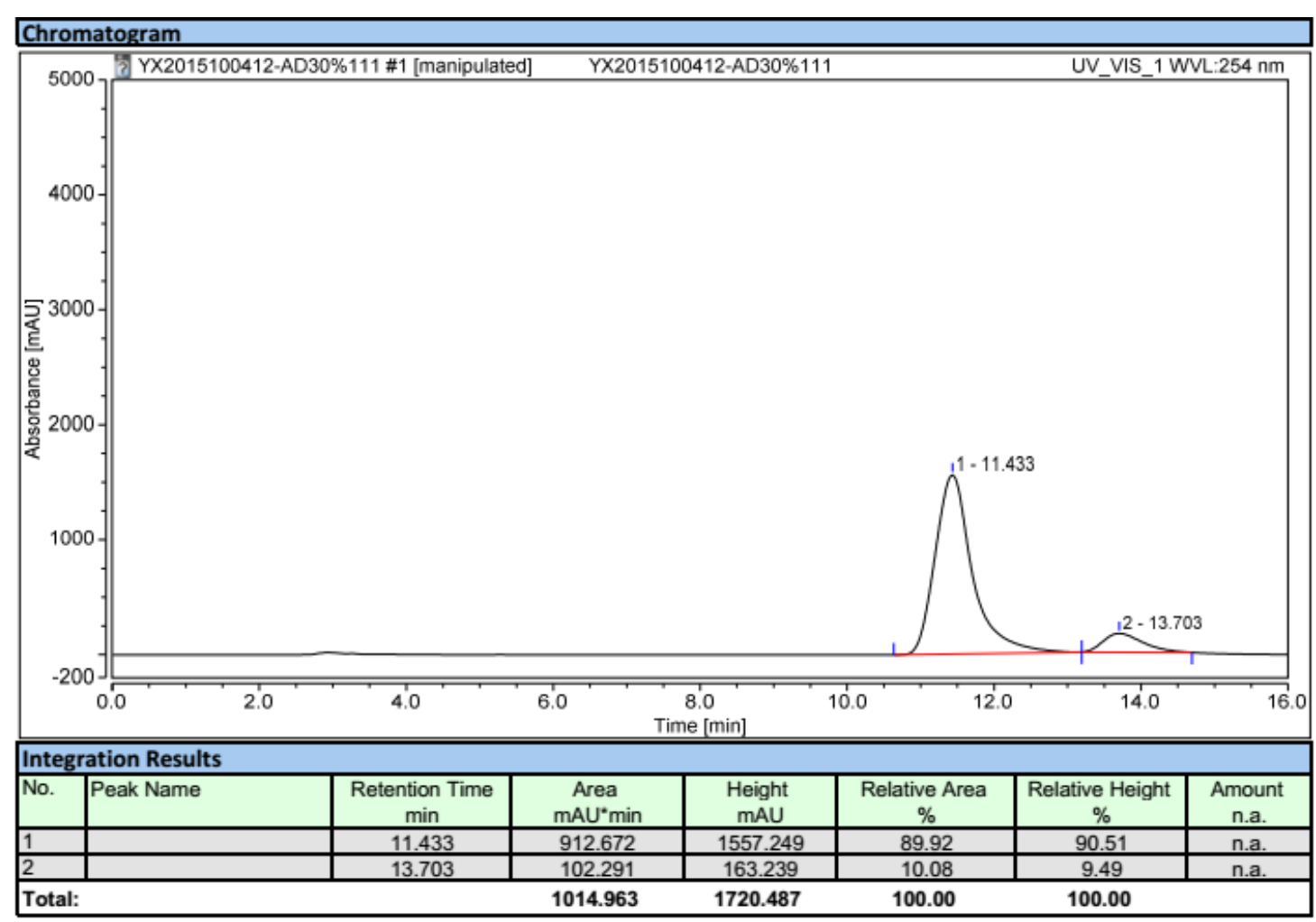


Enantioselective product after recrystallization:

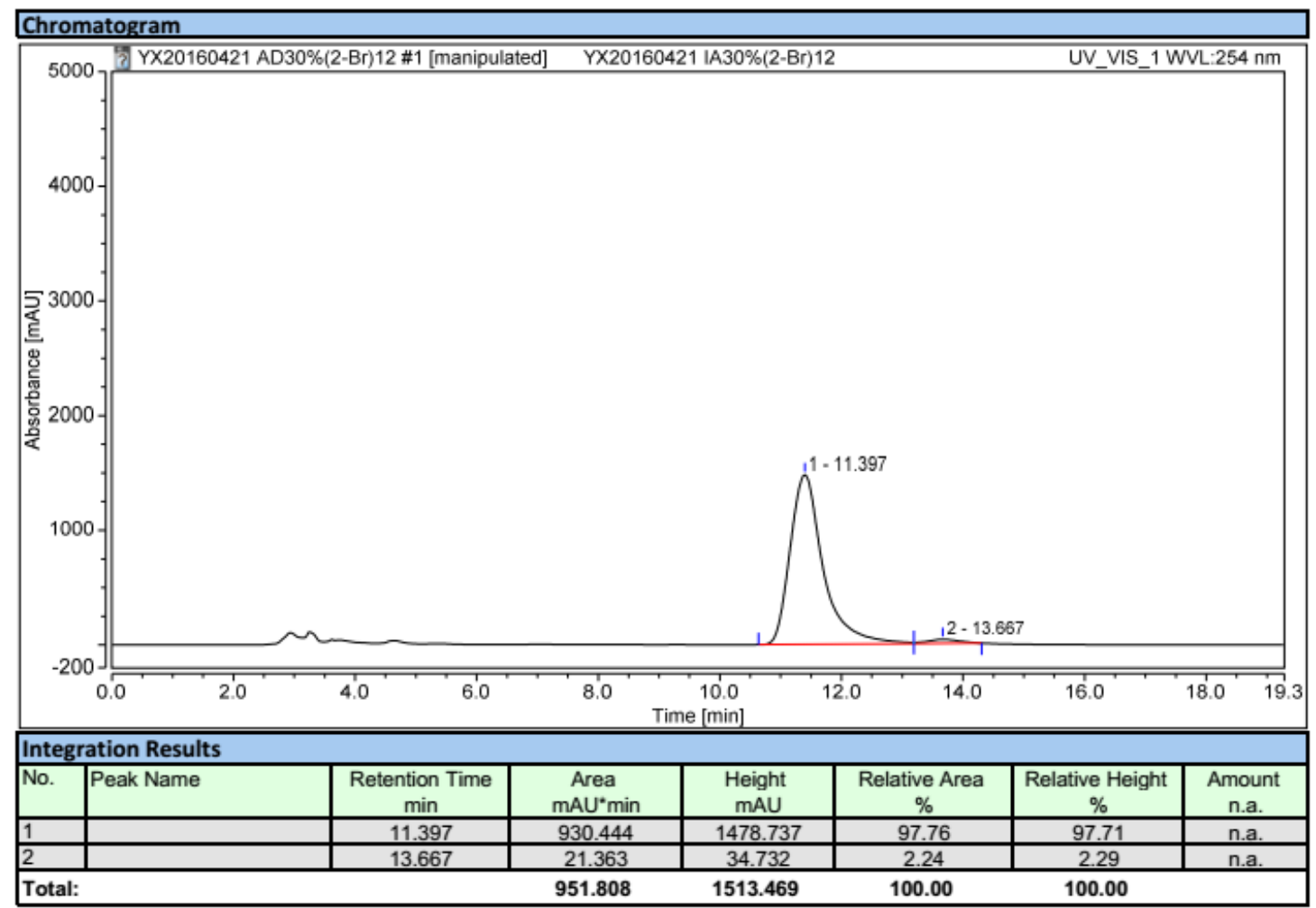




\section{3da}

Racemic product:

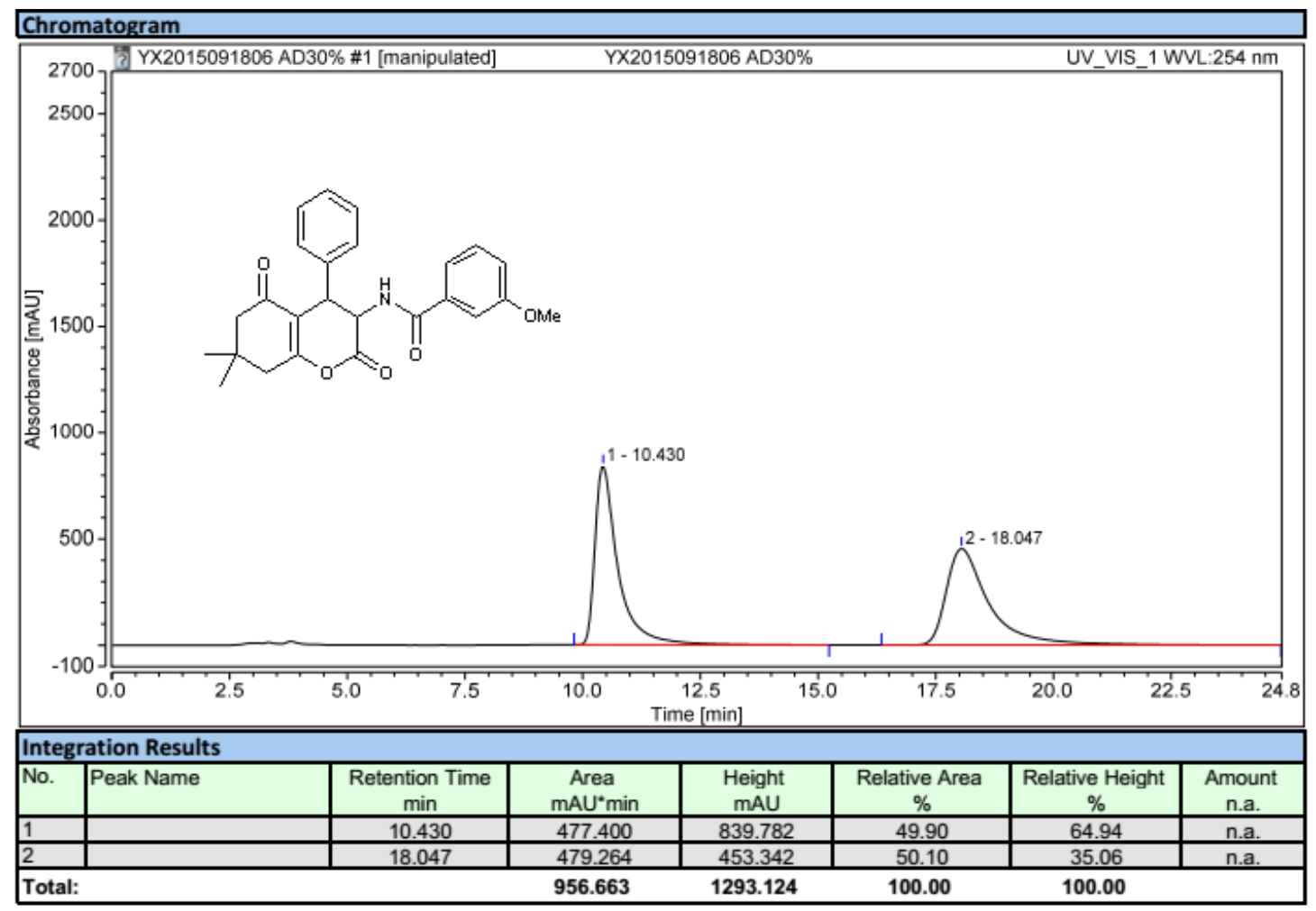

Enantioselective product:

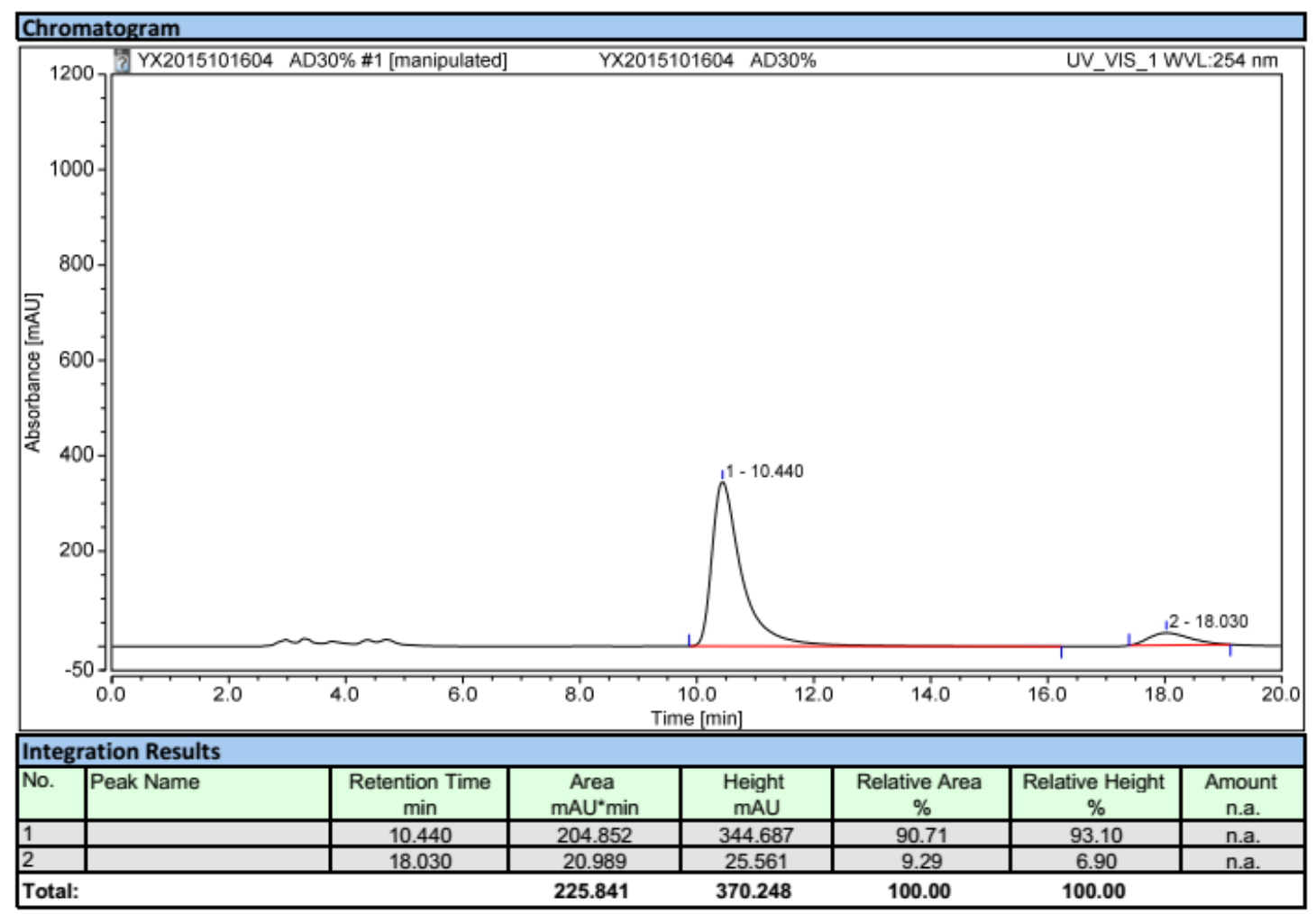


Enantioselective product after recrystallization:

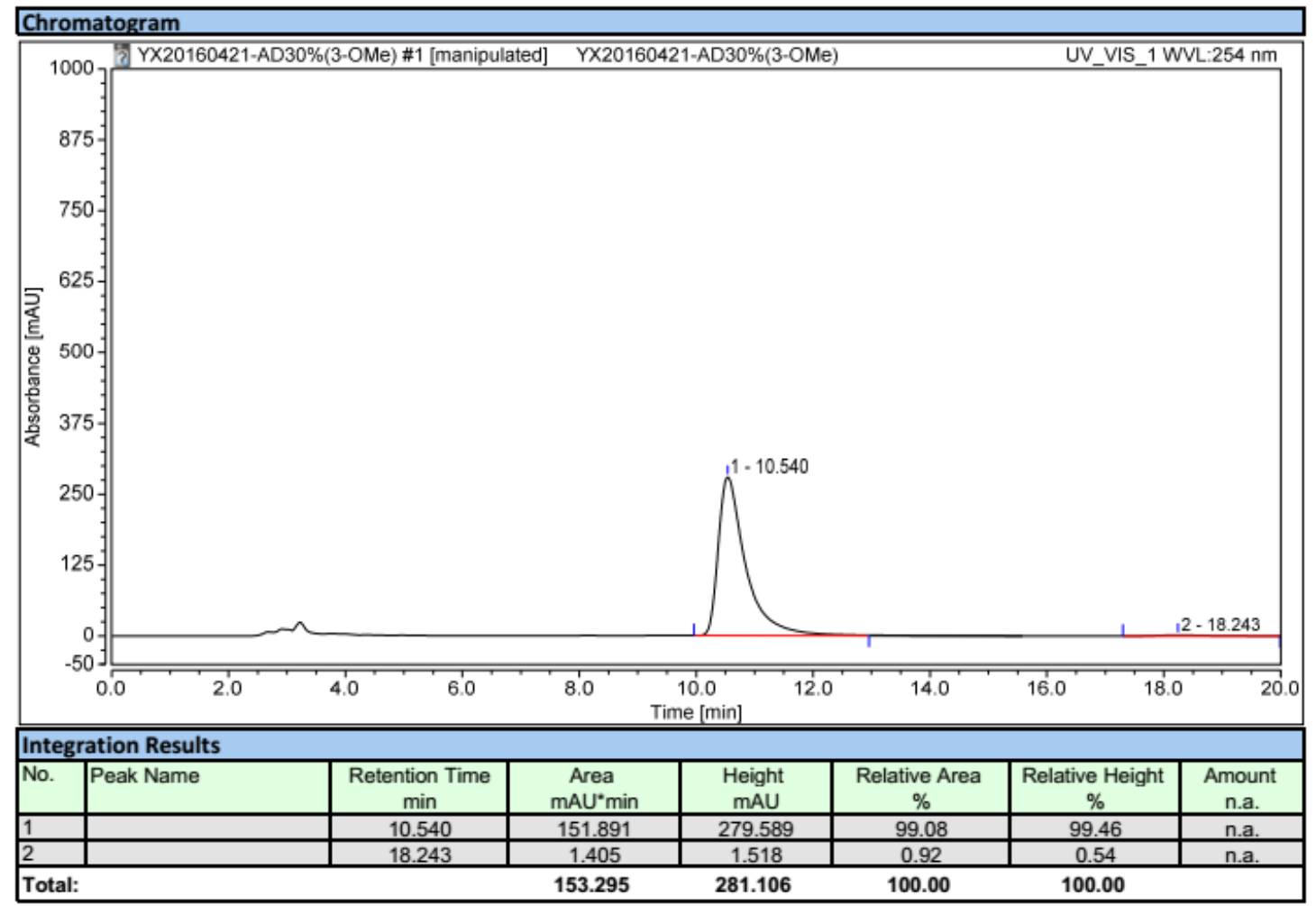




\section{3ea}

Racemic product:

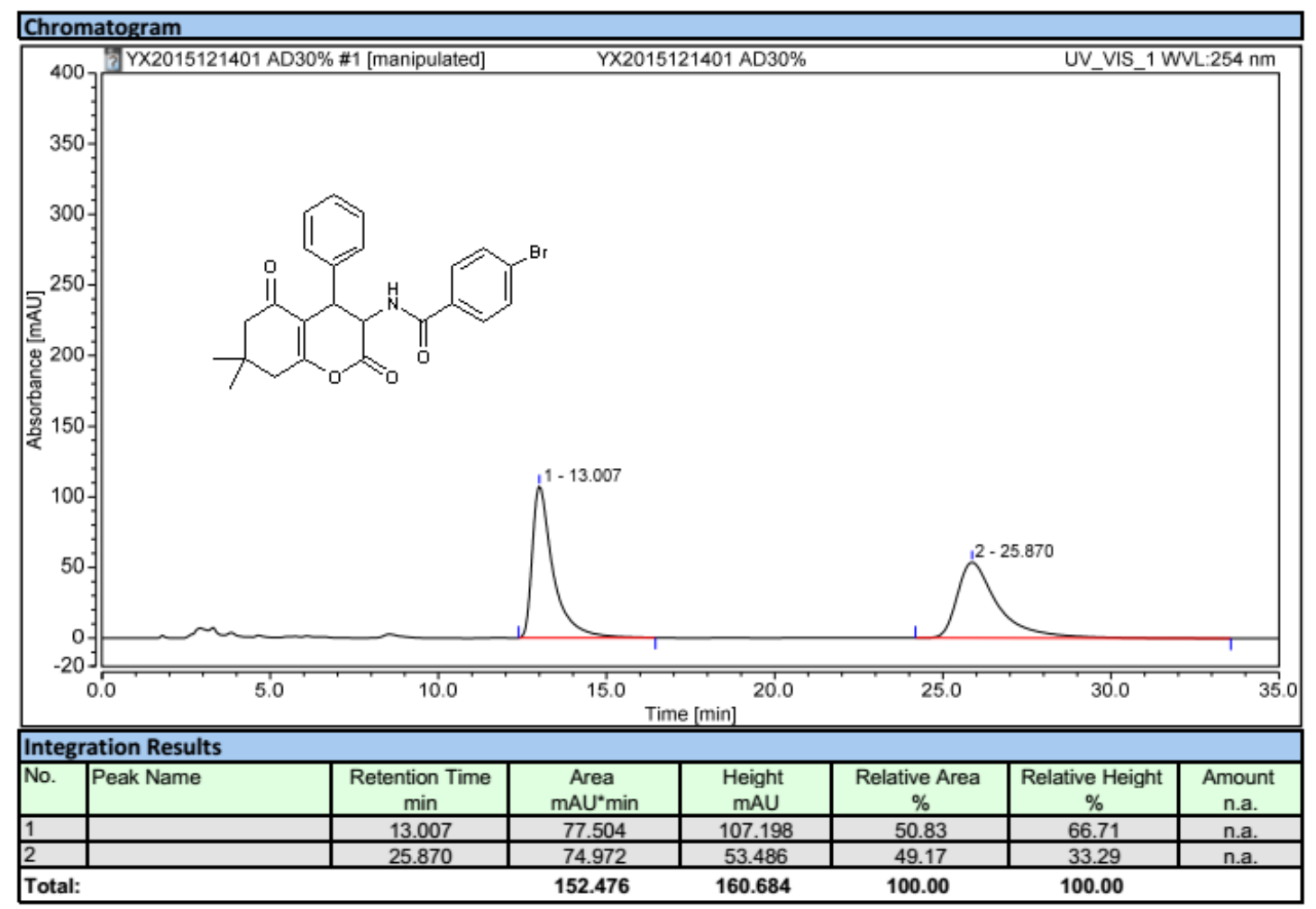

Enantioselective product:

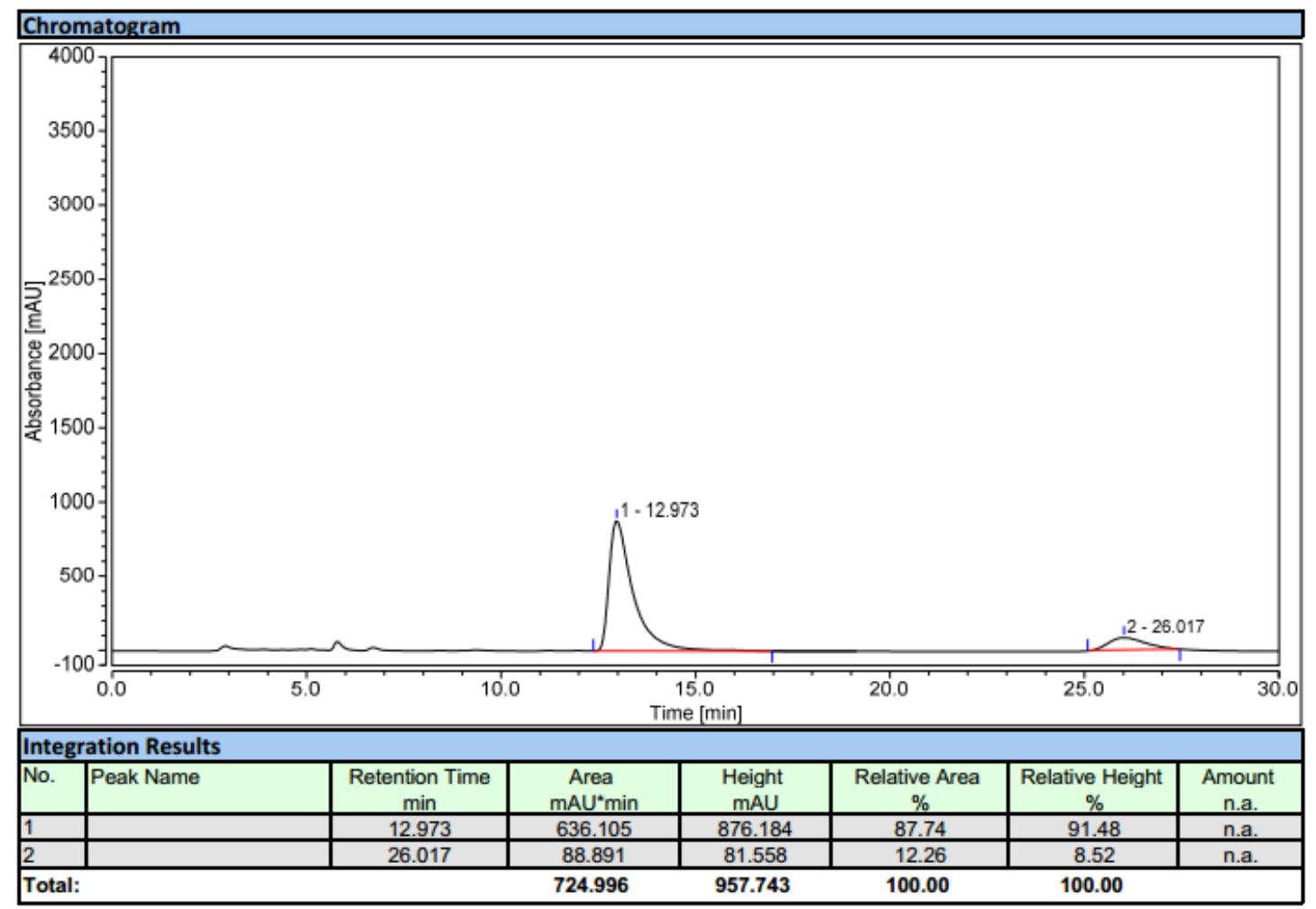




\section{3fa}

Racemic product:

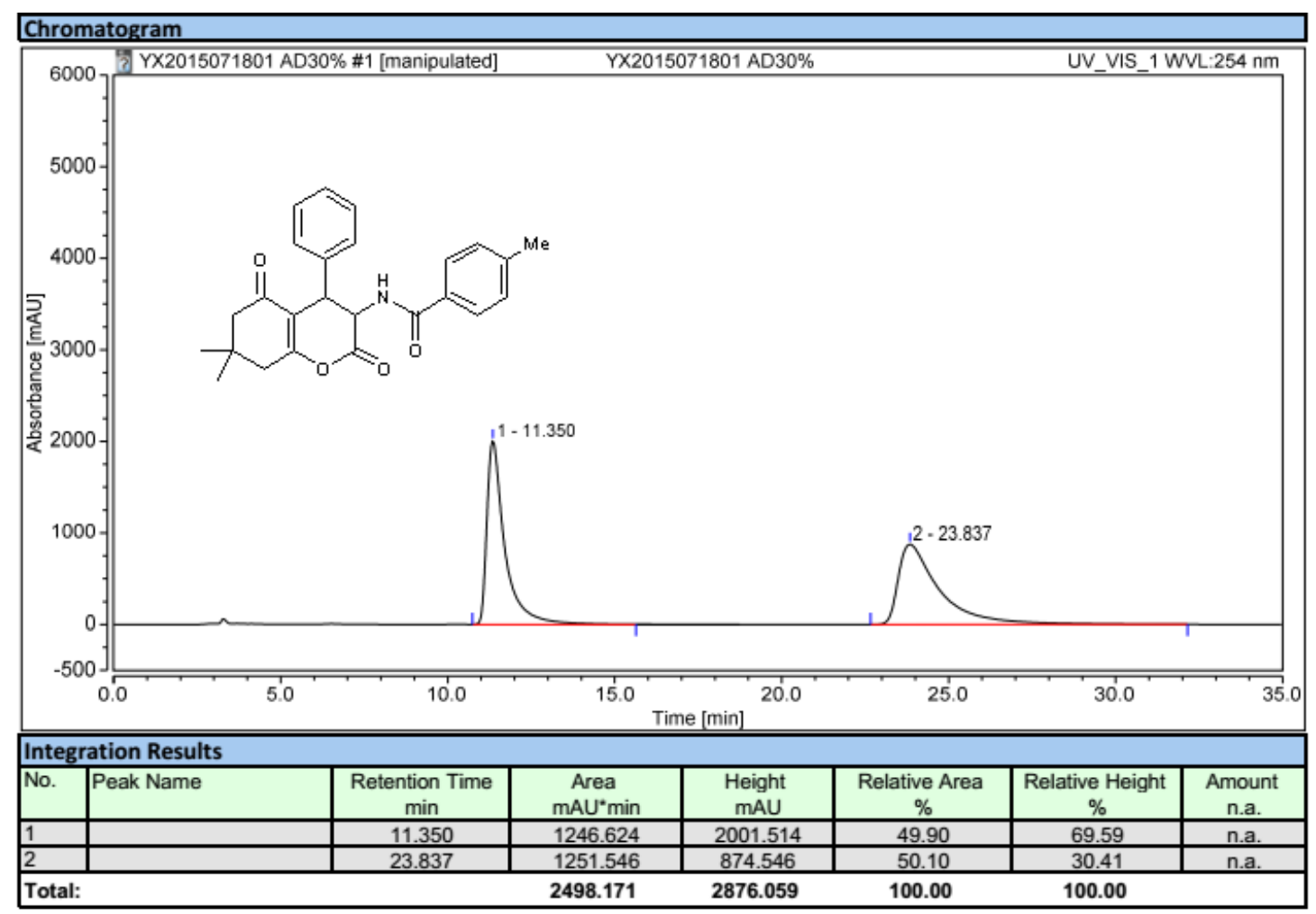

Enantioselective product:

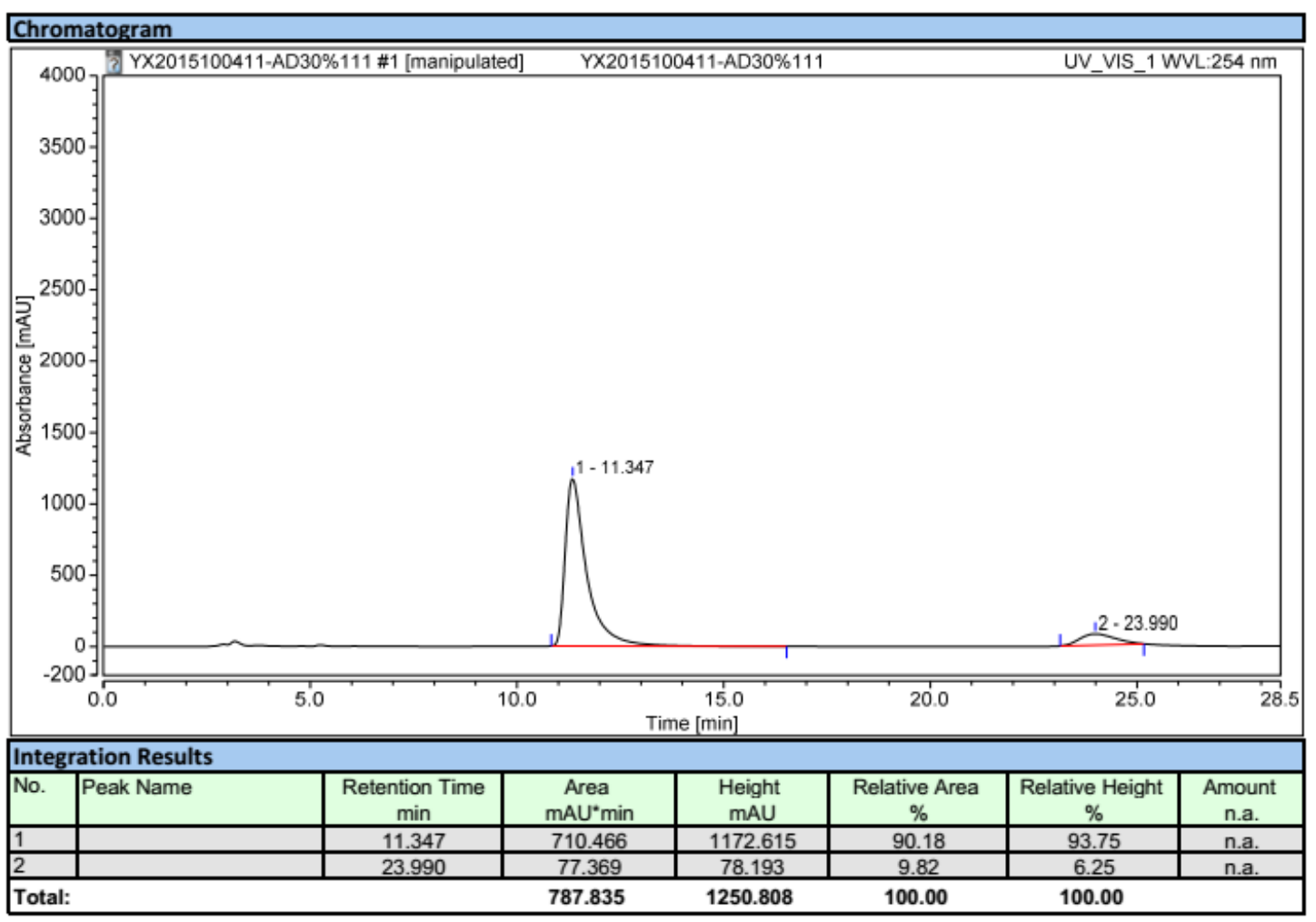


Enantioselective product after recrystallization:

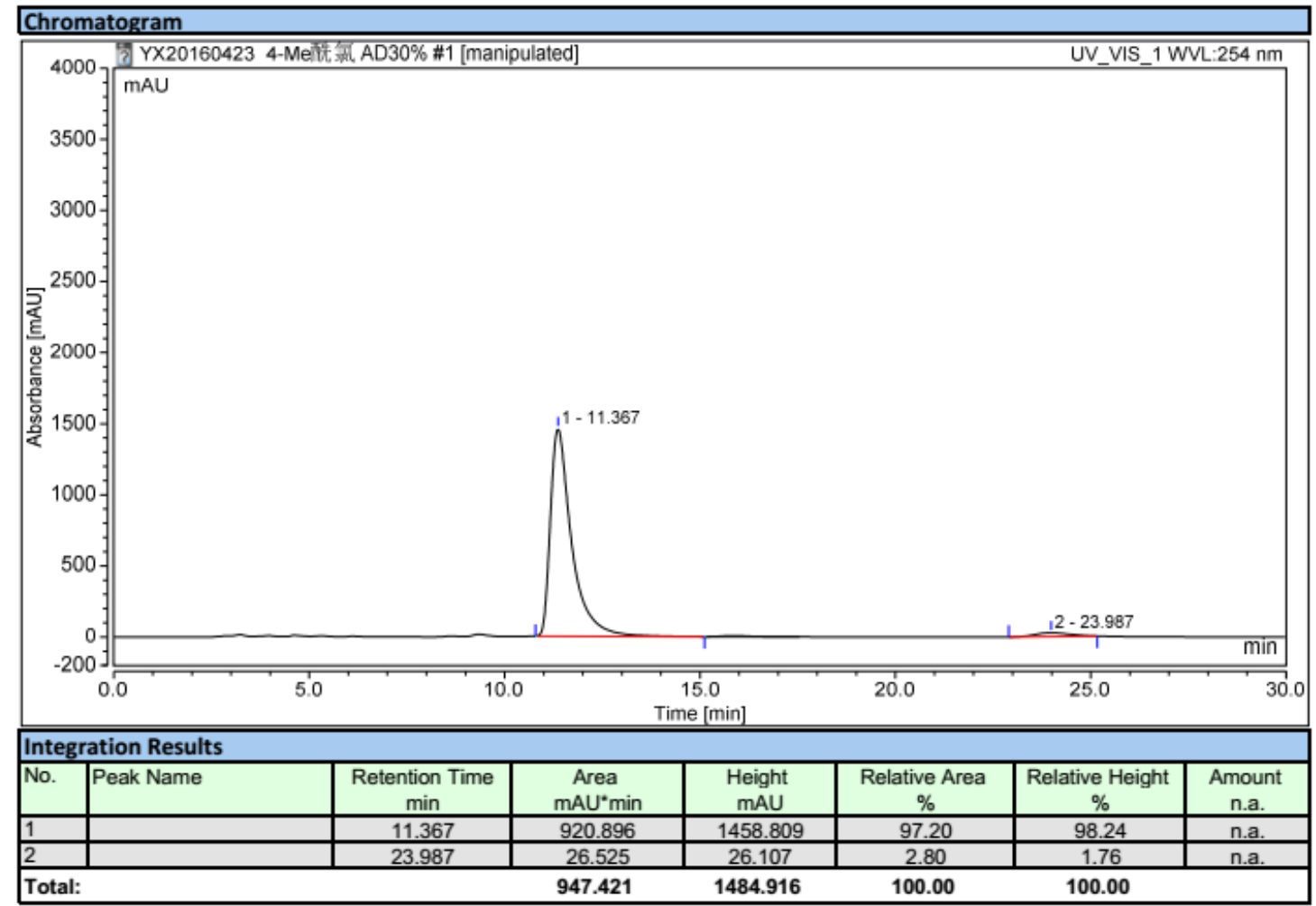




\section{3ga}

Racemic product:

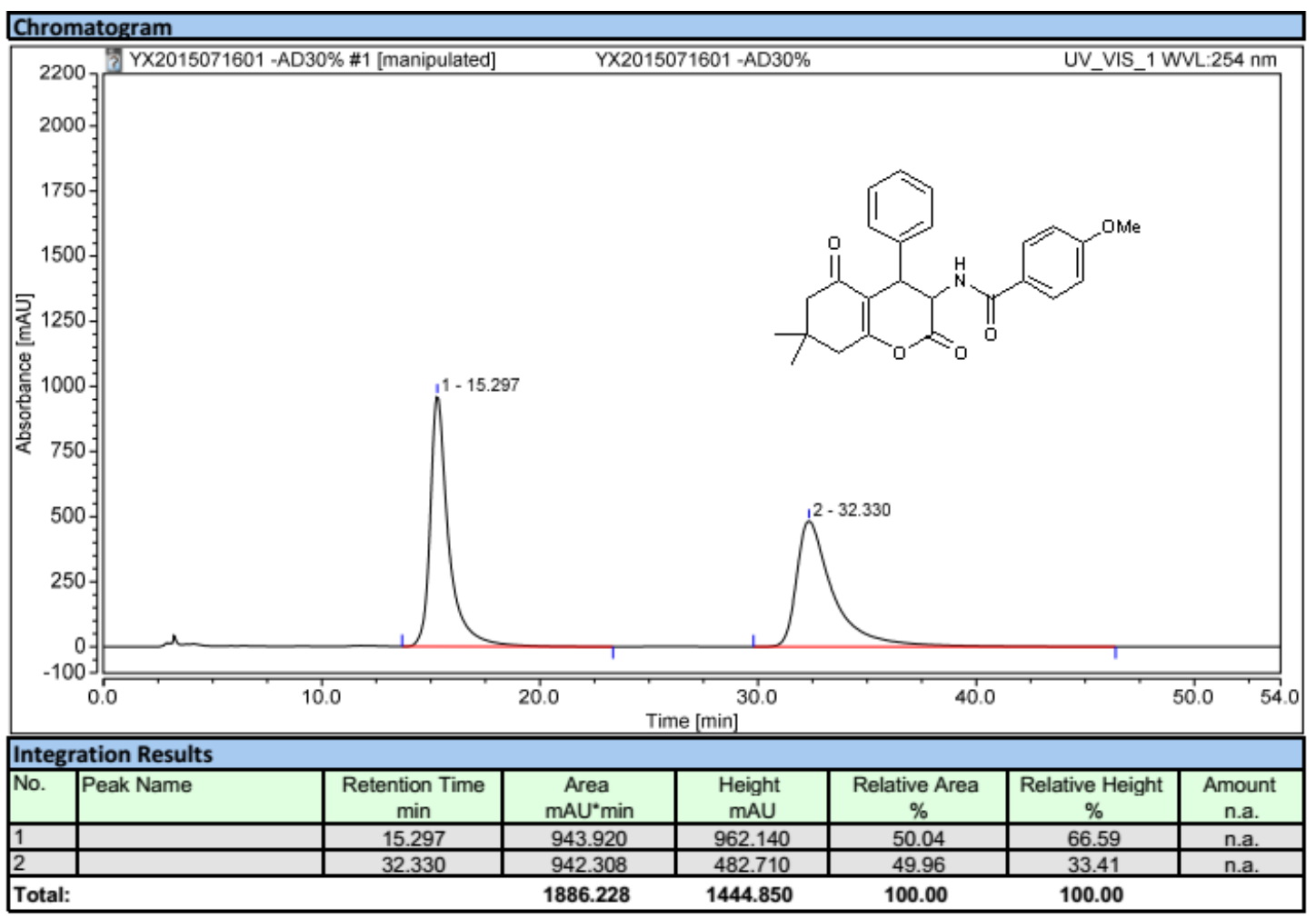

Enantioselective product:

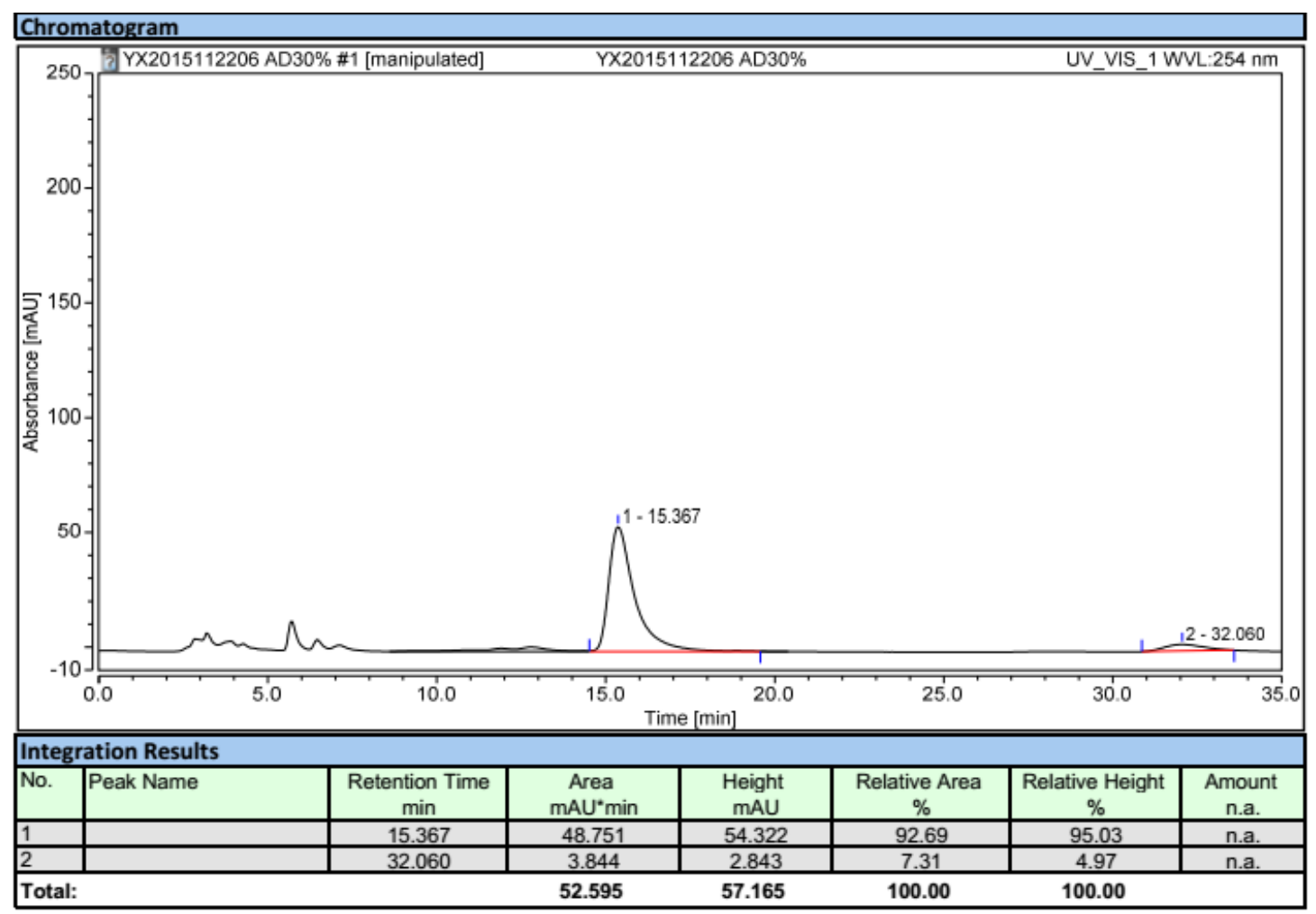




\section{3ha}

Racemic product:

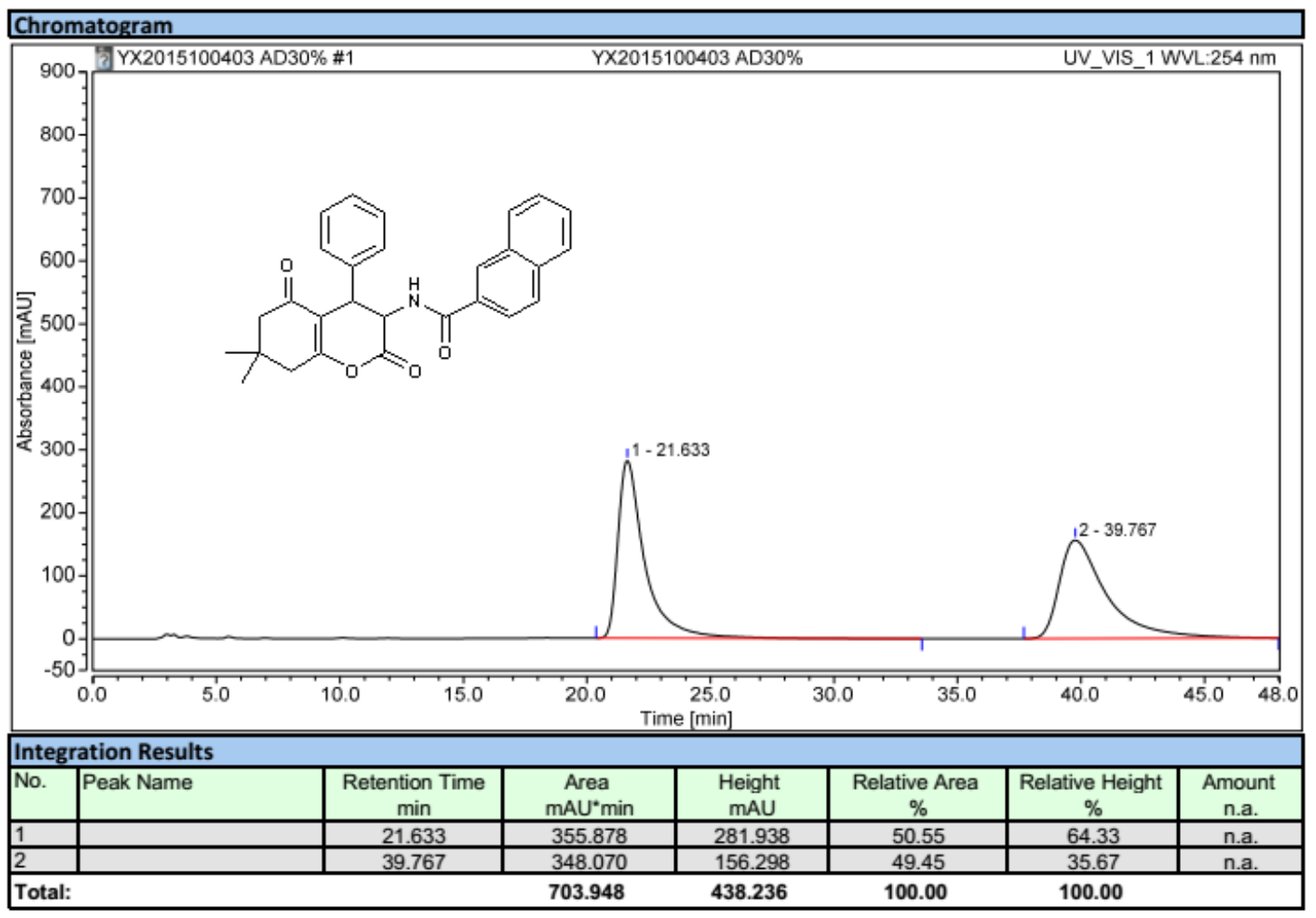

Enantioselective product:

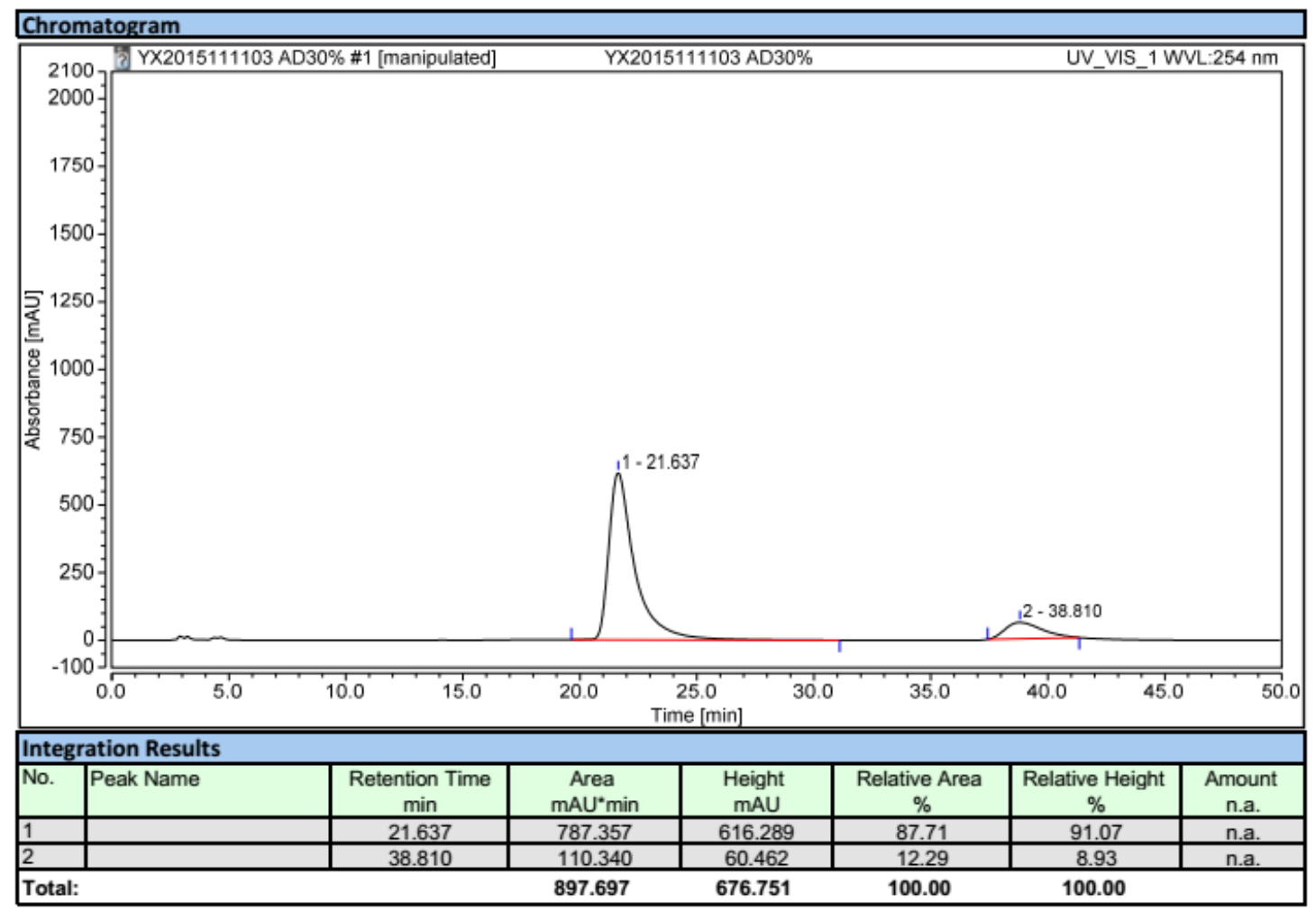


Enantioselective product after recrystallization:

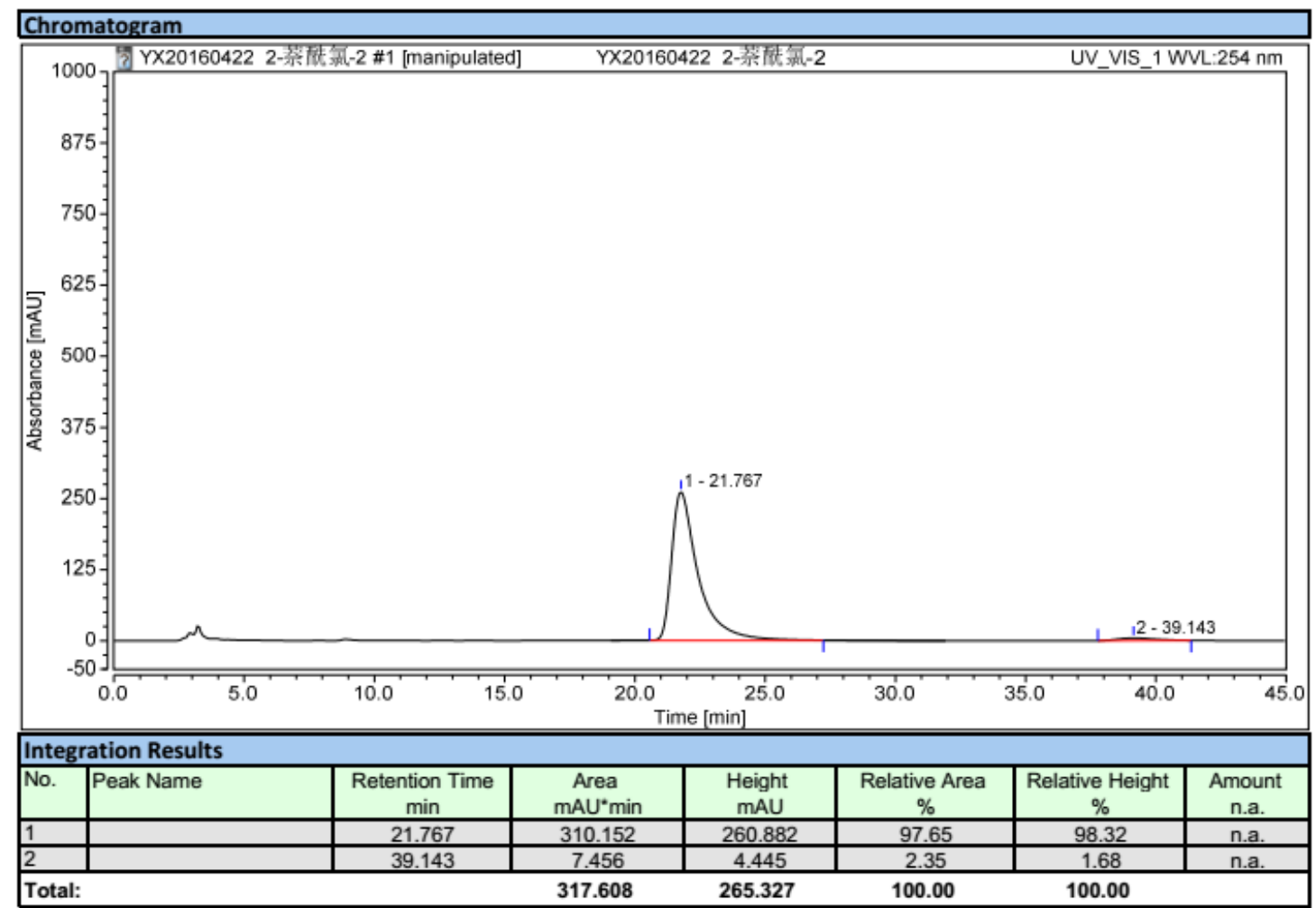




\section{3ia}

Racemic product:

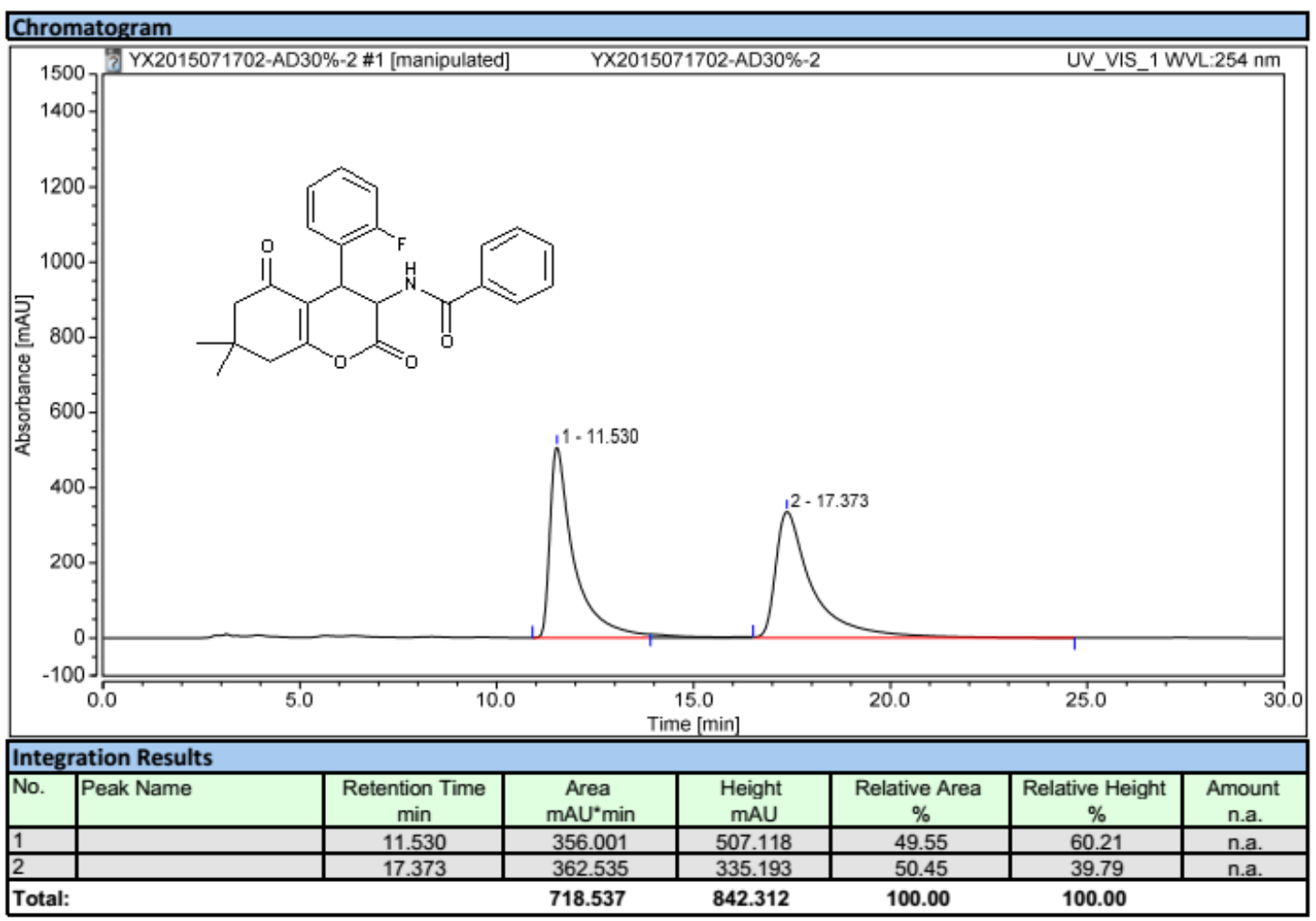

Enantioselective product:

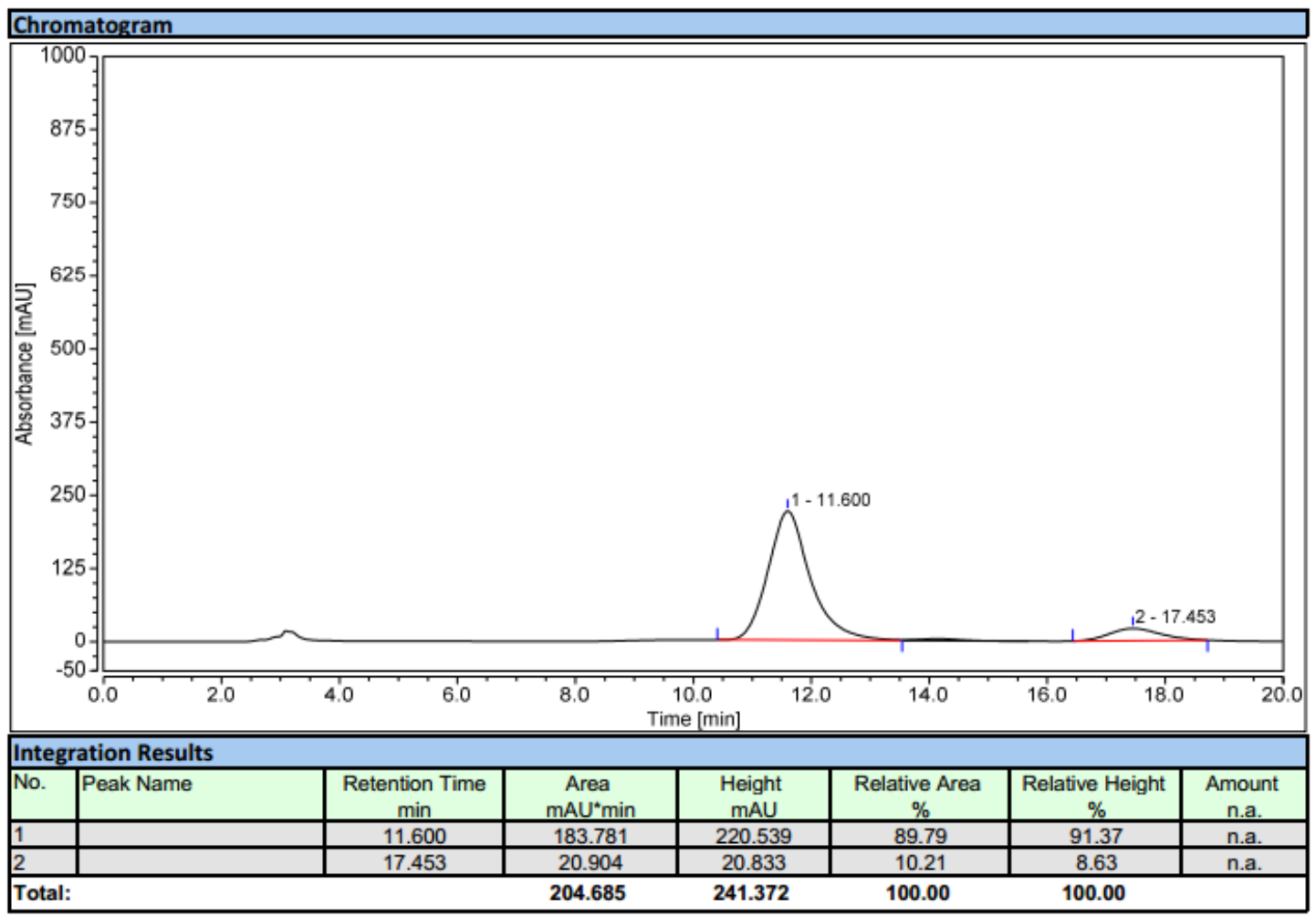


Enantioselective product after recrystallization:

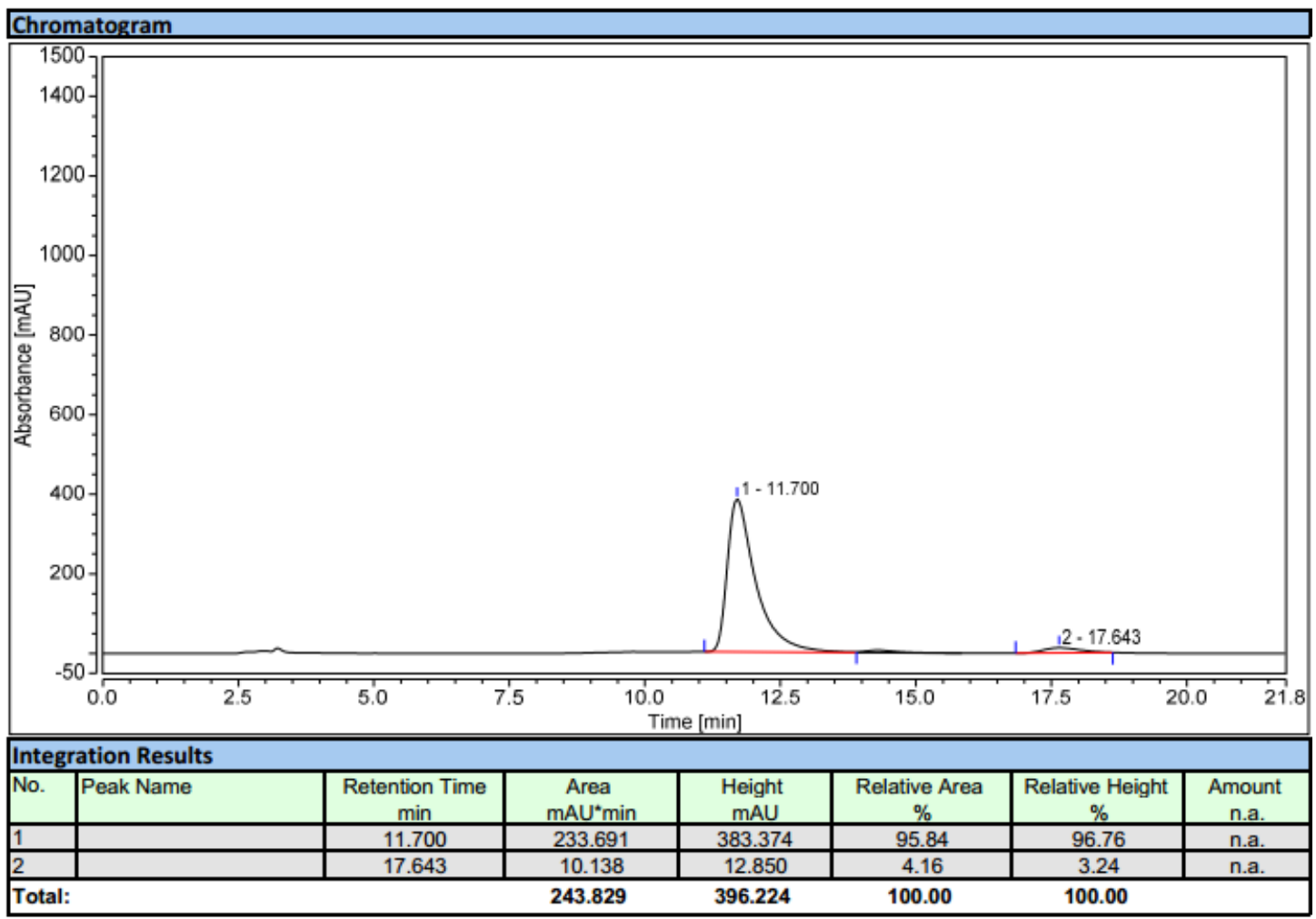




\section{3ja}

Racemic product:

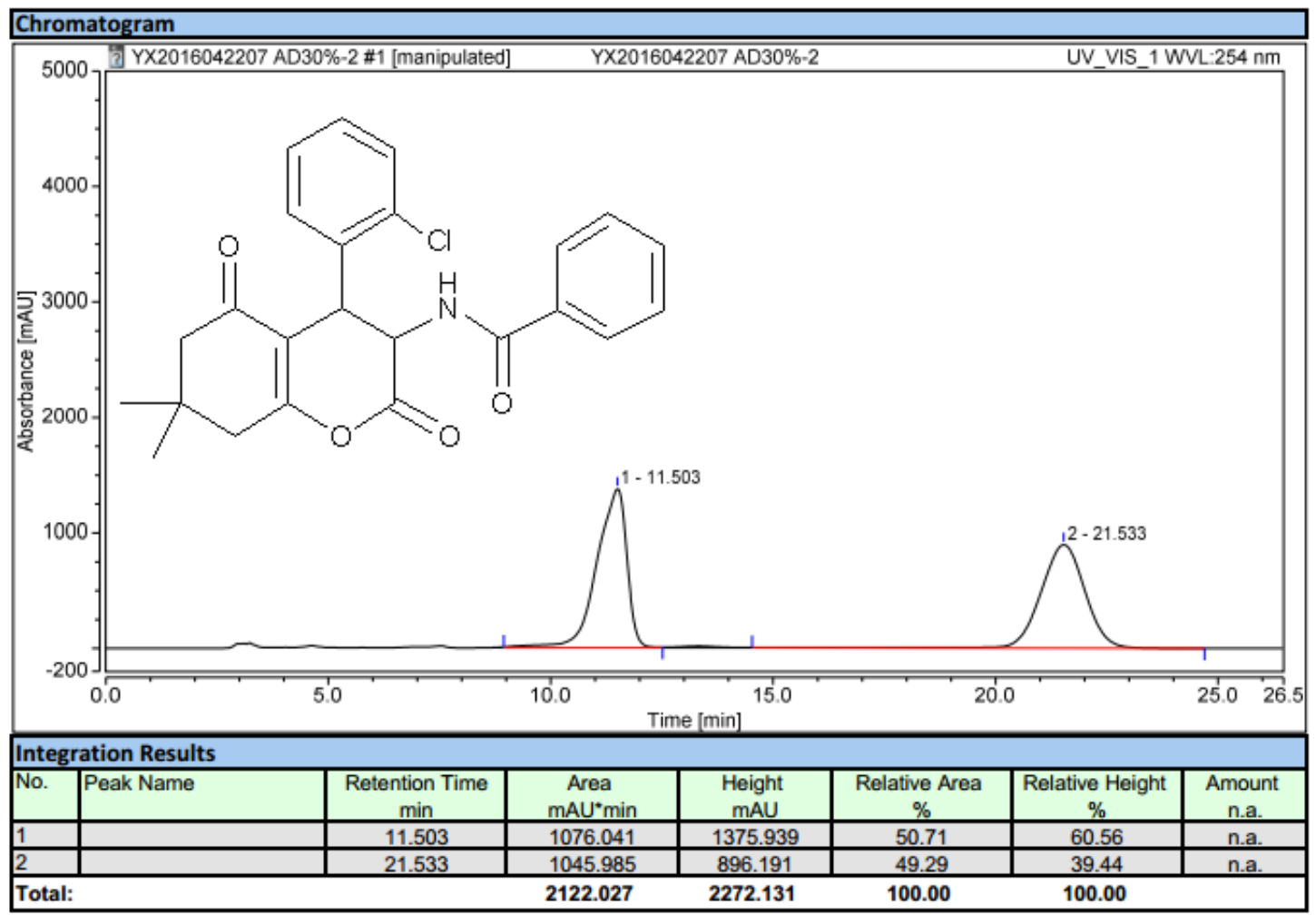

Enantioselective product:

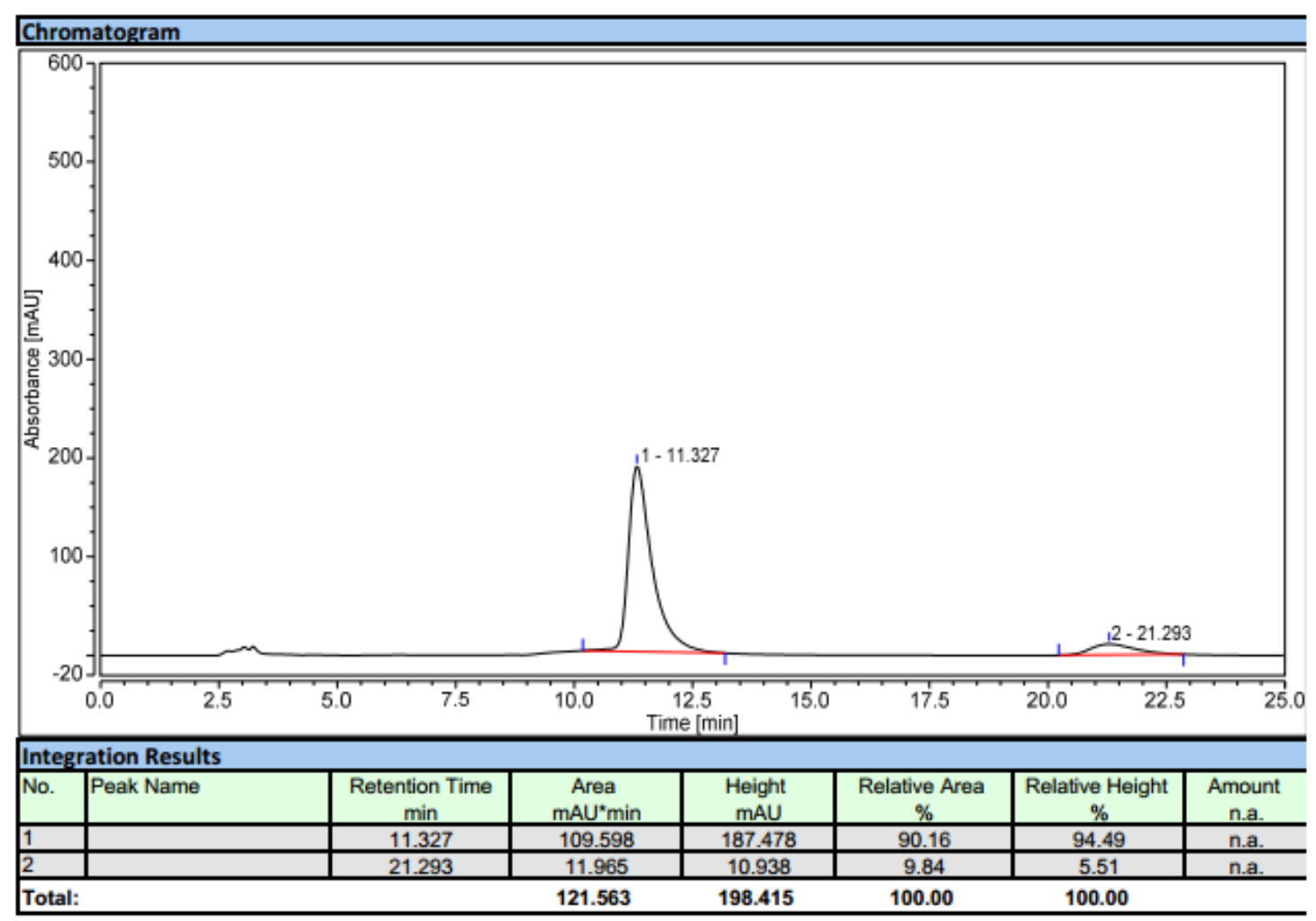


Enantioselective product after recrystallization:

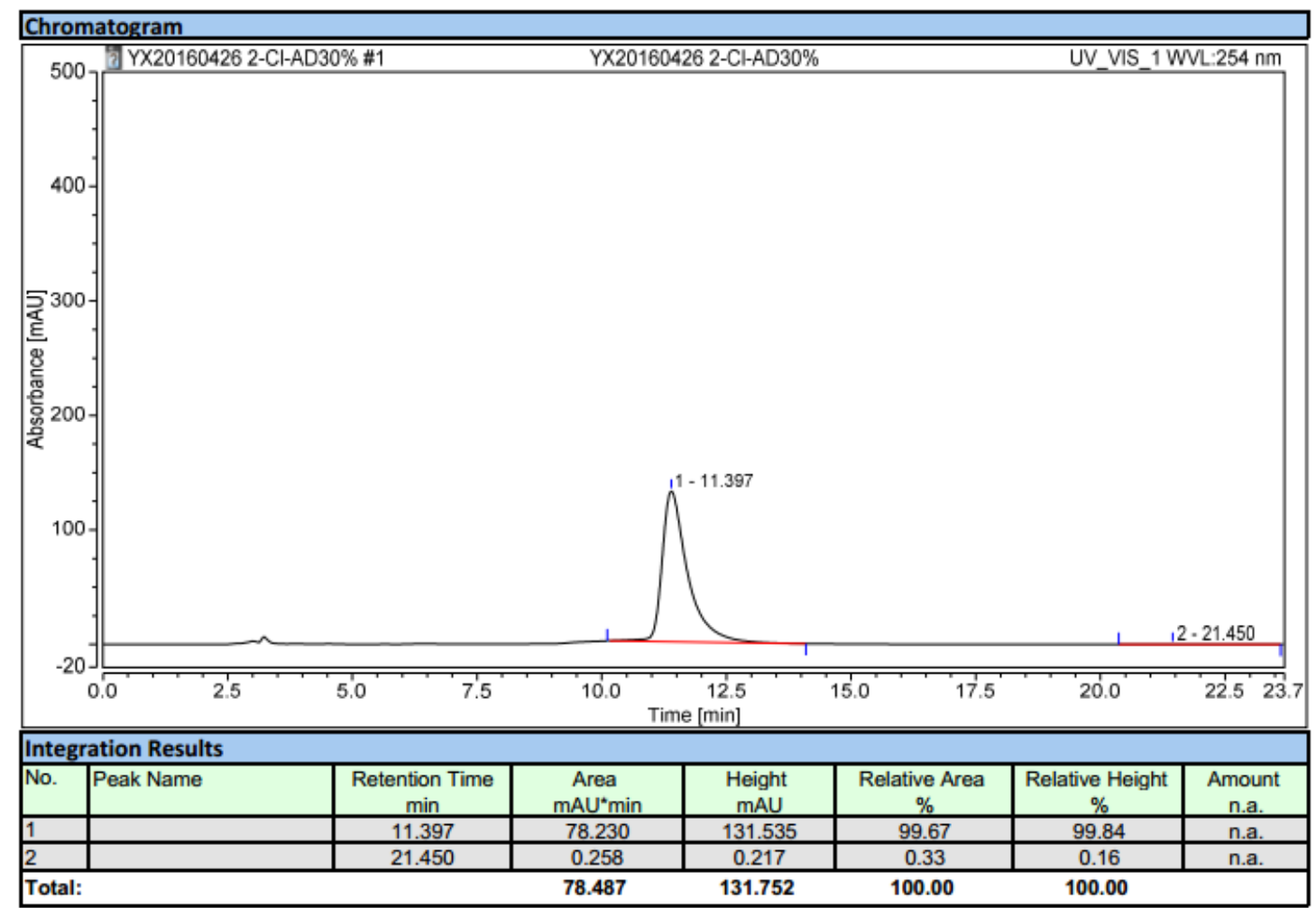




\section{3ka}

Racemic product:

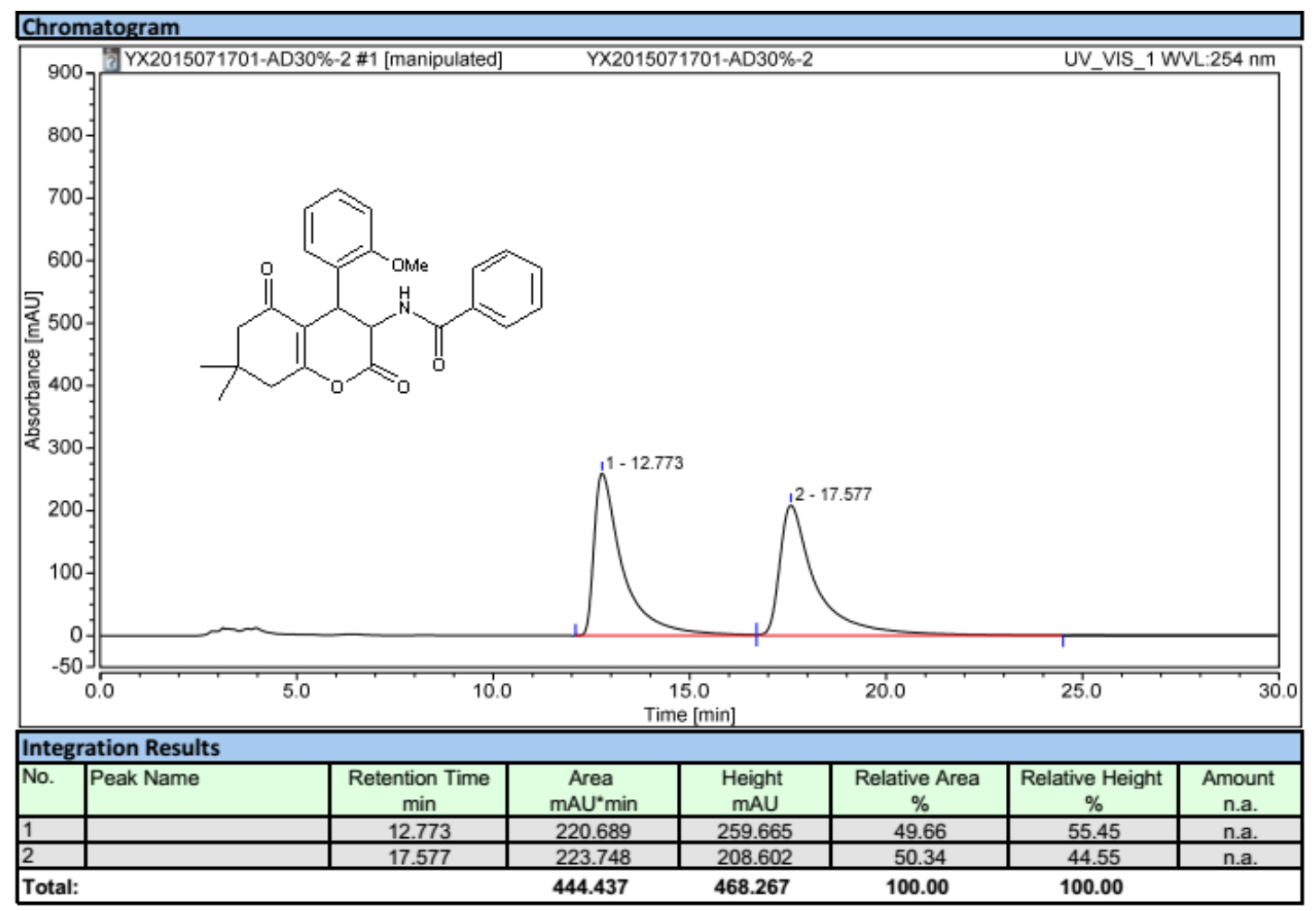

Enantioselective product:

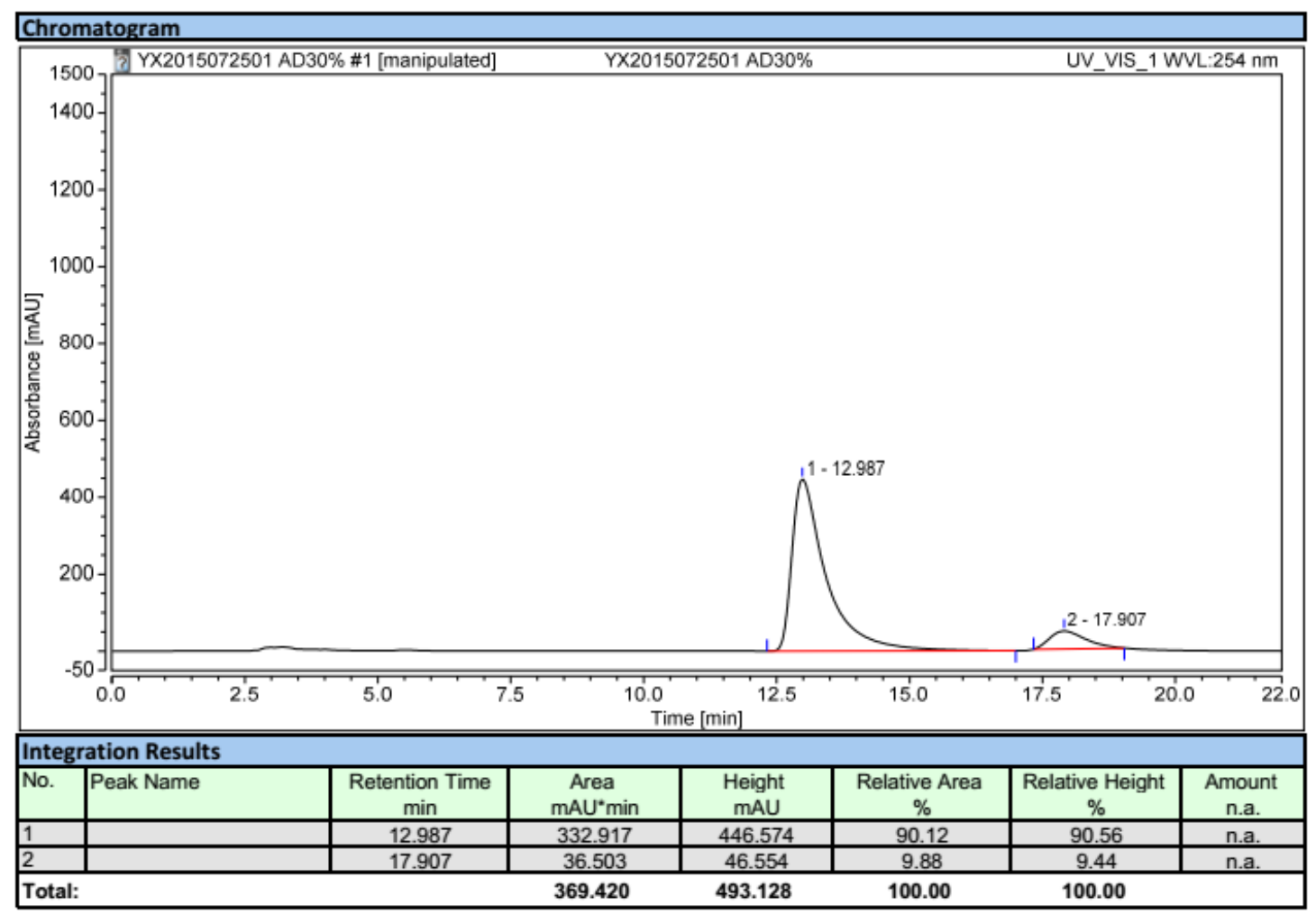


Enantioselective product after recrystallization:

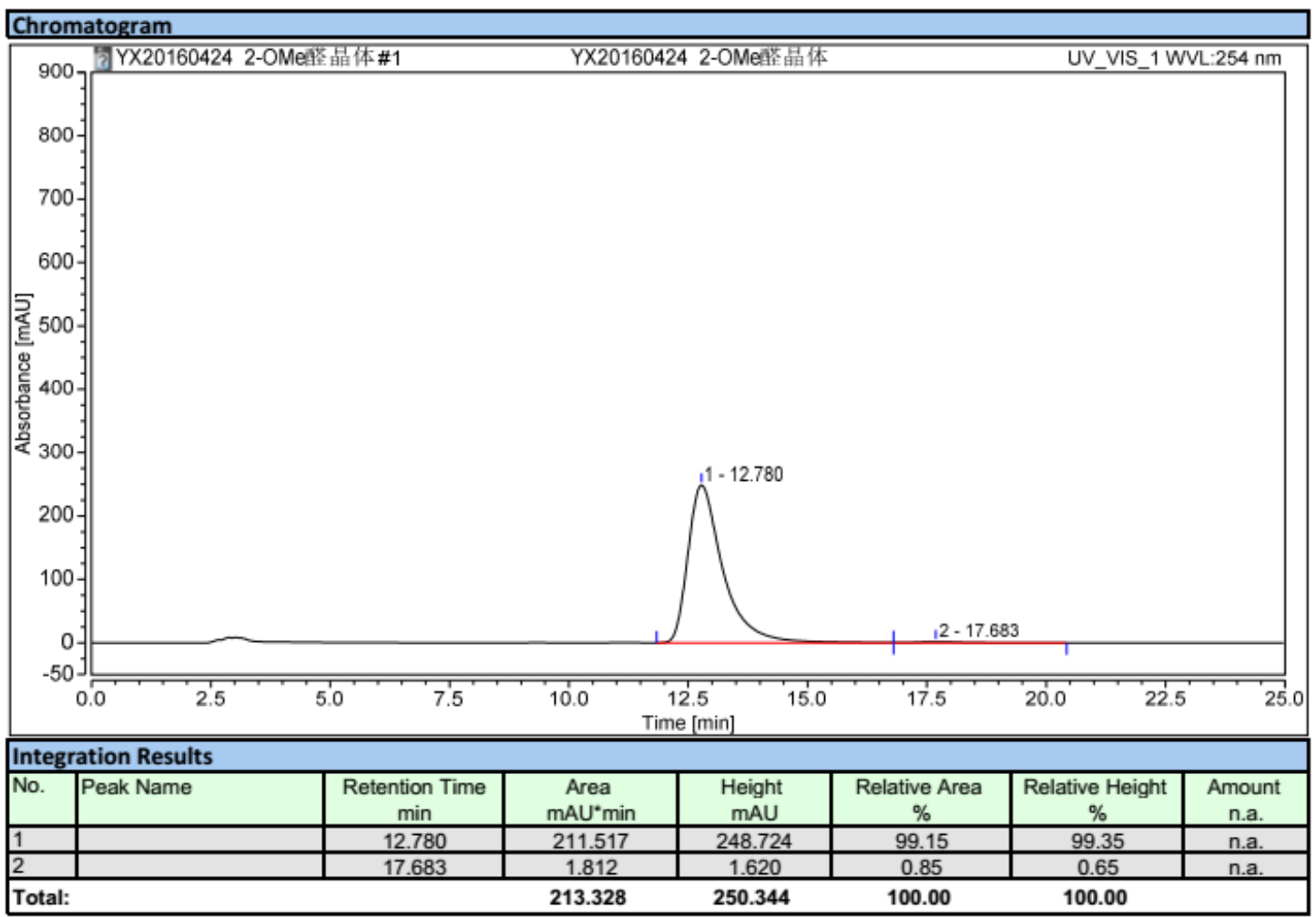




\section{3la}

Racemic product:

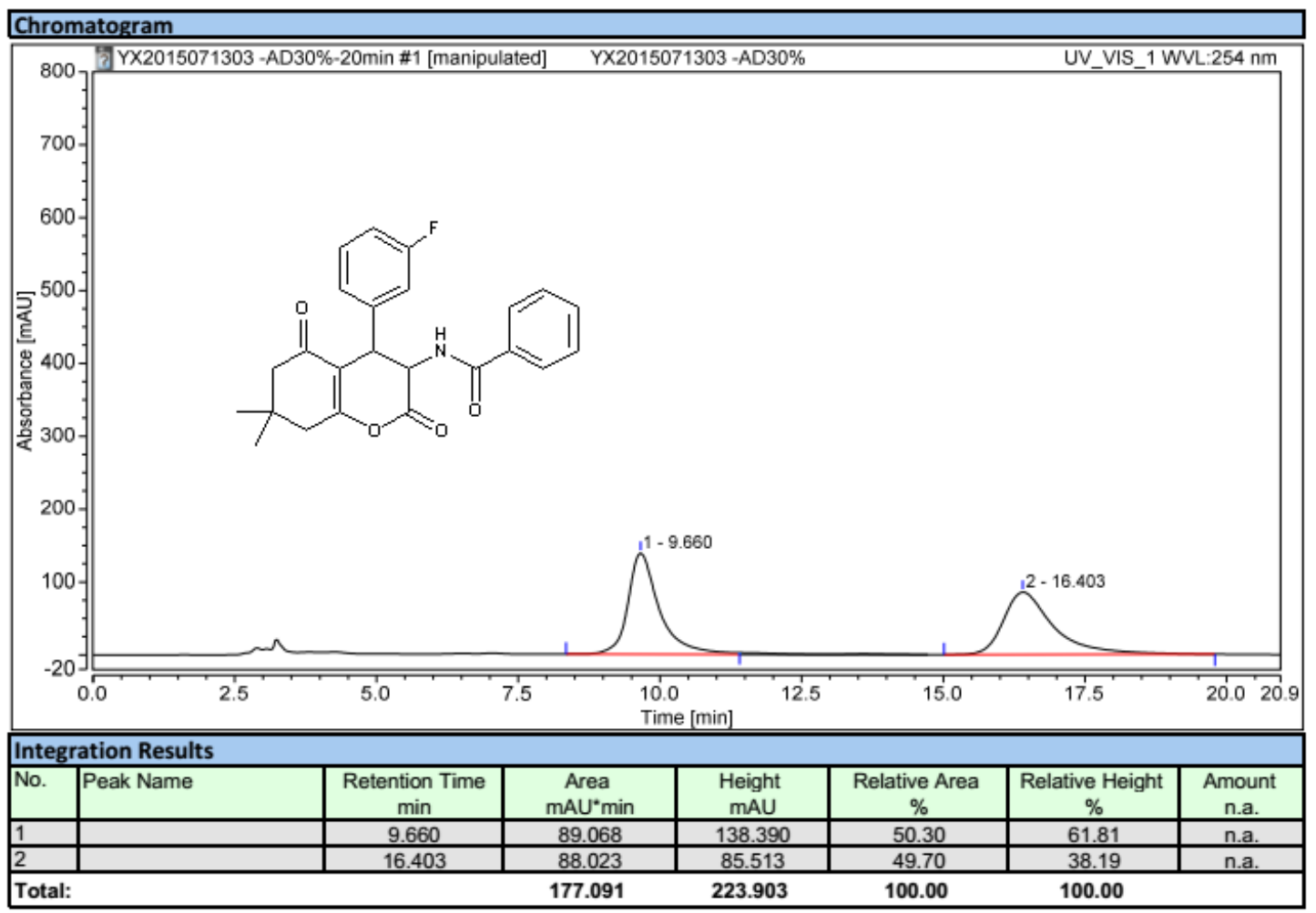

Enantioselective product:

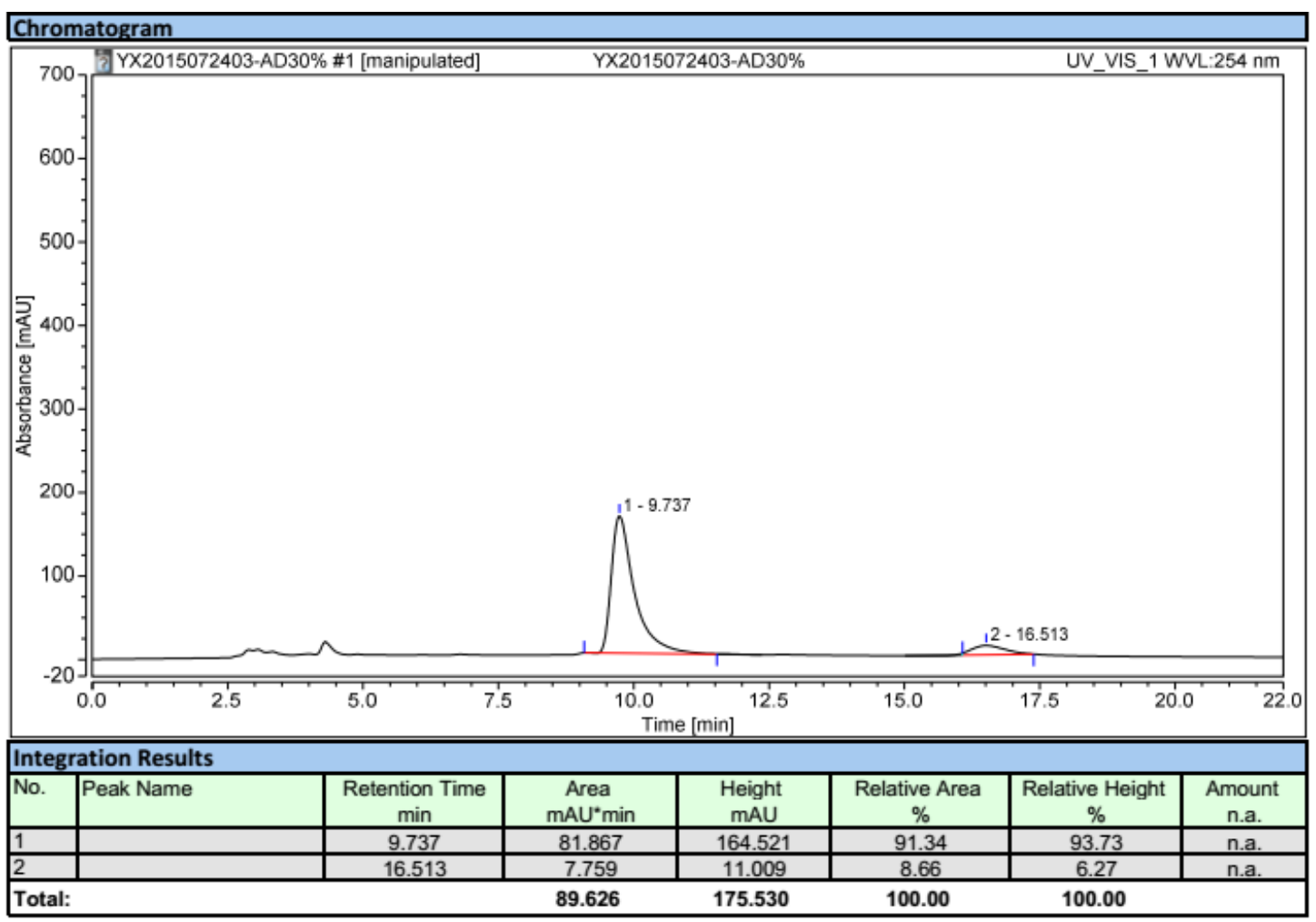




\section{3ma}

Racemic product:

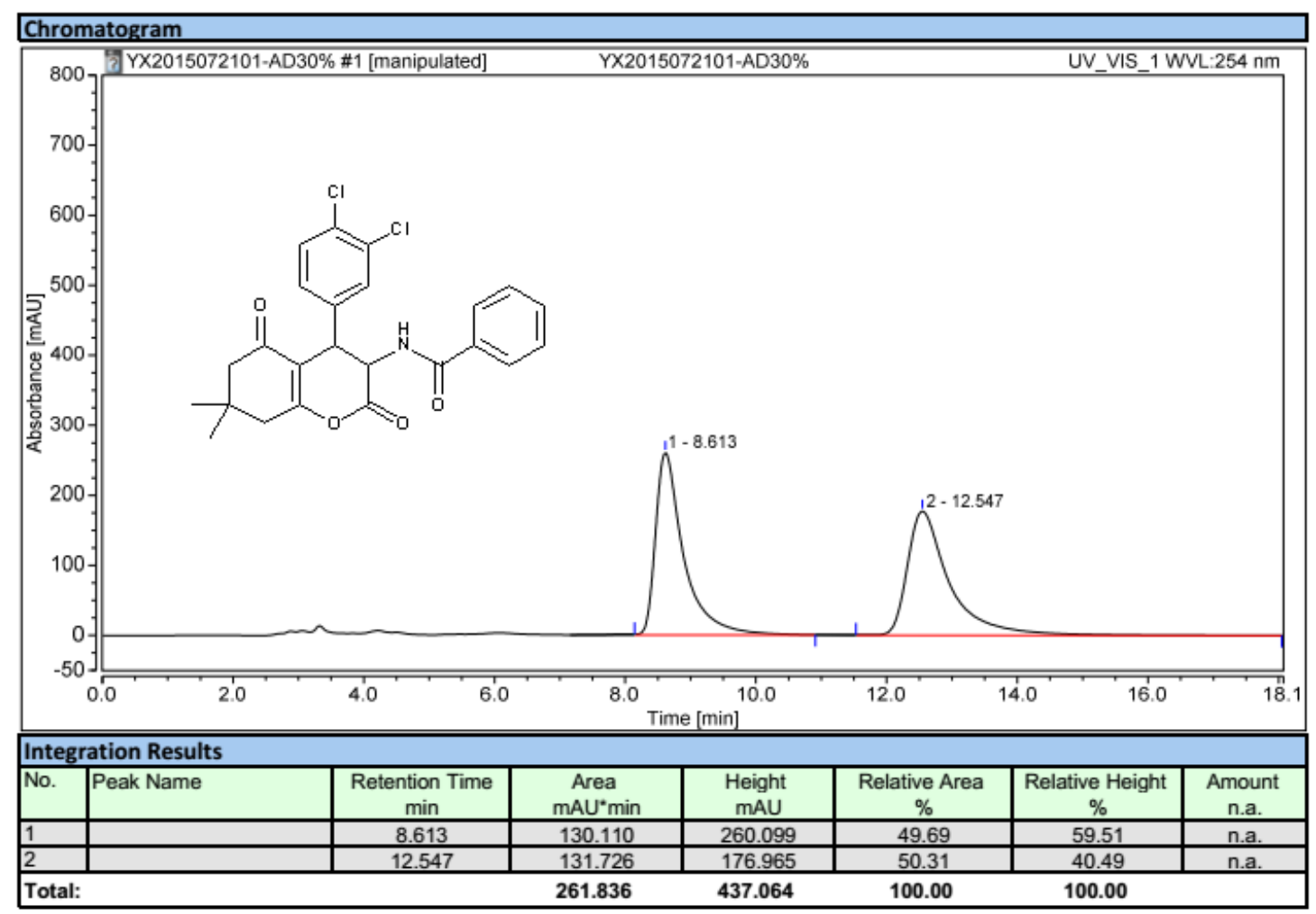

Enantioselective product:

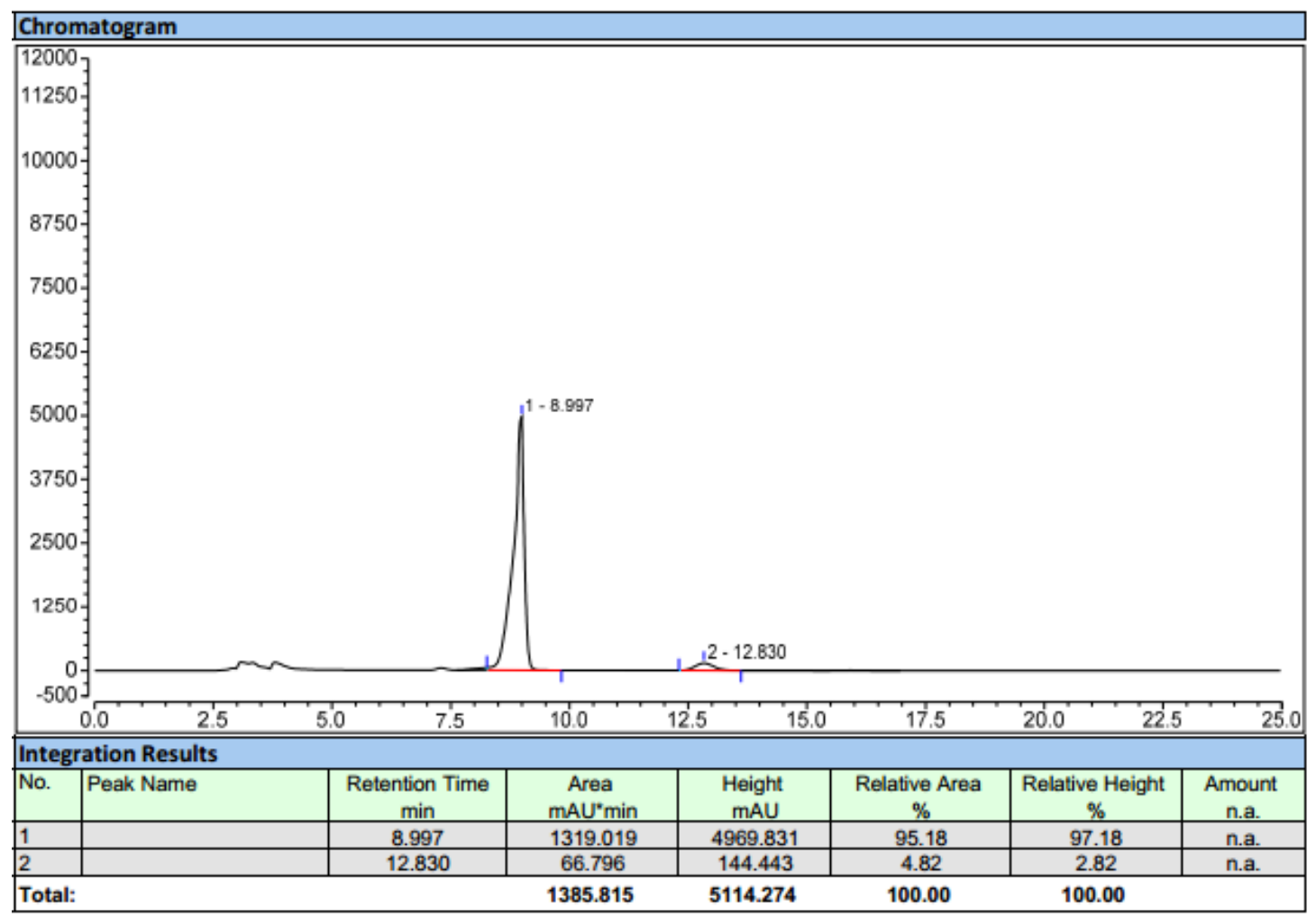




\section{3na}

Racemic product:

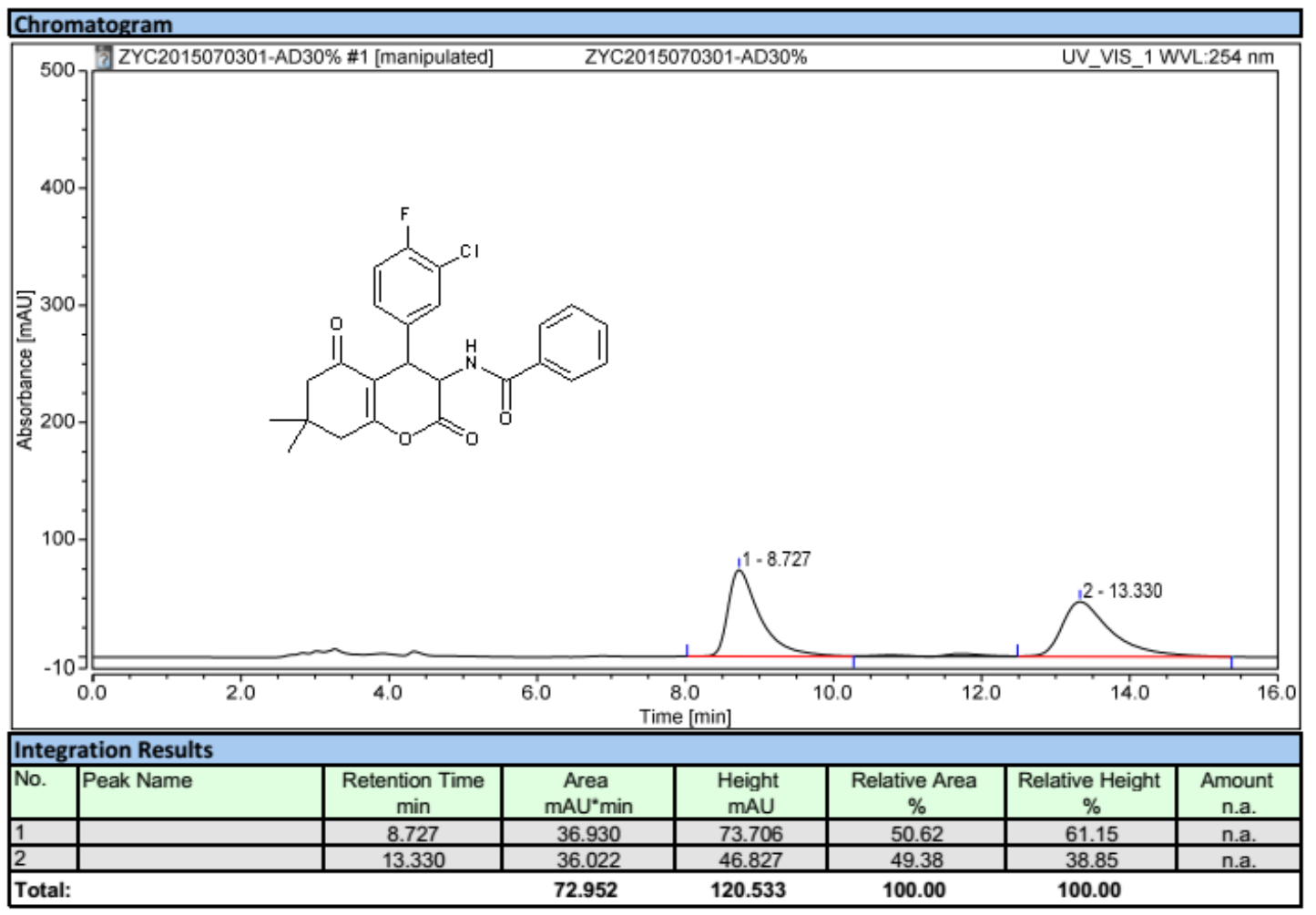

Enantioselective product:

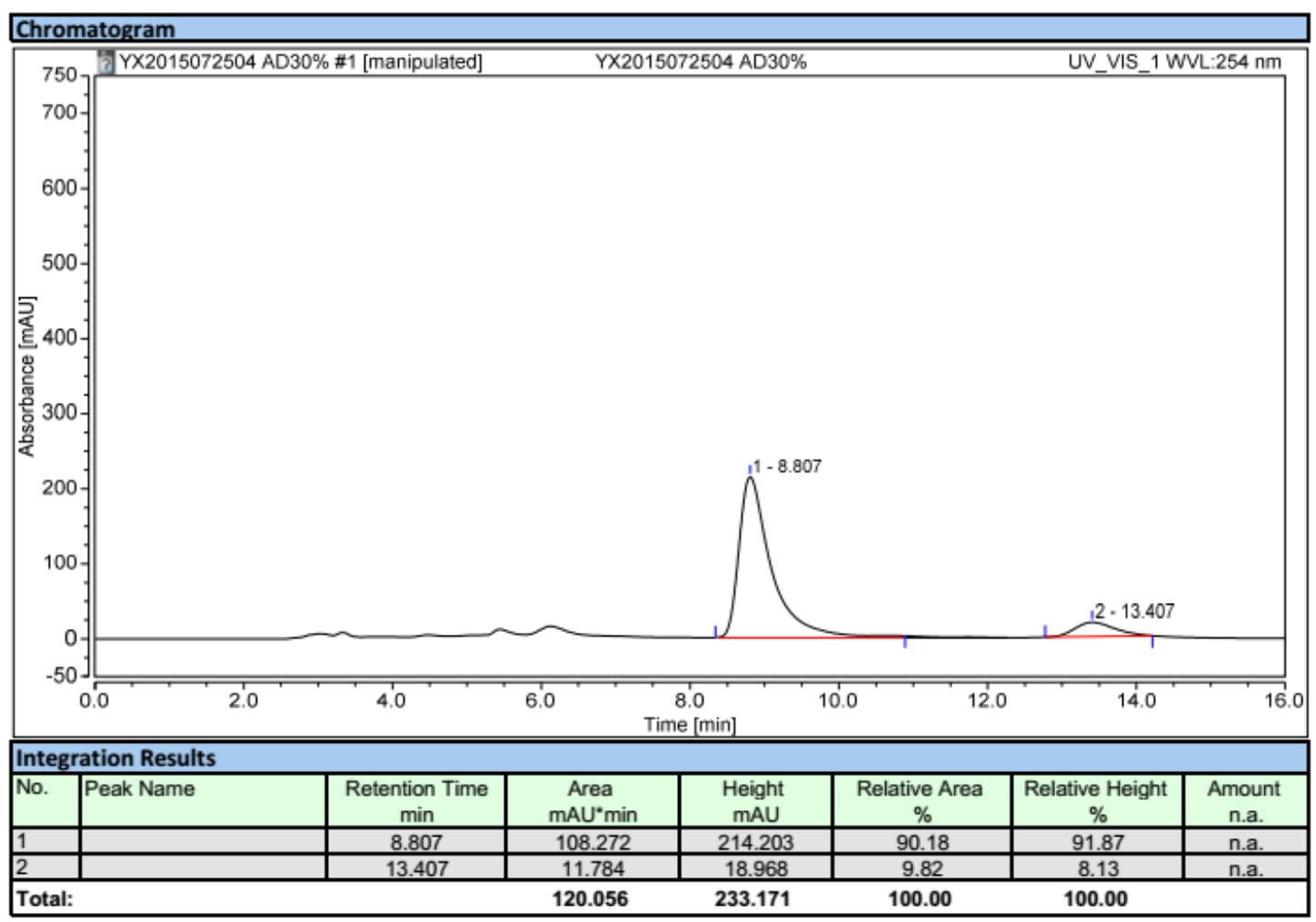


Enantioselective product after recrystallization:

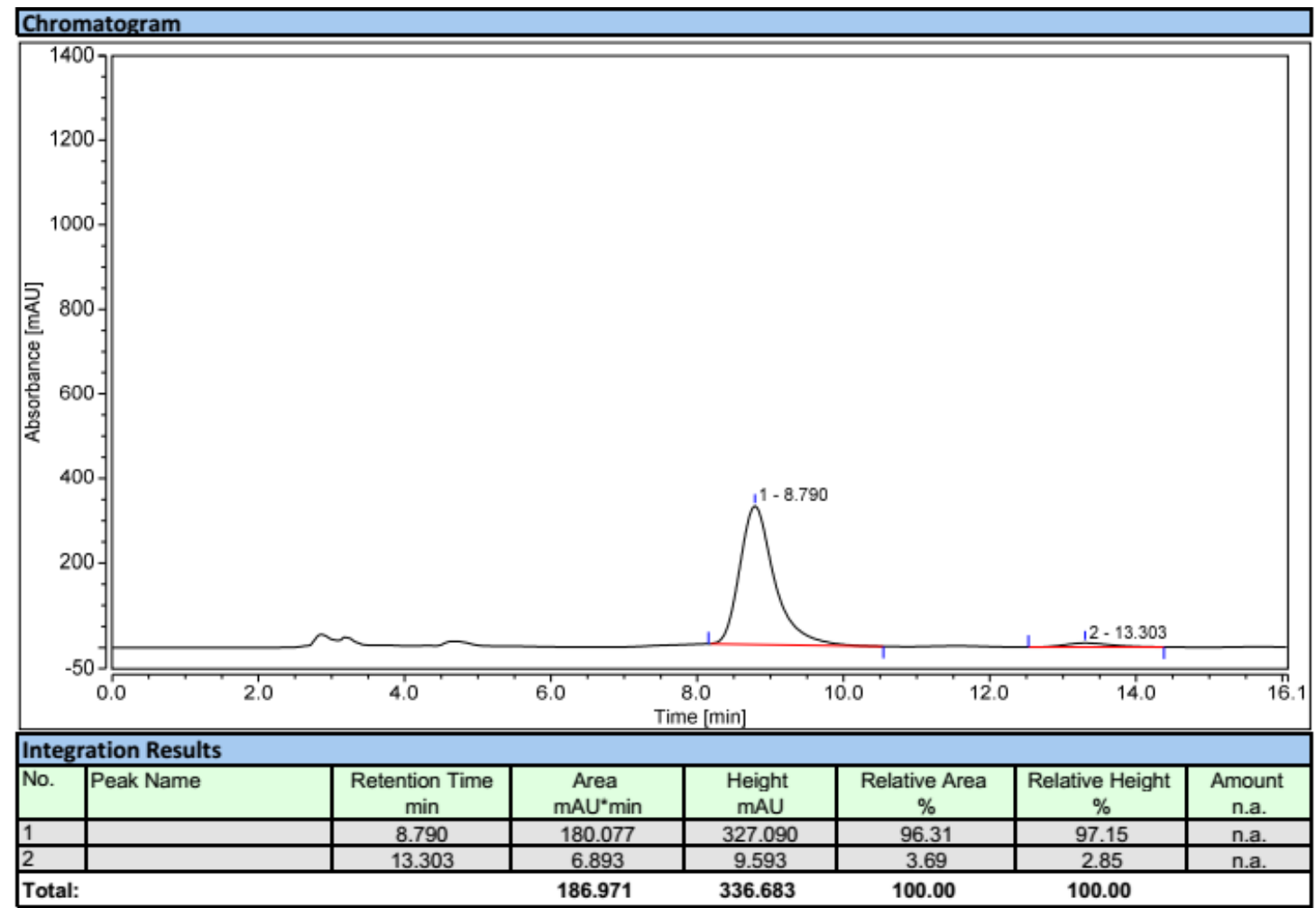




\section{3oa}

Racemic product:

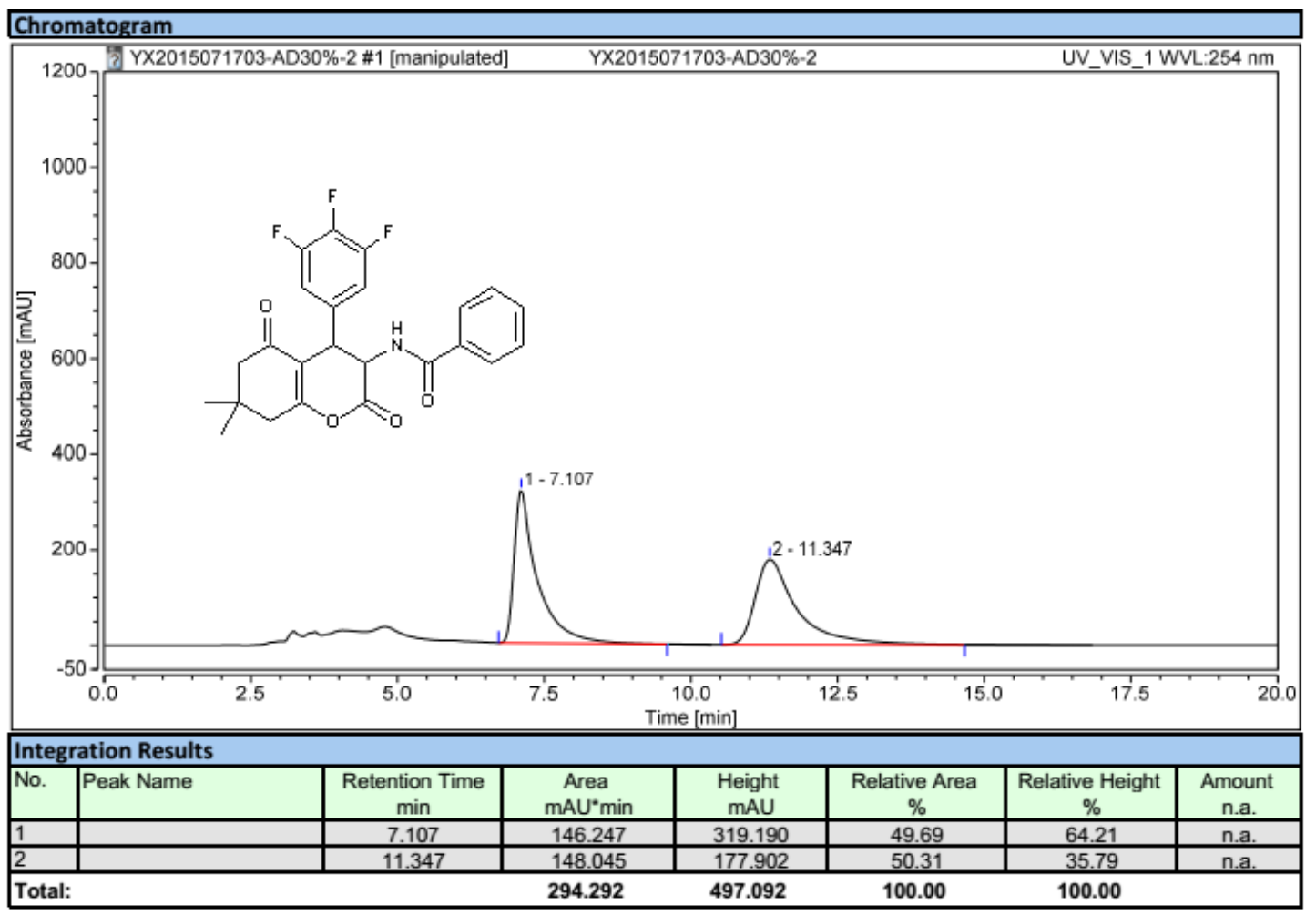

Enantioselective product:

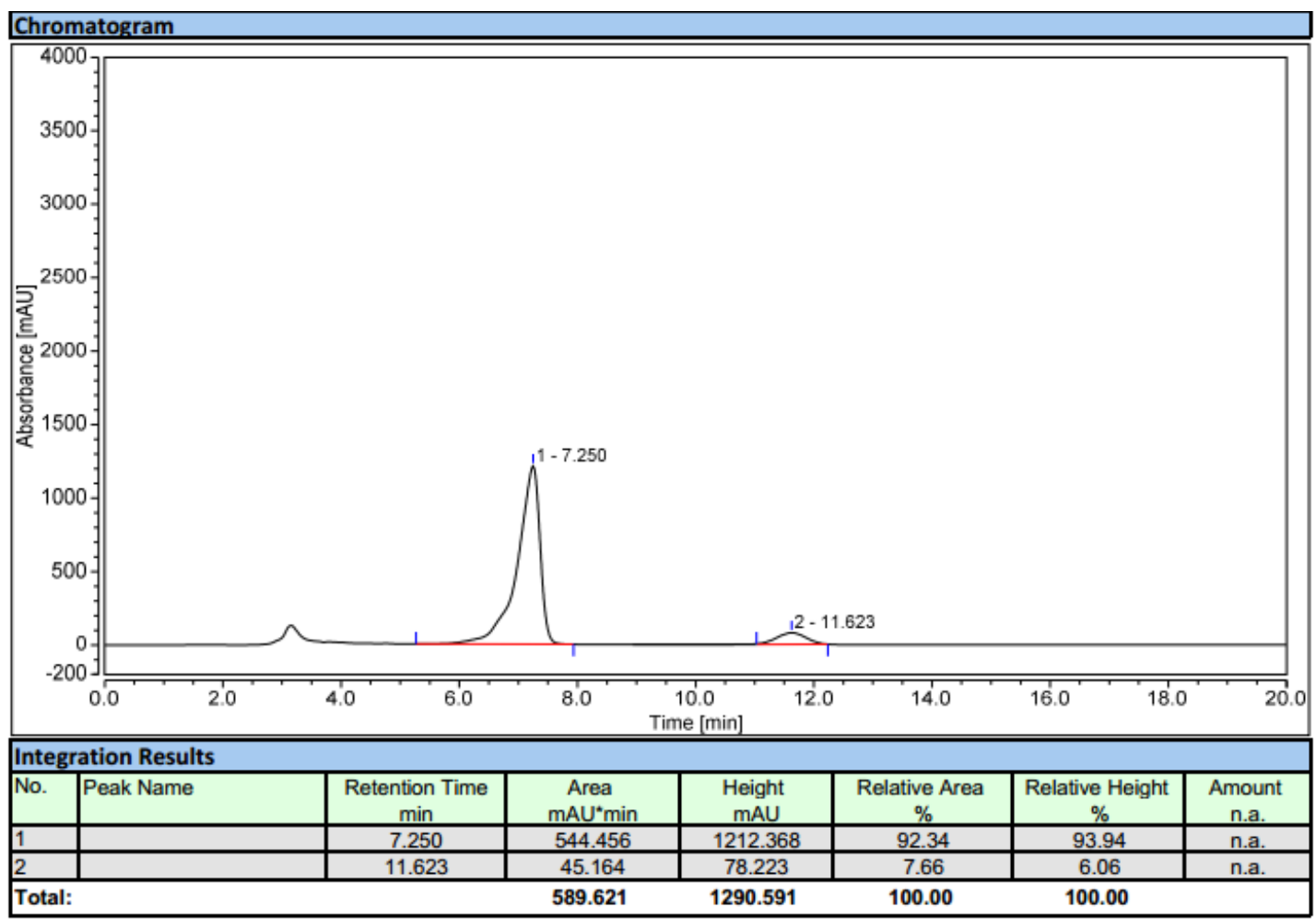




\section{3pa}

Racemic product:

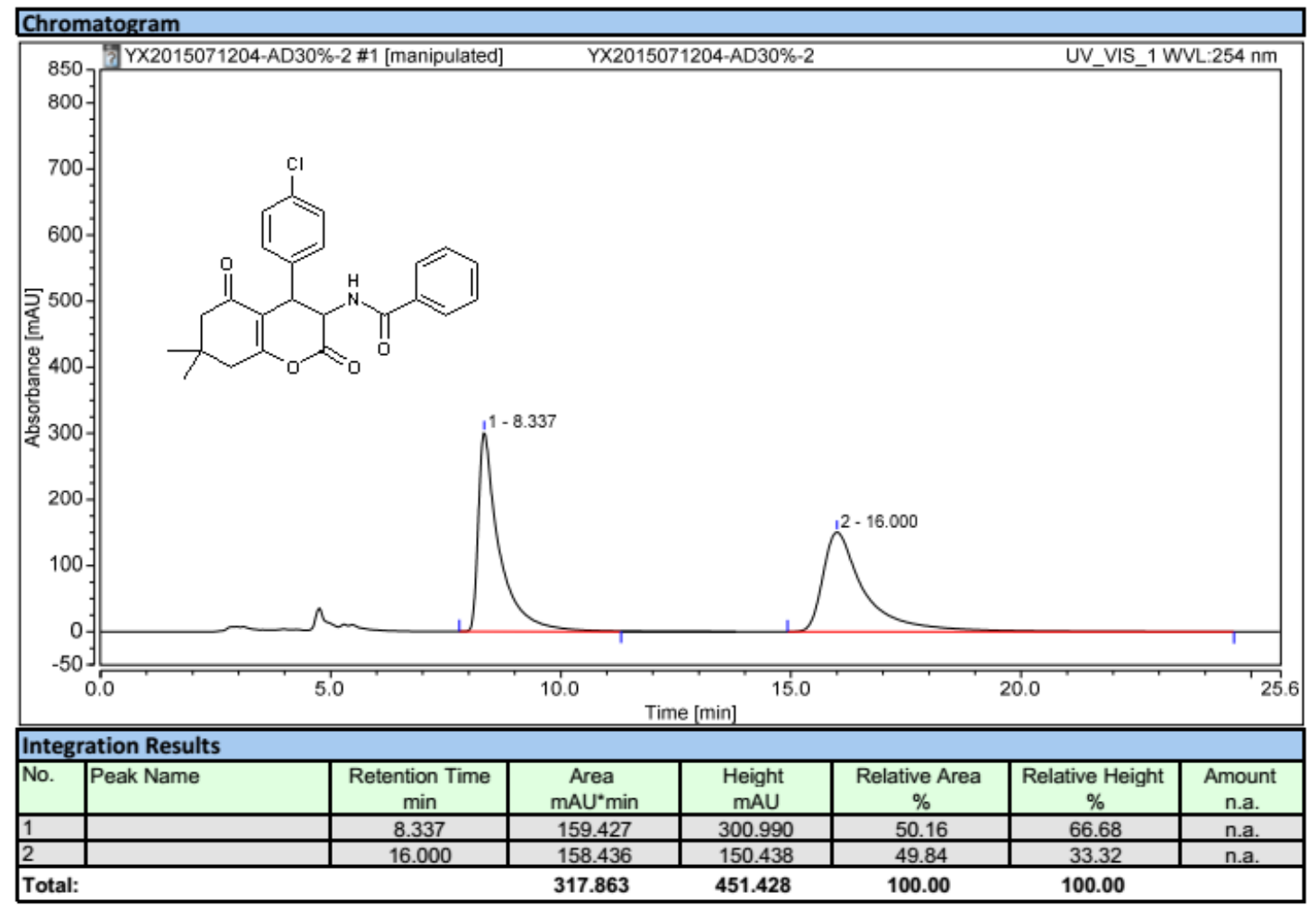

Enantioselective product:

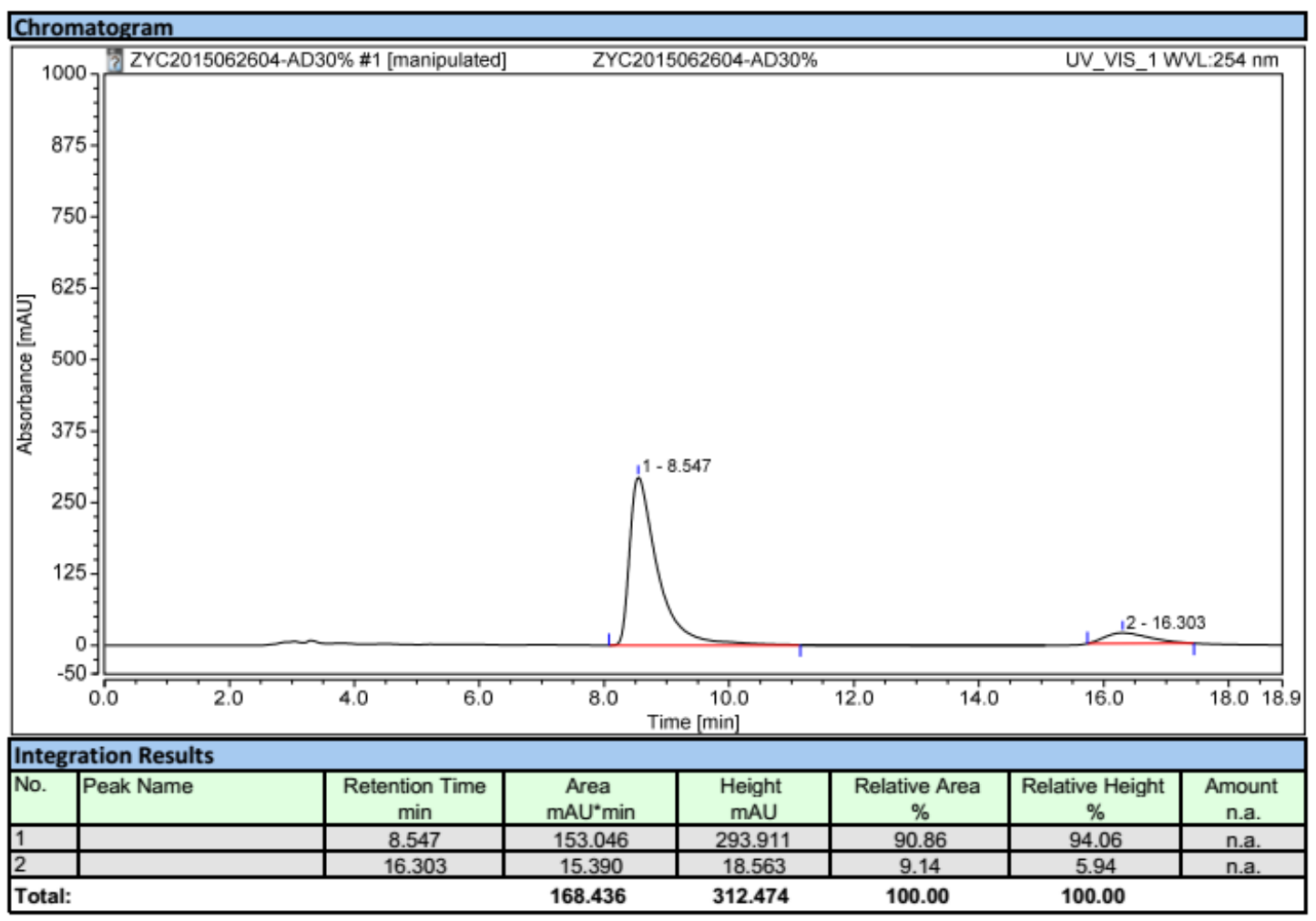


Enantioselective product after recrystallization:

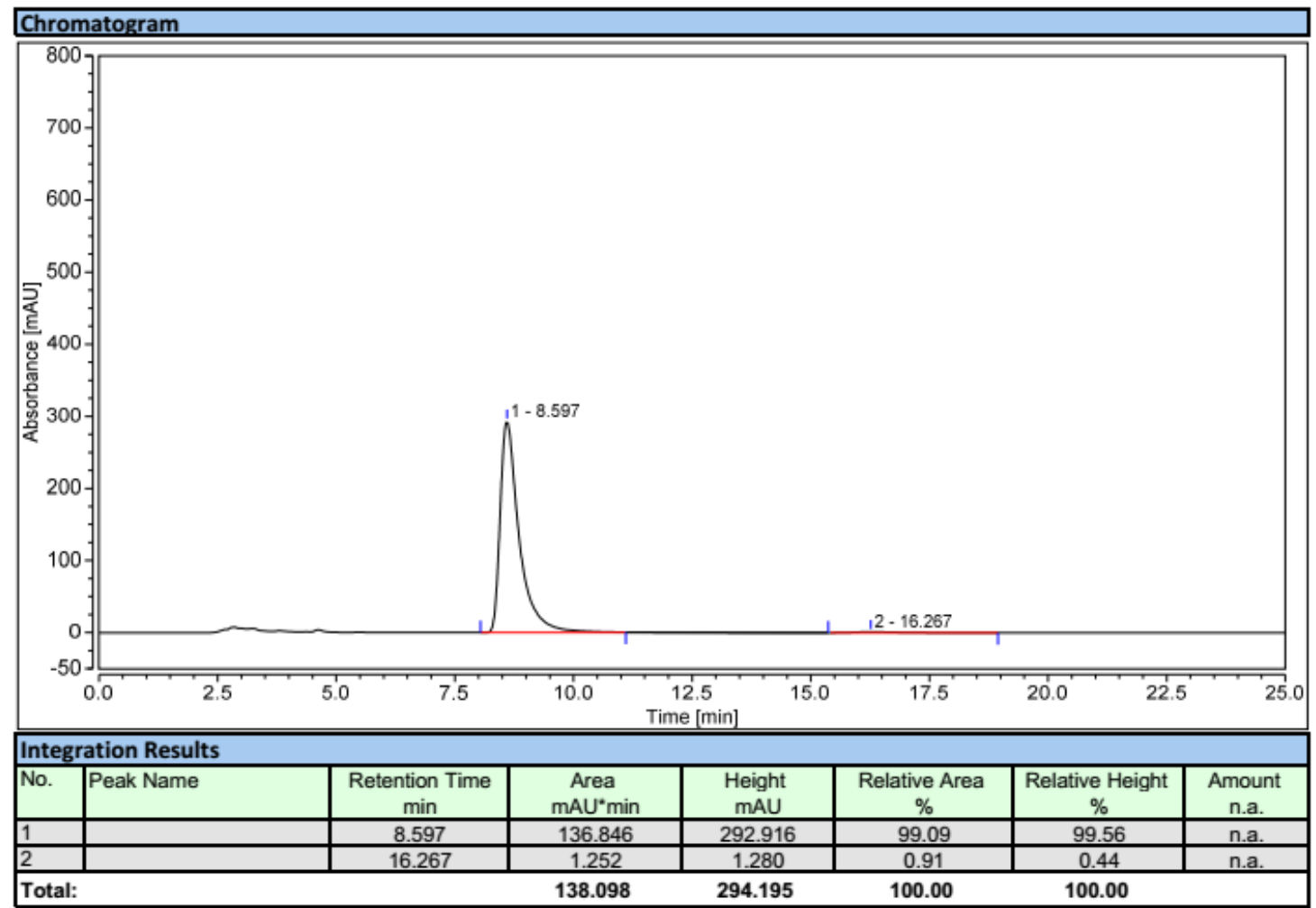




\section{$3 q a$}

Racemic product:

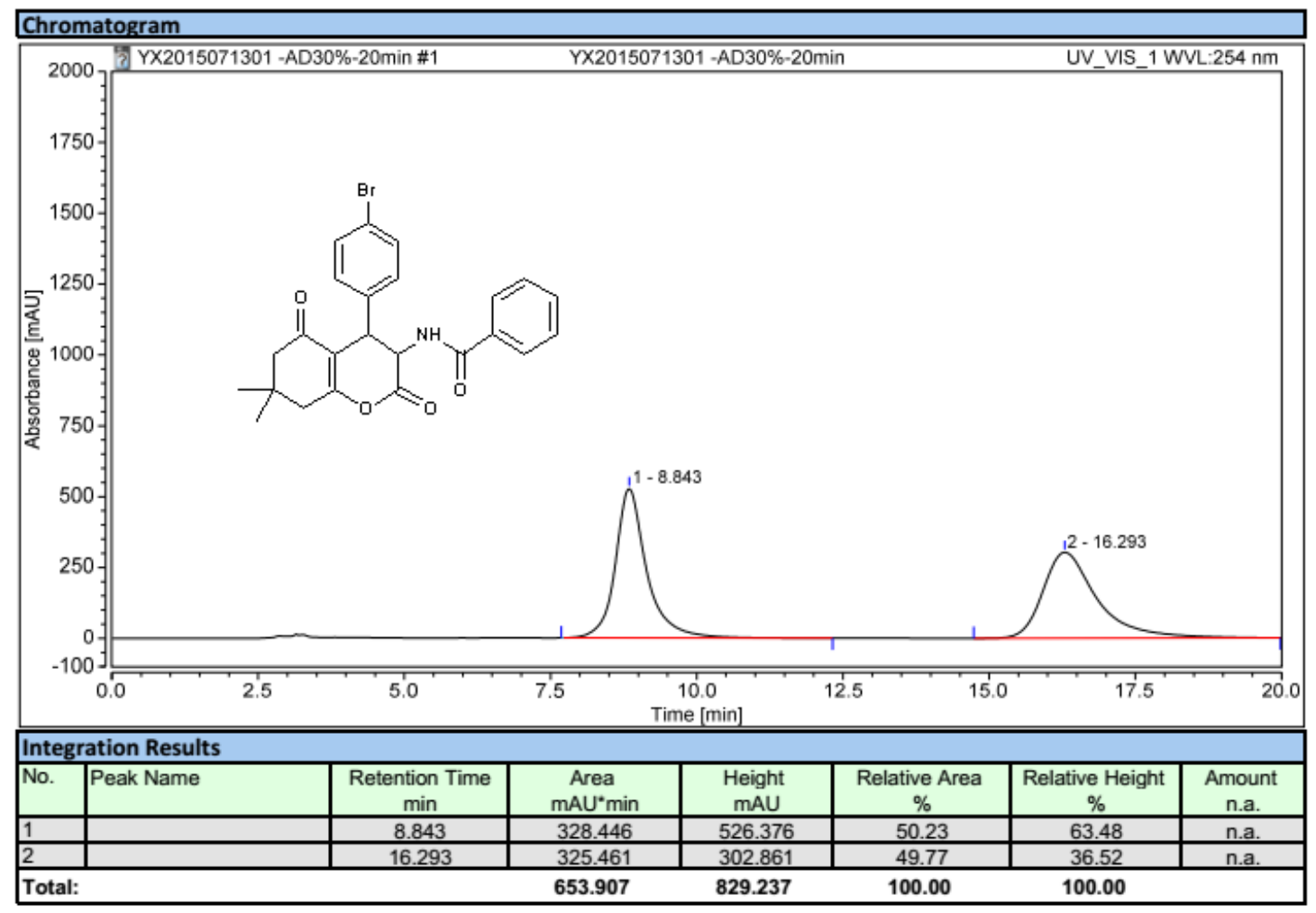

Enantioselective product:

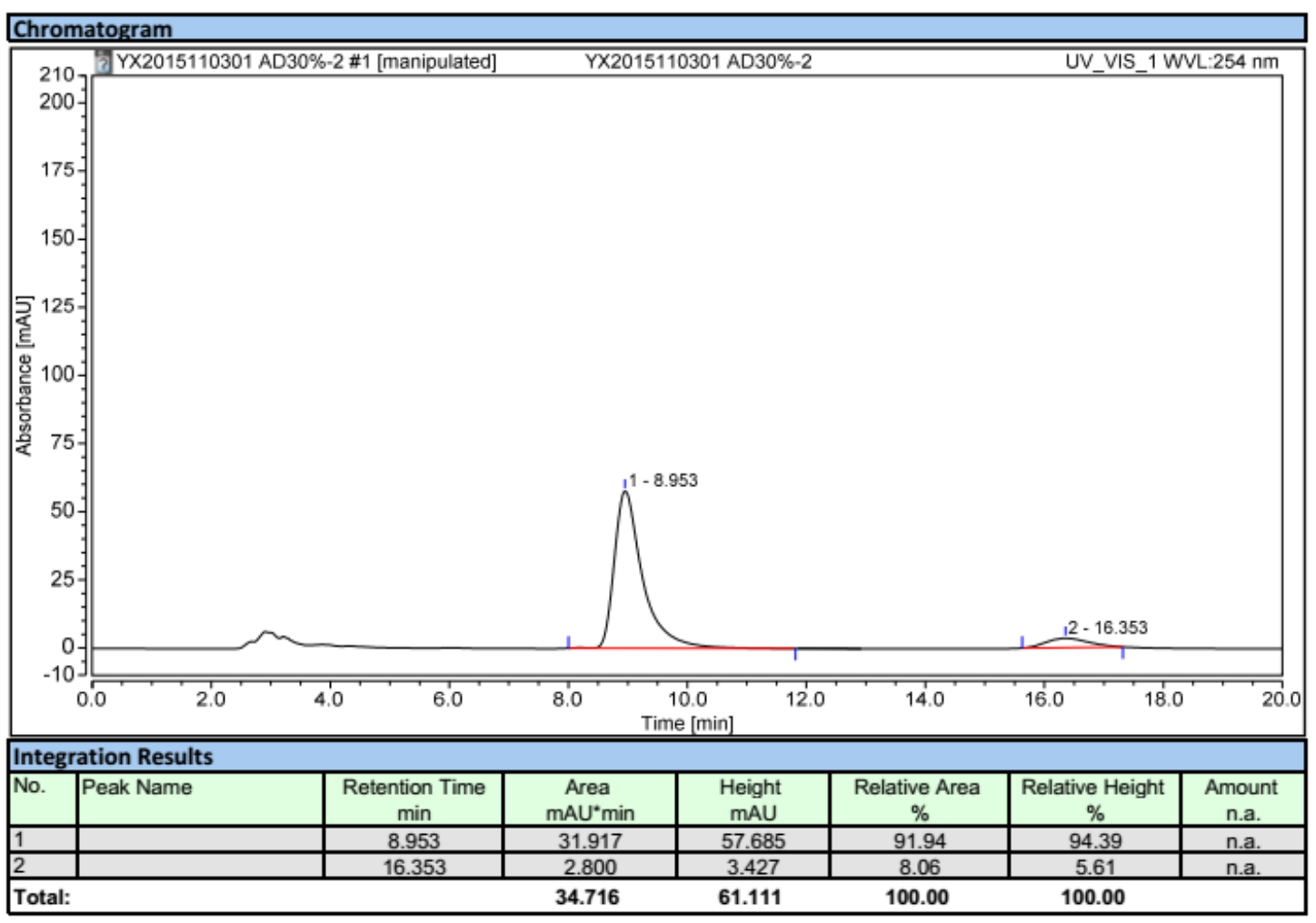


Enantioselective product after recrystallization:

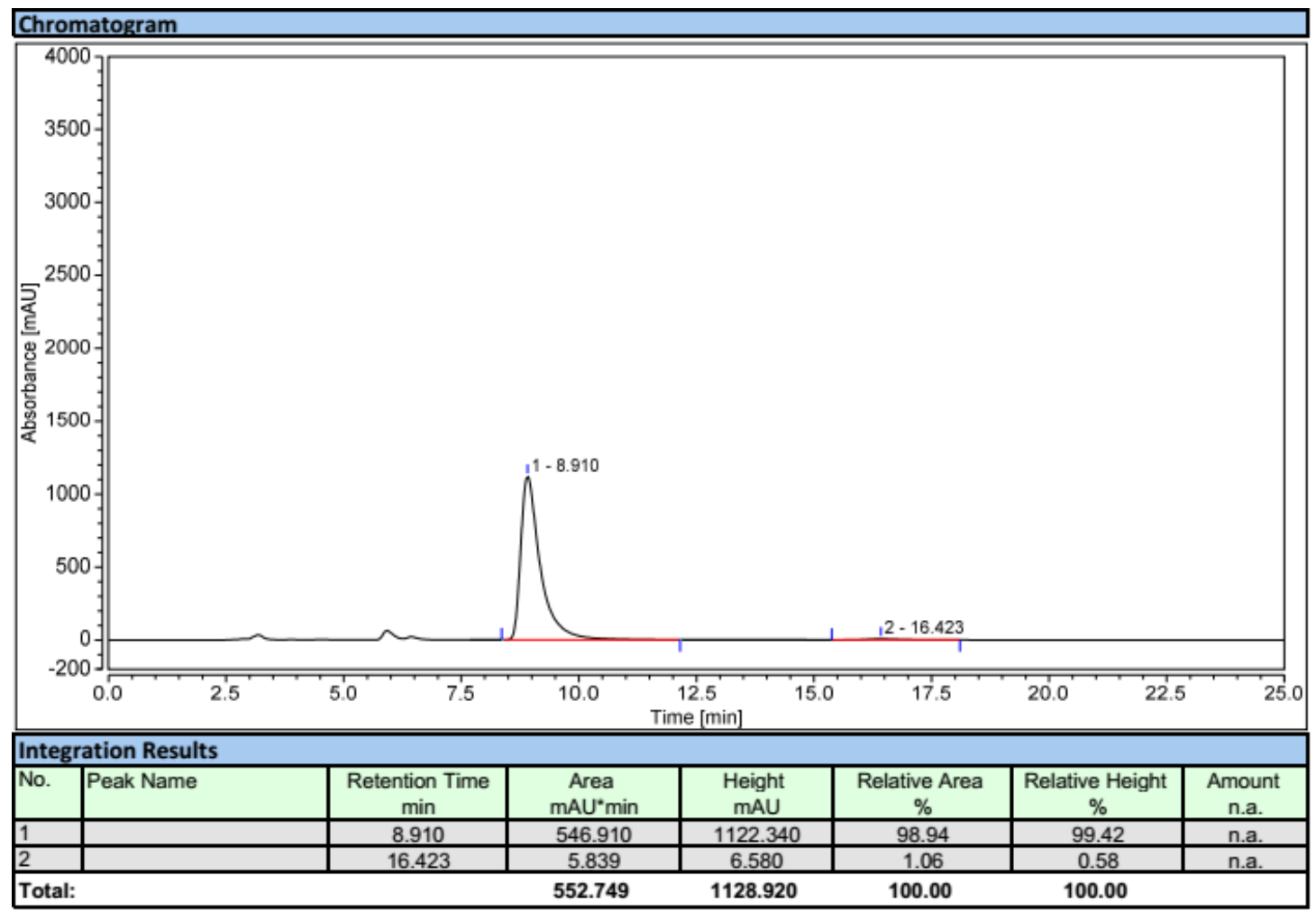




\section{3ra}

Racemic product:

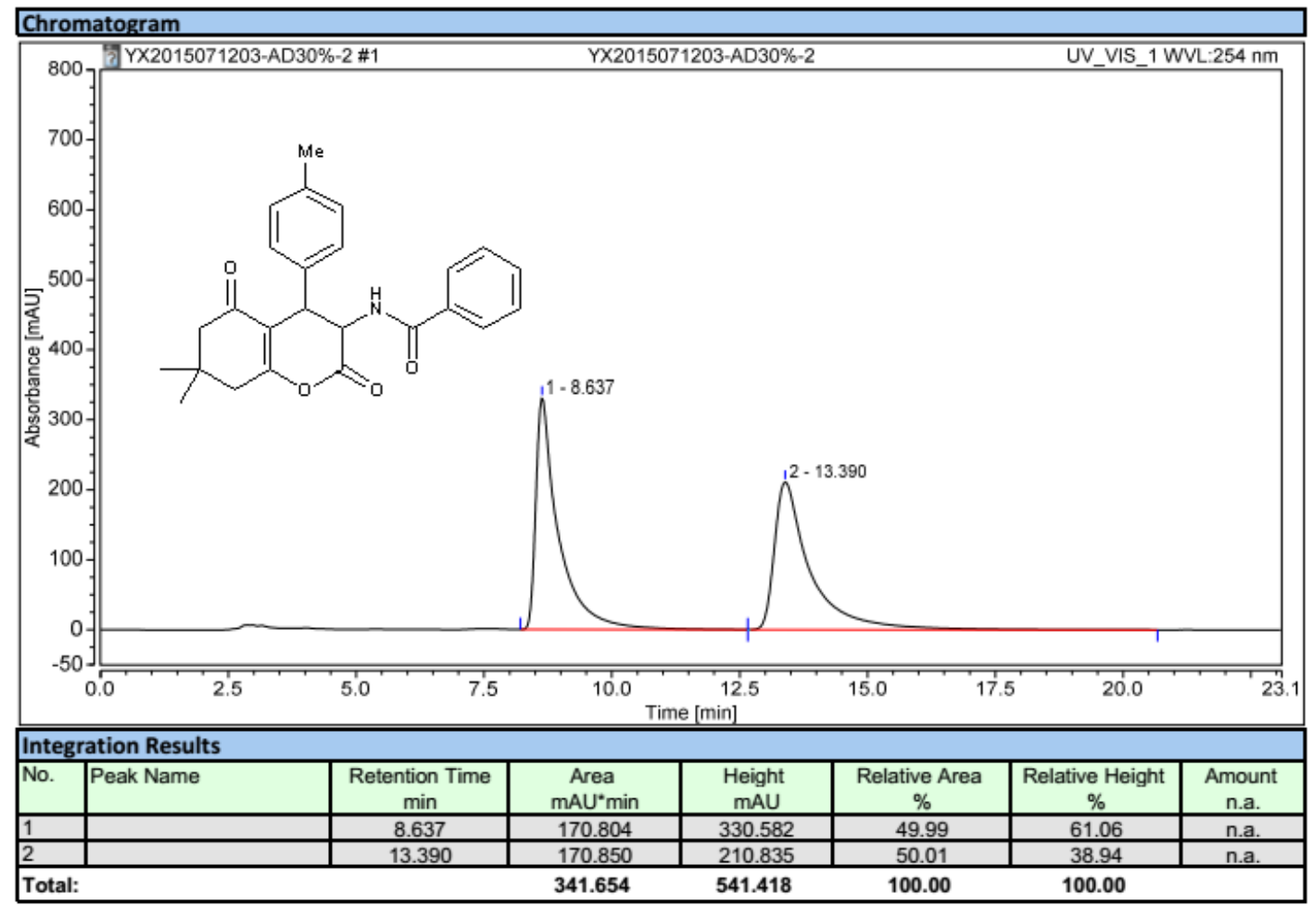

Enantioselective product:

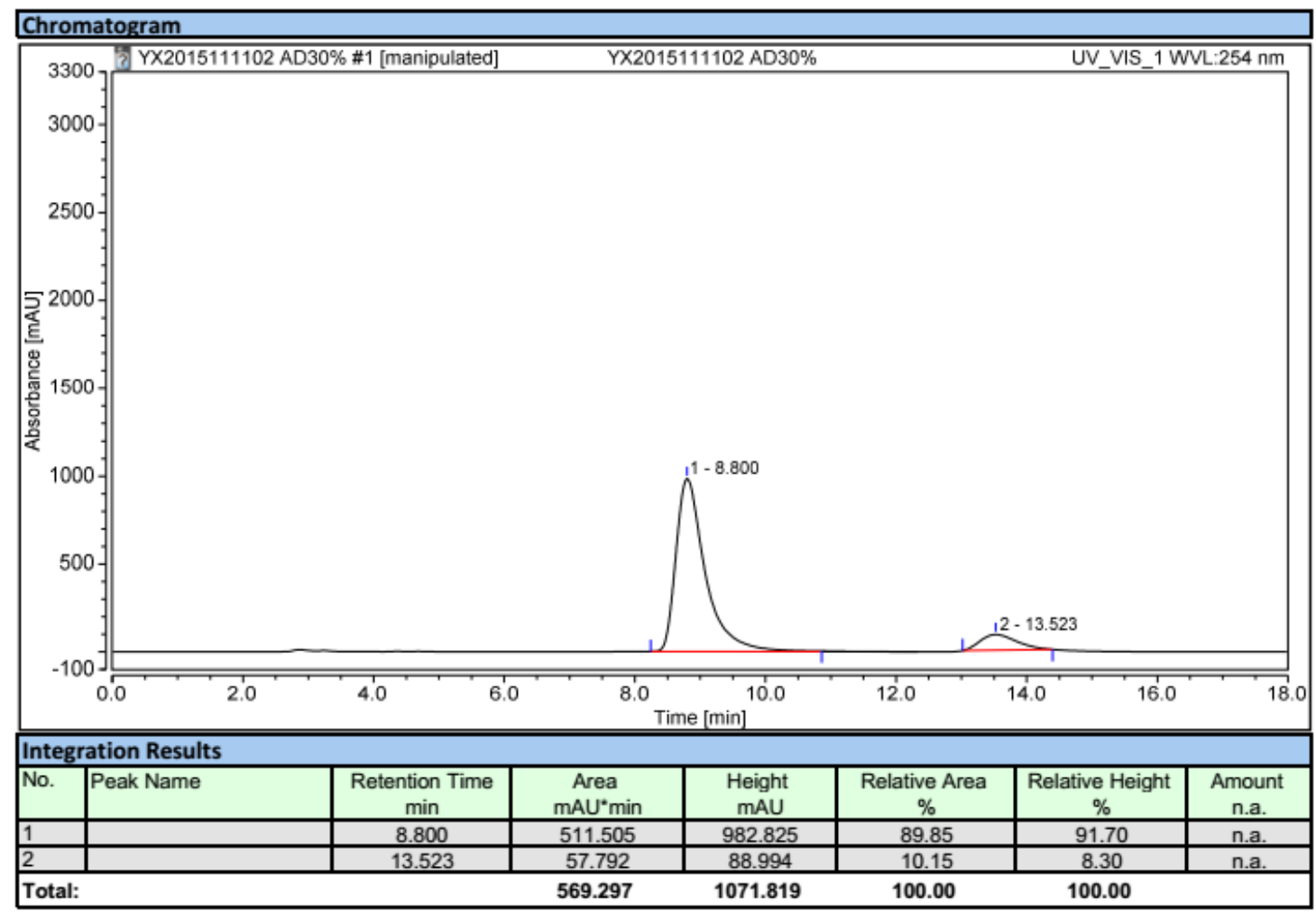


Enantioselective product after recrystallization:

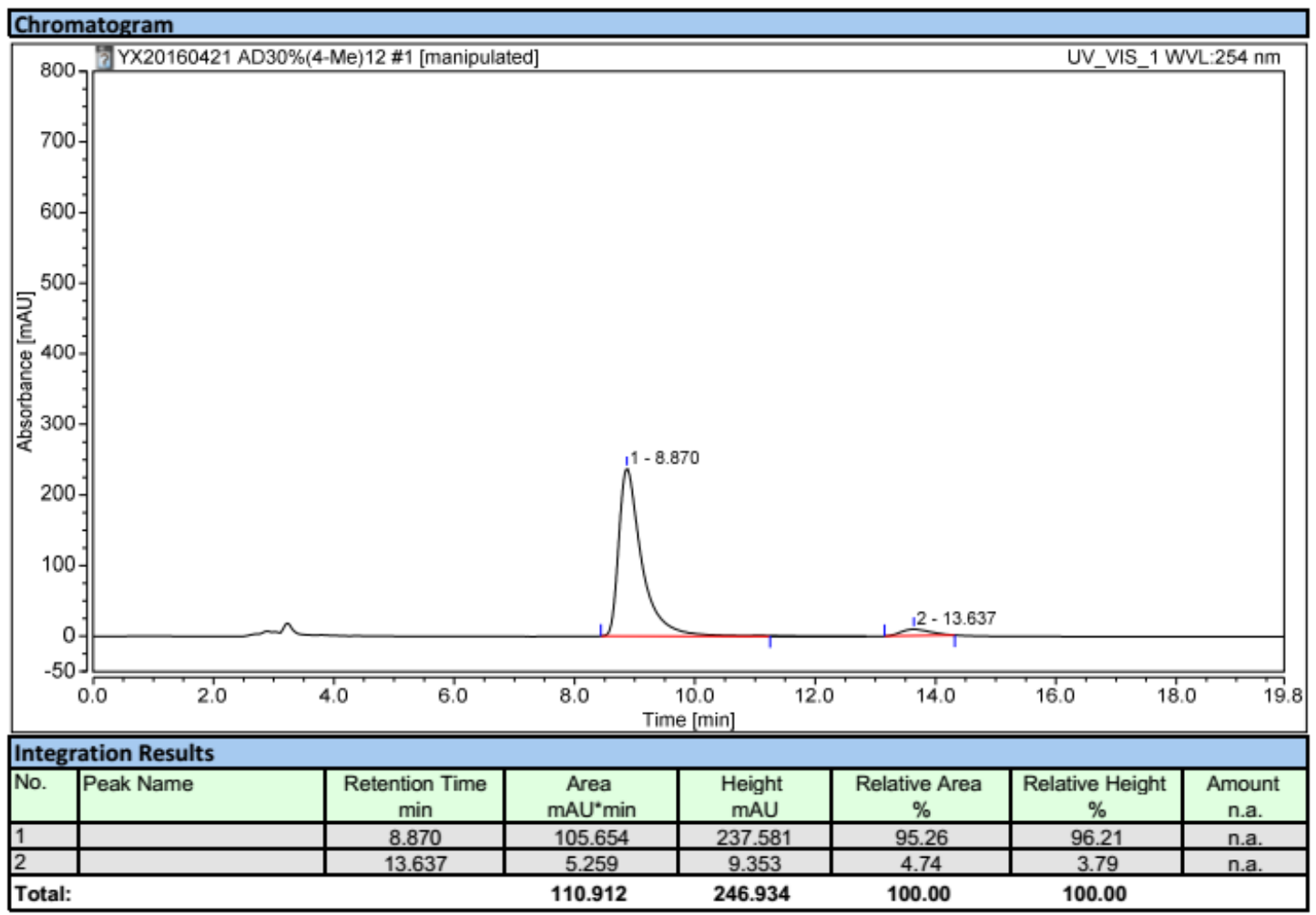




\section{3sa}

Racemic product:

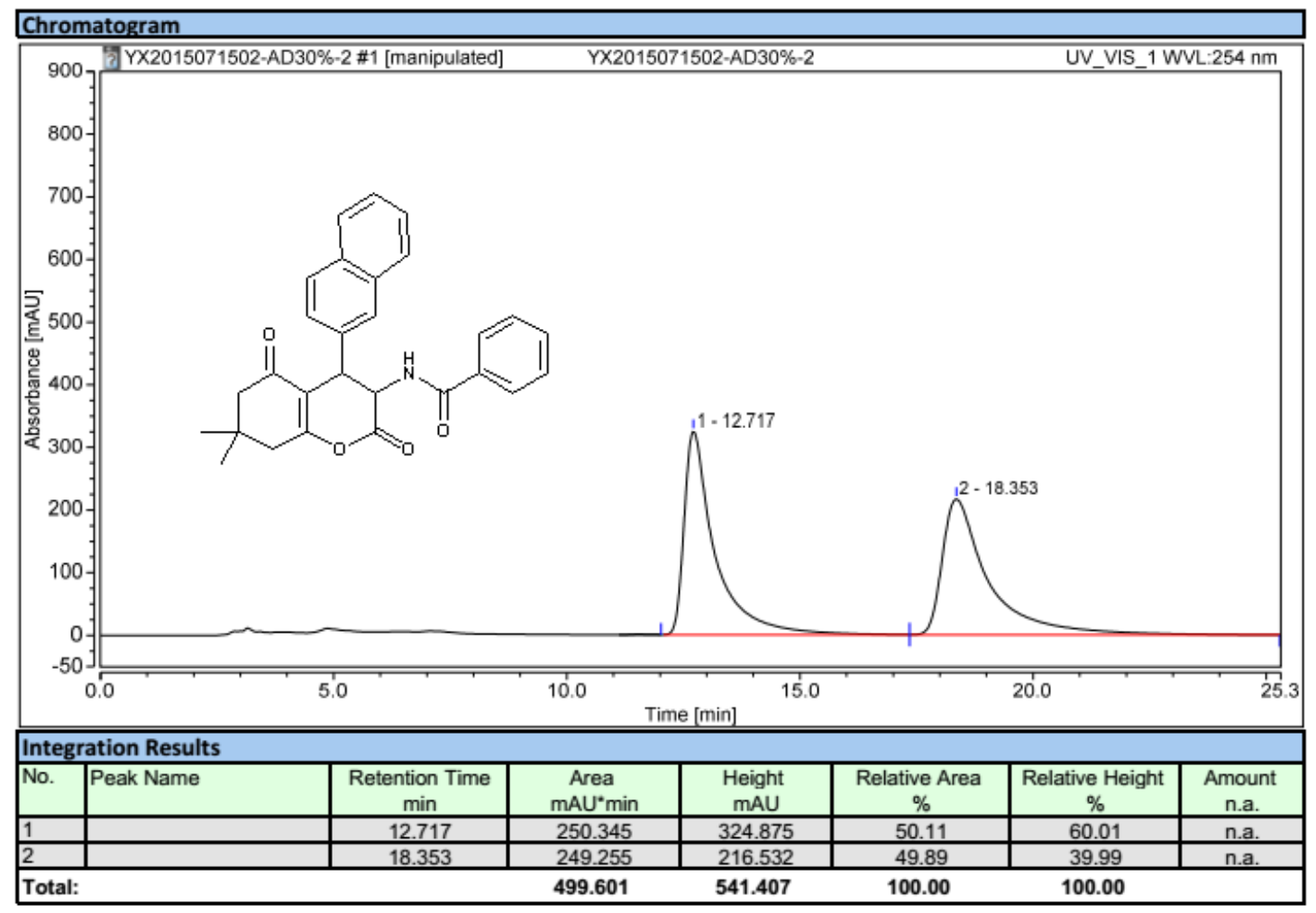

Enantioselective product:

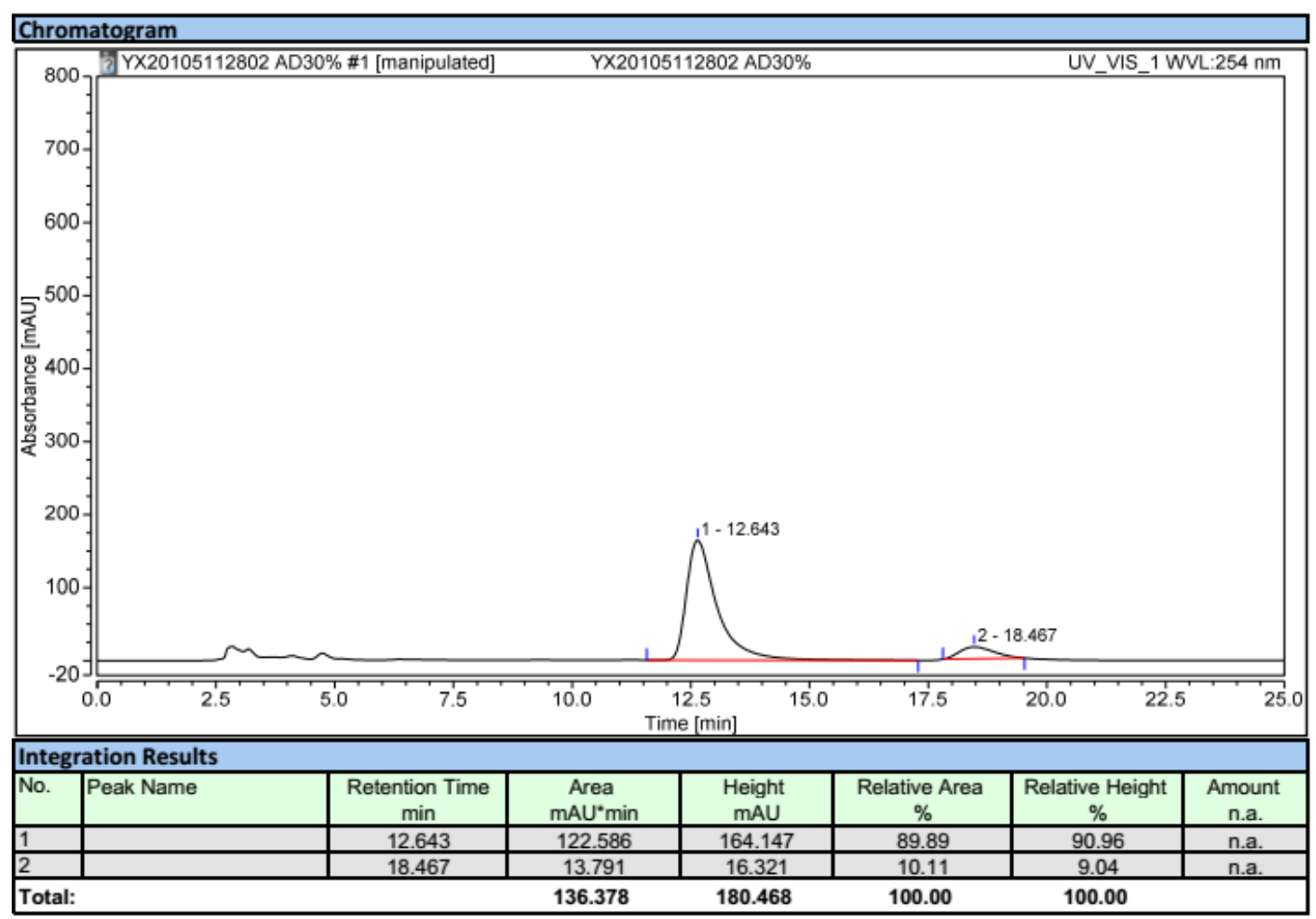




\section{3ta}

Racemic product:

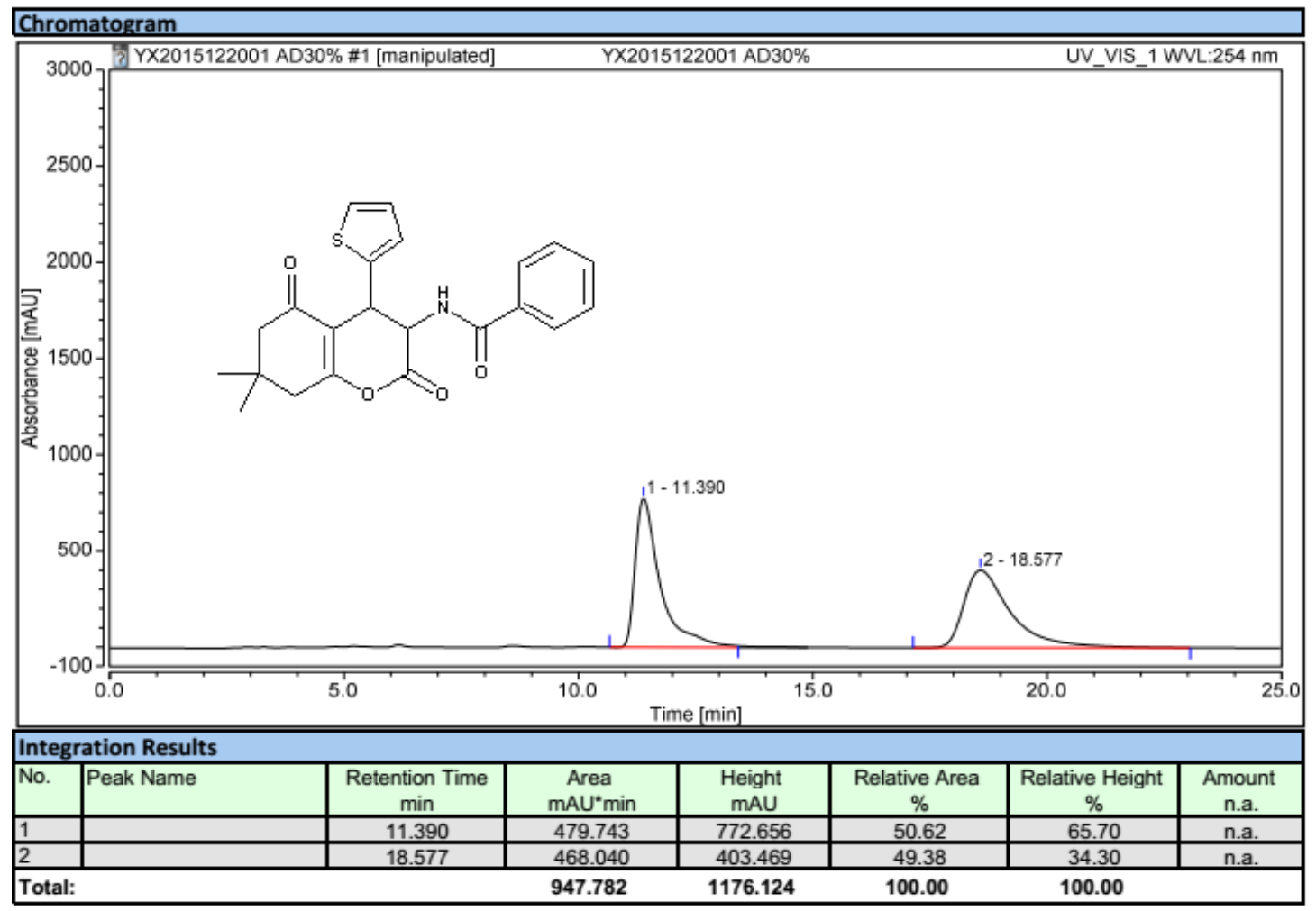

Enantioselective product:

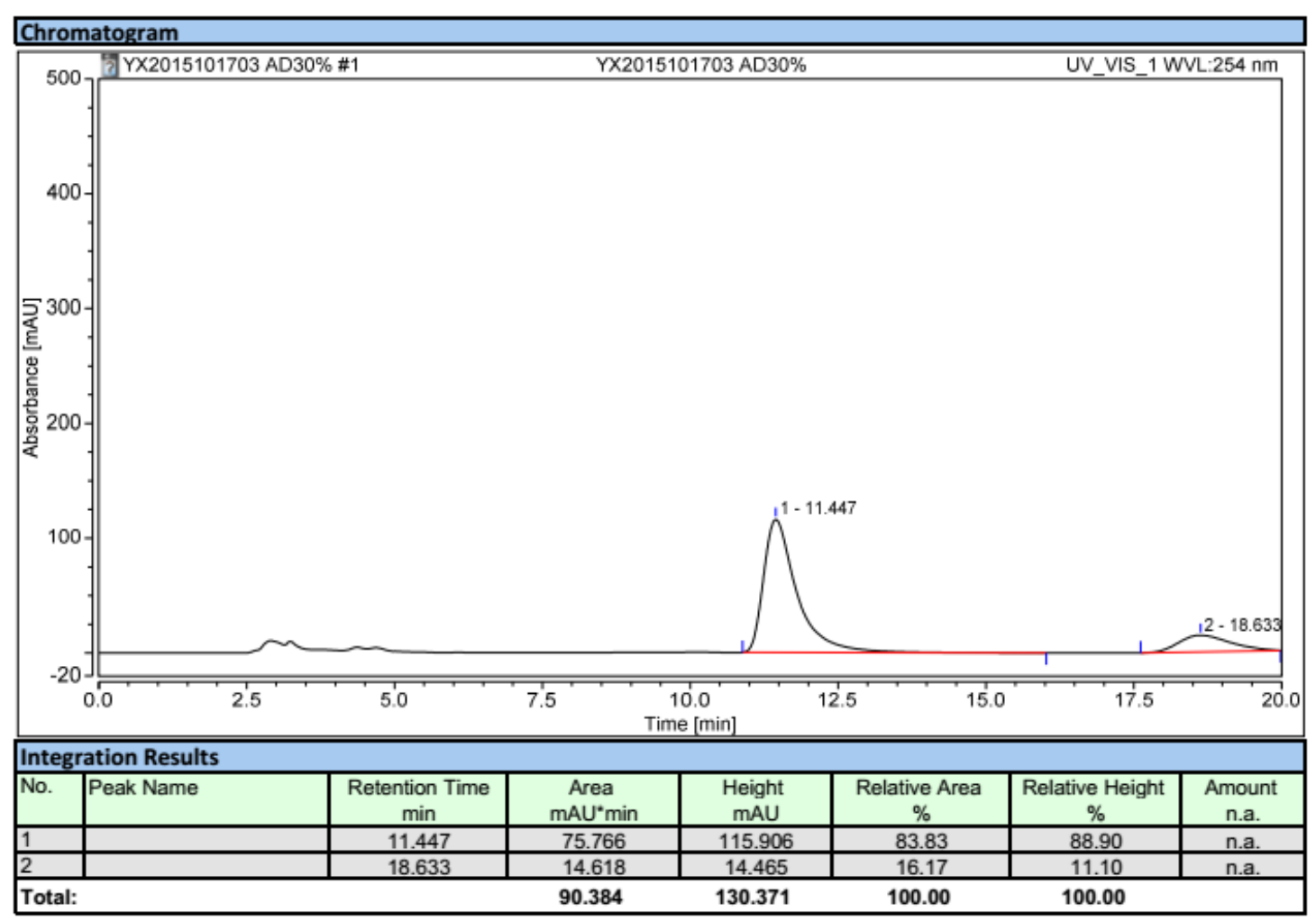




\section{3ab}

Racemic product:

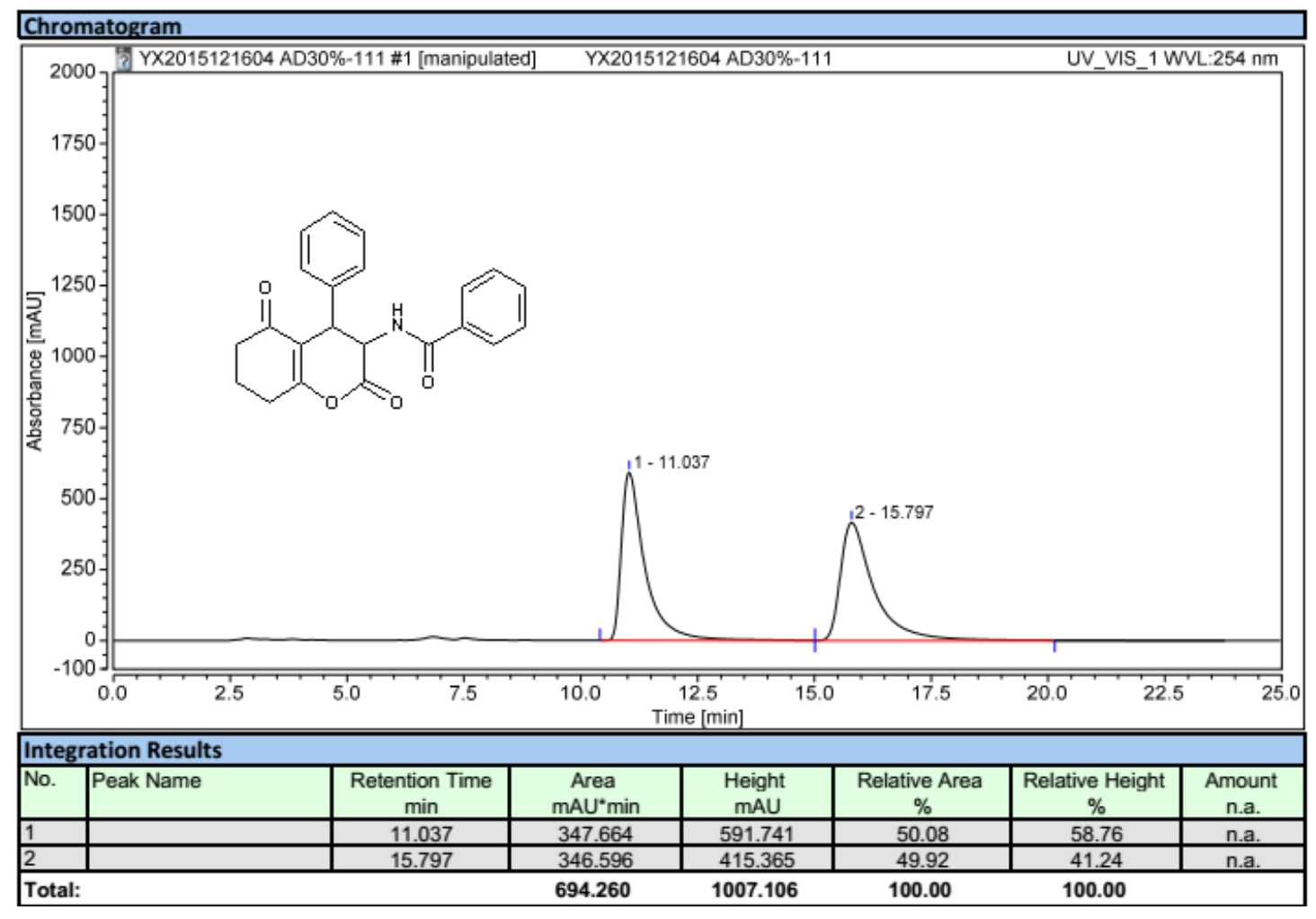

Enantioselective product:

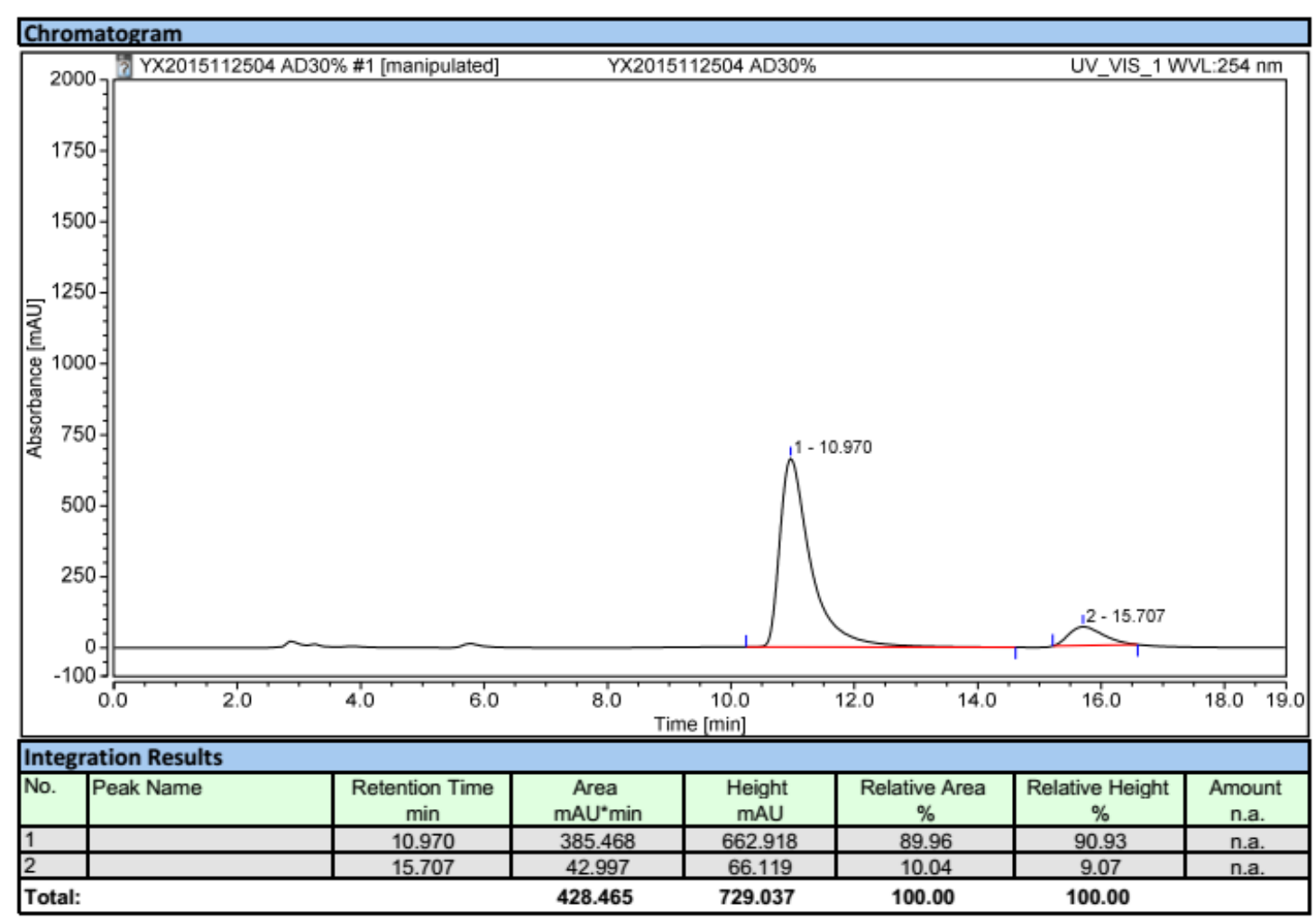


Enantioselective product after recrystallization:

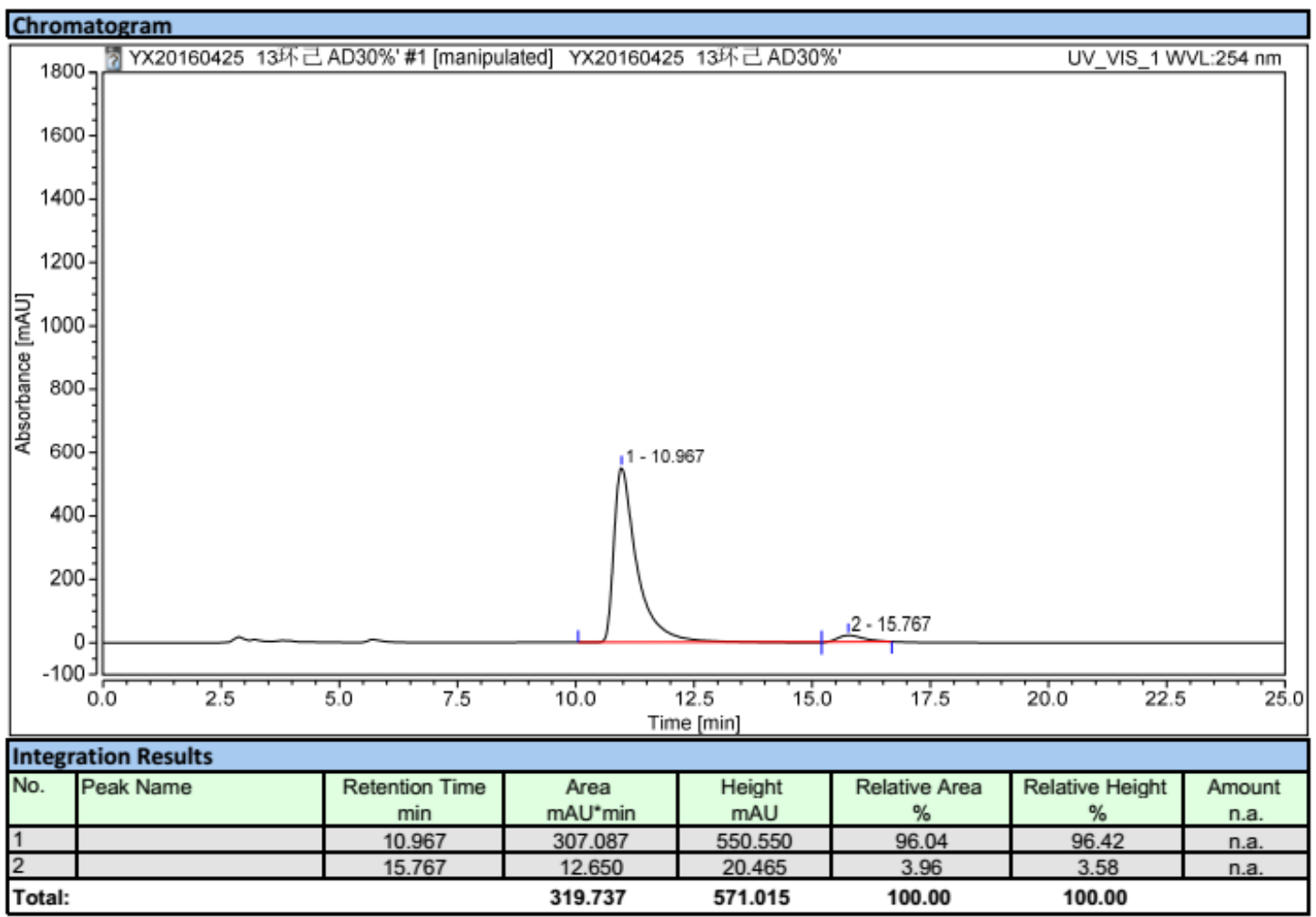




\section{3ad}

Racemic product:

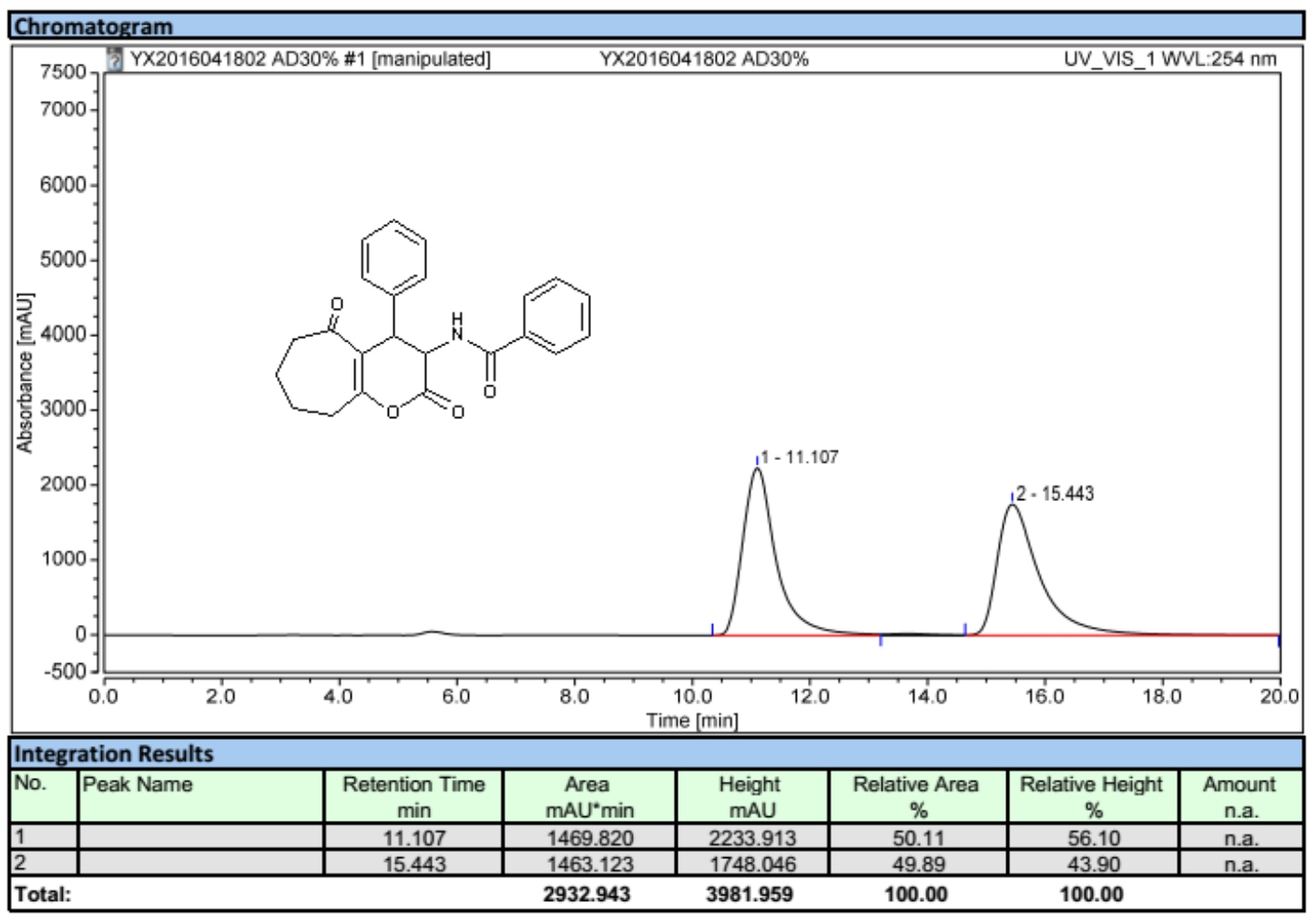

Enantioselective product:

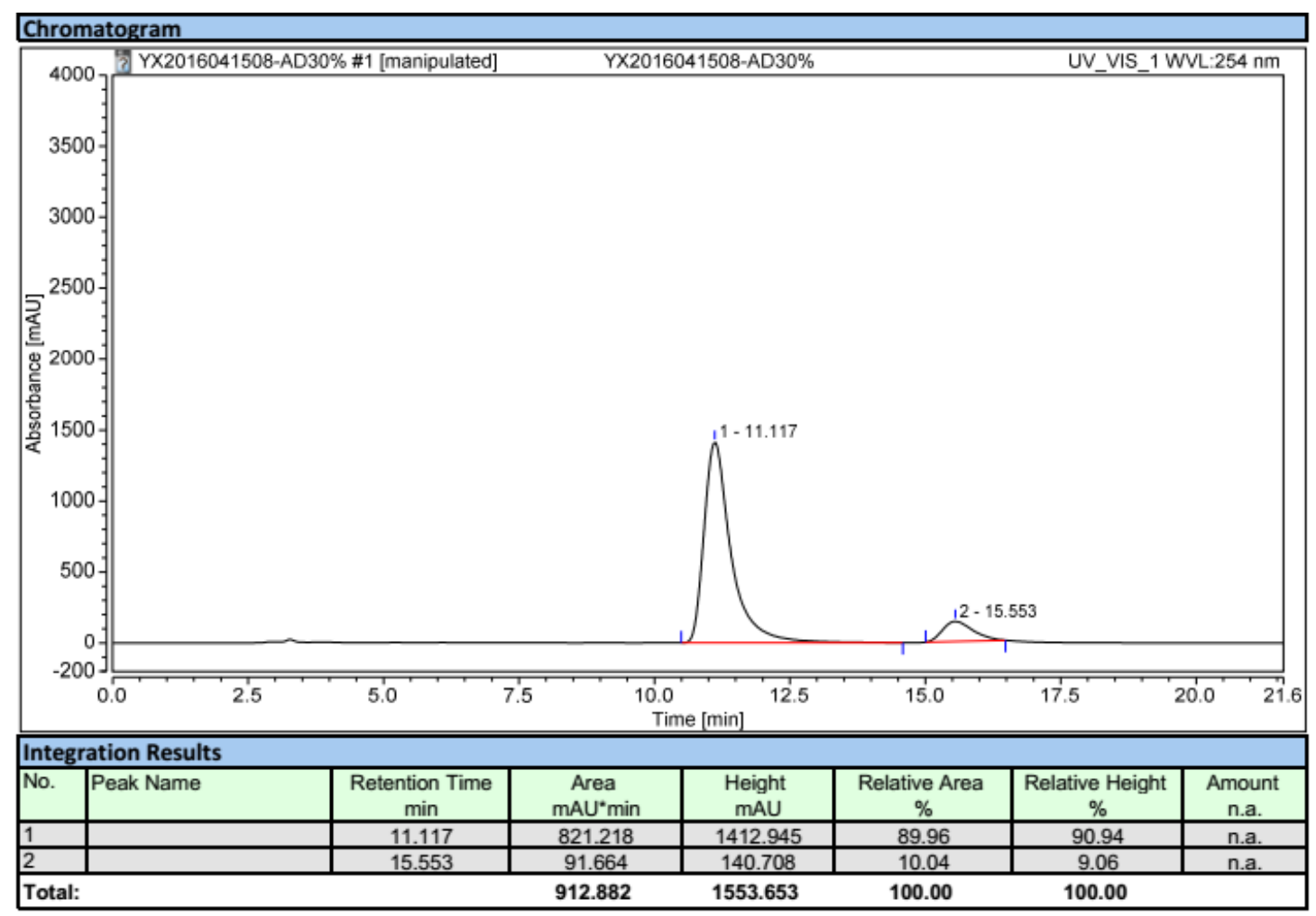




\section{3ae}

Racemic product:

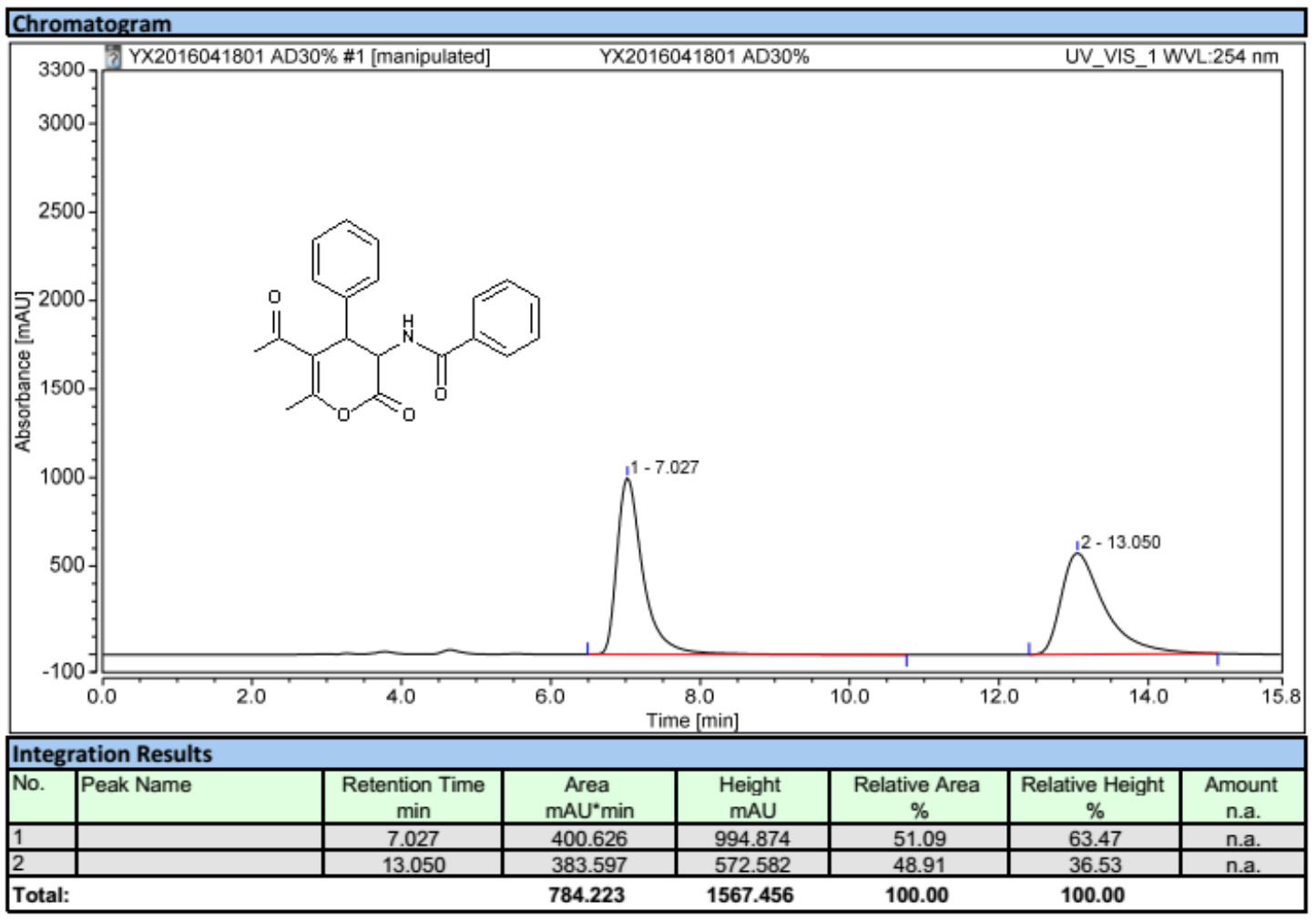

Enantioselective product:

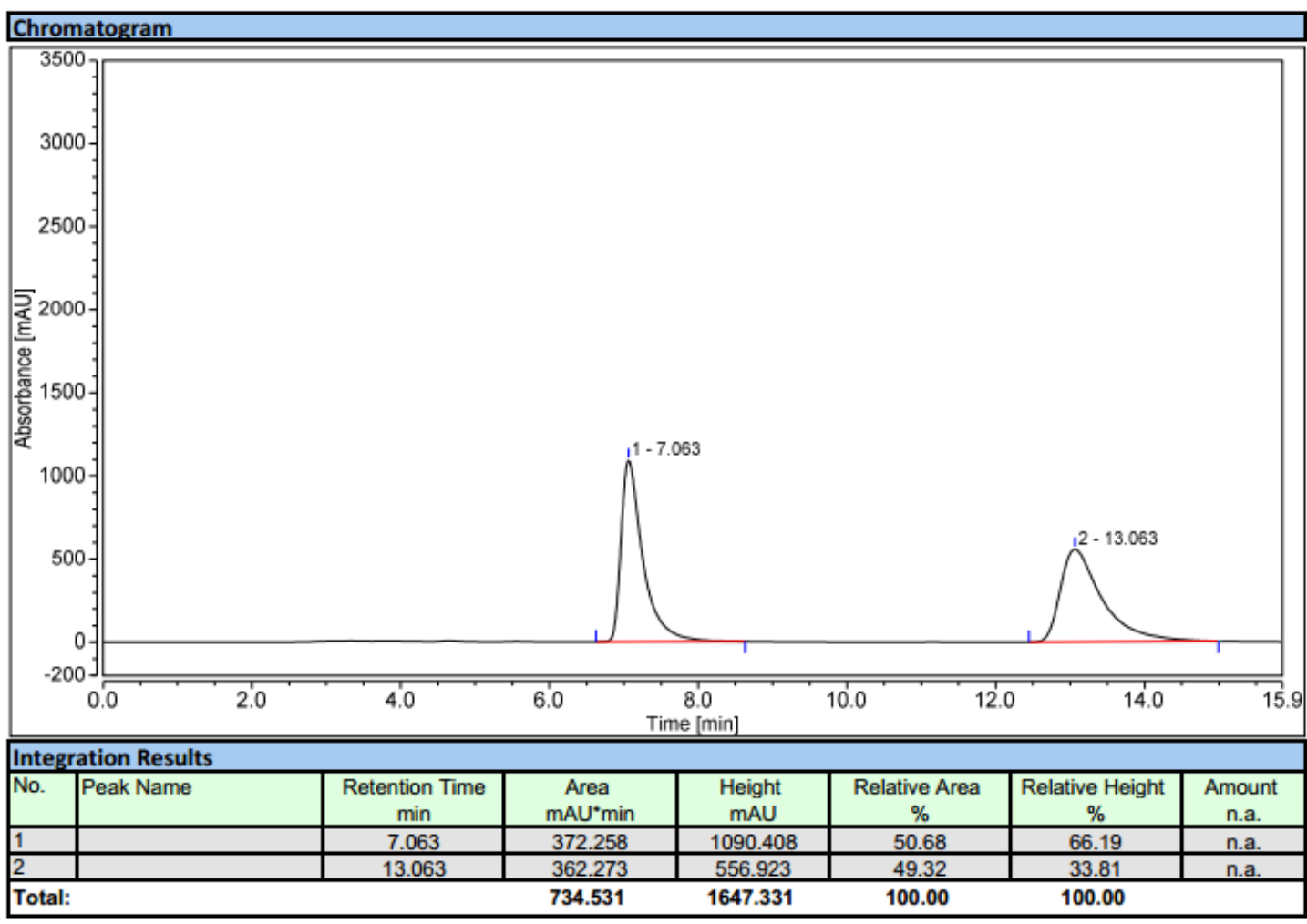




\section{Compound 5}

Racemic product:

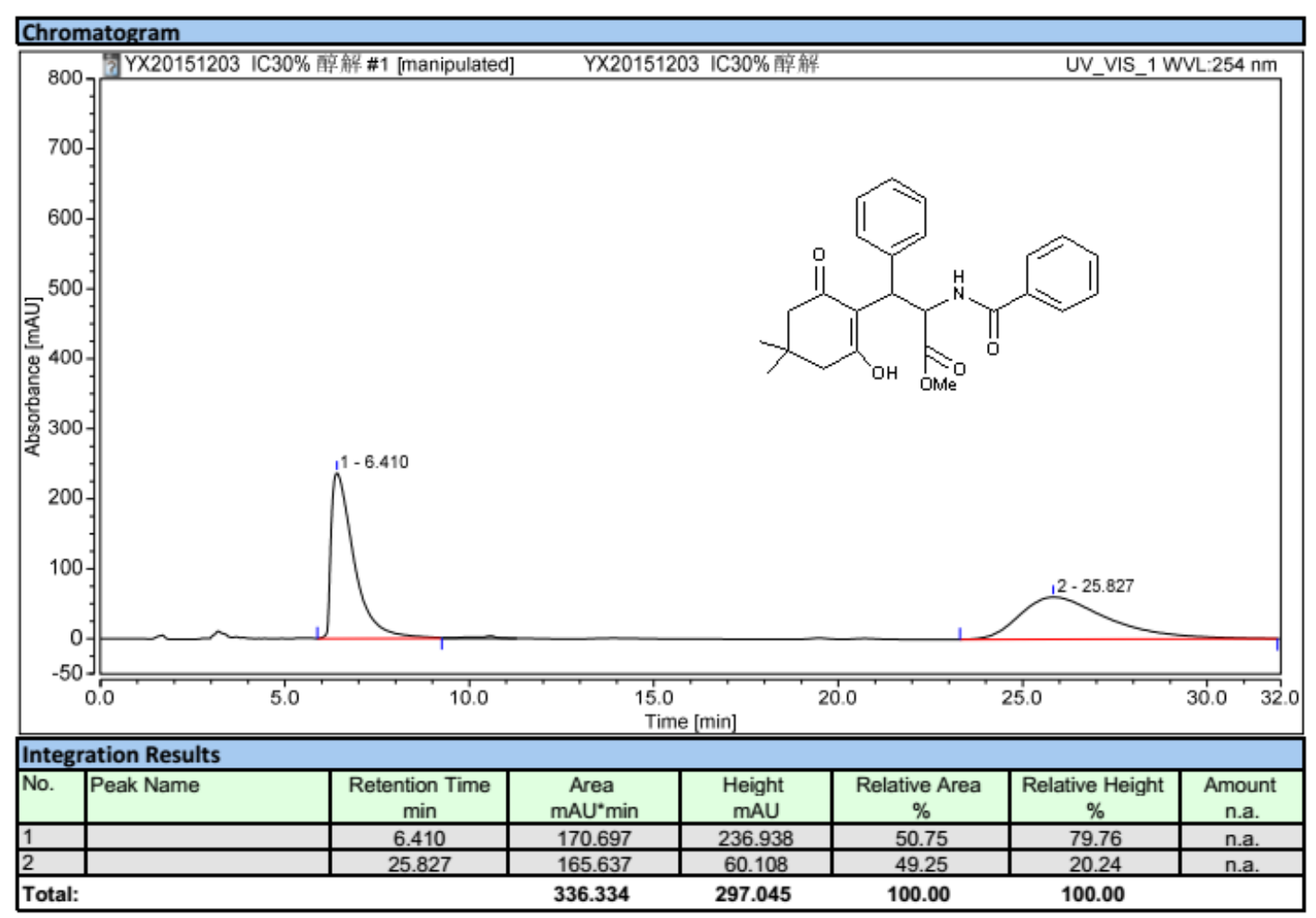

Enantioselective product:

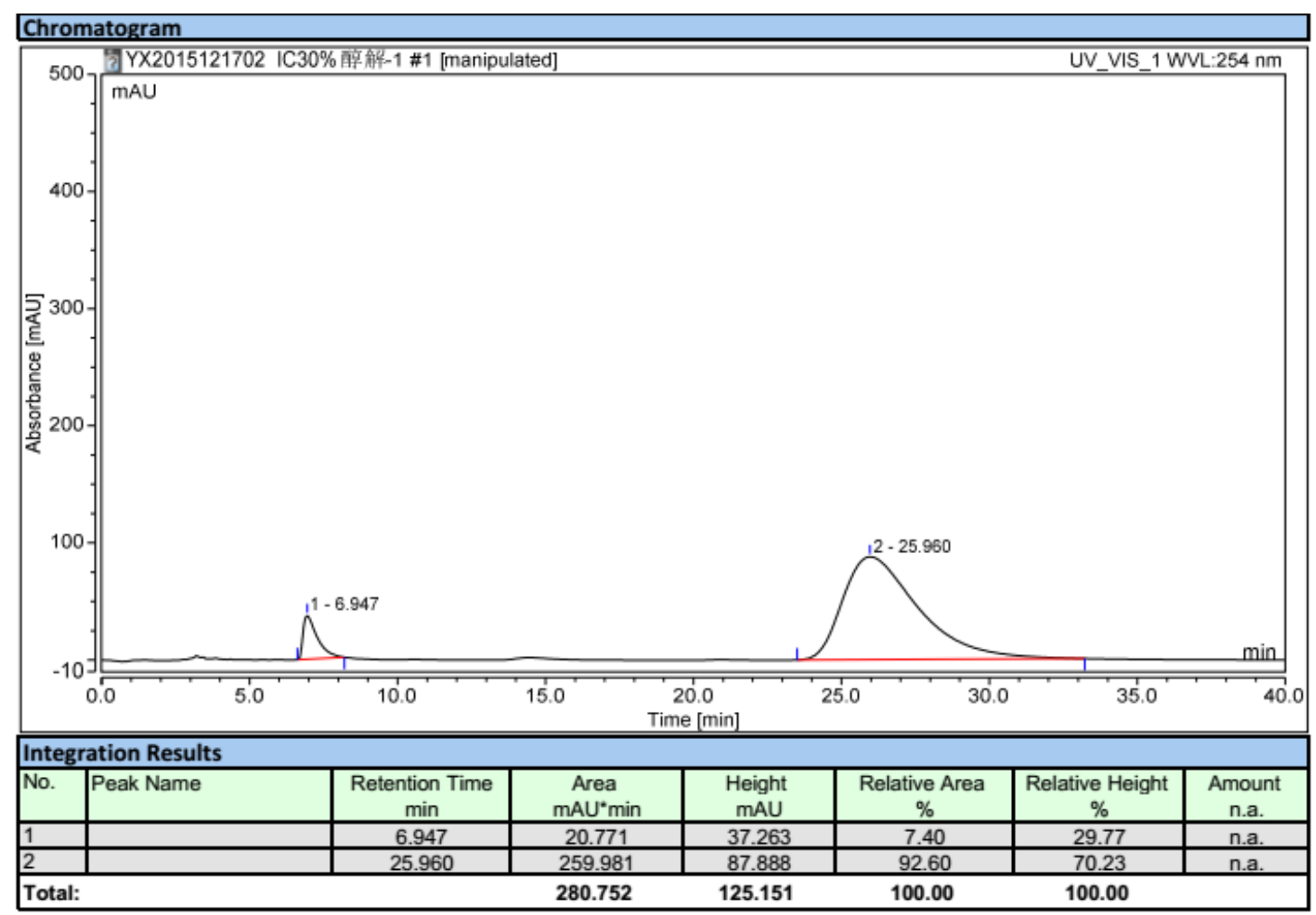




\section{X-ray single crystal data for 3ka}<smiles>COc1ccccc1[C@H]1C2=C(CC(C)(C)CC2=O)OC(=O)[C@H]1NC(=O)c1ccccc1</smiles>

$(3 R, 4 R)-3 \mathrm{ka}$

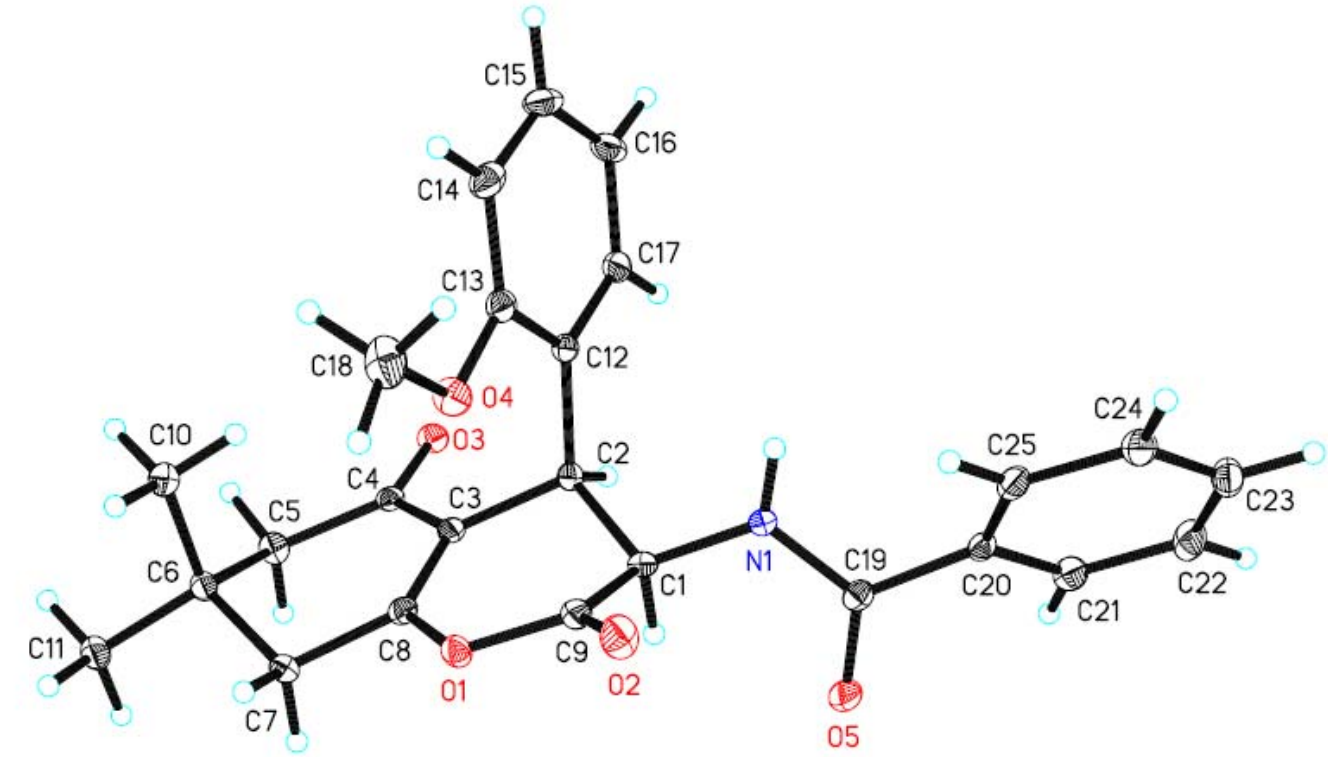

The thermal ellipsoid was drawn at the $30 \%$ probability level.

Empirical formula

Formula weight

Temperature

Wavelength

Crystal system

Space group

Unit cell dimensions

Volume

Z

Density (calculated)

Absorption coefficient

$\mathrm{F}(000)$

Crystal size

Theta range for data collection
$\mathrm{C}_{25} \mathrm{H}_{25} \mathrm{~N} \mathrm{O}_{5}$

419.46

$130 \mathrm{~K}$

$1.54178 \AA$

Monoclinic

P 1211

$\mathrm{a}=9.46700(10) \AA$

$\alpha=90^{\circ}$.

$\mathrm{b}=9.02990(10) \AA$

$\beta=110.7560(10)^{\circ}$.

$c=13.0503(2) \AA$

$\gamma=90^{\circ}$.
2

$1.335 \mathrm{Mg} / \mathrm{m}^{3}$

$0.759 \mathrm{~mm}^{-1}$

444

$0.2 \times 0.15 \times 0.05 \mathrm{~mm}^{3}$

3.622 to $69.675^{\circ}$. 
Index ranges

Reflections collected

Independent reflections

Completeness to theta $=67.679^{\circ}$

Absorption correction

Max. and min. transmission

Refinement method

Data / restraints / parameters

Goodness-of-fit on $\mathrm{F}^{2}$

Final $\mathrm{R}$ indices [I>2sigma(I)]

$\mathrm{R}$ indices (all data)

Absolute structure parameter

Extinction coefficient

Largest diff. peak and hole $-11<=\mathrm{h}<=11,-10<=\mathrm{k}<=8,-15<=\mathrm{l}<=15$

7845

$2832[\mathrm{R}(\mathrm{int})=0.0465]$

$99.2 \%$

Semi-empirical from equivalents

1.0000 and 0.1631

Full-matrix least-squares on $\mathrm{F}^{2}$

2832 / 1 / 283

1.035

$\mathrm{R} 1=0.0328, \mathrm{wR} 2=0.0788$

$\mathrm{R} 1=0.0337, \mathrm{wR} 2=0.0796$

$0.18(19)$

$\mathrm{n} / \mathrm{a}$

0.167 and -0.186 e. $\AA^{-3}$ 